\title{
Modified Lennard-Jones Potentials with a Reduced Temperature-Correction Parameter for Calculating Thermodynamic and Transport Properties: Noble Gases and Their Mixtures (He, Ne, Ar, Kr, and $\mathrm{Xe}$ )
}

\author{
Seung-Kyo Oh \\ Pharmaceutics and Biotechnology Department, Konyang University, 121 University Road, Nonsan, \\ Chungnam 320-711, Republic of Korea
}

Correspondence should be addressed to Seung-Kyo Oh; sunkist@konyang.ac.kr

Received 7 November 2012; Accepted 28 January 2013

Academic Editor: Bill Acree

Copyright (C) 2013 Seung-Kyo Oh. This is an open access article distributed under the Creative Commons Attribution License, which permits unrestricted use, distribution, and reproduction in any medium, provided the original work is properly cited.

\begin{abstract}
The three-parameter Lennard-Jones (12-6) potential function is proposed to calculate thermodynamic property (second virial coefficient) and transport properties (viscosity, thermal conductivity, and diffusion coefficient) of noble gases ( $\mathrm{He}, \mathrm{Ne}, \mathrm{Ar}, \mathrm{Kr}$, and $\mathrm{Xe}$ ) and their mixtures at low density. Empirical modification is made by introducing a reduced temperature-correction parameter $\tau$ to the Lennard-Jones potential function for this purpose. Potential parameters $(\sigma, \varepsilon$, and $\tau)$ are determined individually for each species when the second virial coefficient and viscosity data are fitted together within the experimental uncertainties. Calculated thermodynamic and transport properties are compared with experimental data by using a single set of parameters. The present study yields parameter sets that have more physical significance than those of second virial coefficient methods and is more discriminative than the existing transport property methods in most cases of pure gases and of gas mixtures. In particular, the proposed model is proved with better results than those of the two-parameter Lennard-Jones (12-6) potential, Kihara Potential with group contribution concepts, and other existing methods.
\end{abstract}

\section{Introduction}

Accurate representation of thermodynamic and transport properties is essential to process engineers to design and optimize equipment and chemical processes. Second virial coefficient is an important quantity which is useful in calculating vessel size from volumetric data, heating requirements from calorimetric data, and stage requirements from phase equilibrium data. Transport properties such as viscosity, thermal conductivity, and diffusion coefficient are critically important parameters in many engineering applications: for the determination of pipeline, heatexchanger and separation equipment size, mass transfer efficiency of reservoir of oils, and the power required to pump fluid [1].

The intermolecular forces are of great importance to scientists in a wide field of disciplines as information of these interactions provides the progress of collisions between molecules and determines the bulk properties of substances. Approximation of thermodynamic and transport properties from statistical mechanics requires a realistic intermolecular potential [2]. The theoretical basis in statistical mechanics for the virial equation is one of its attractions. The viral equation truncated after the second term is a popular tool to calculate accurate thermodynamic properties at low or moderate densities. A number of investigators have emphasized the determination of second virial coefficient through experiments and correlations. When Chapman-Enskog gas kinetic theory [3] allows the prediction of transport properties, the potential energy of molecular interactions is known as a function of intermolecular separation and orientation. A description of the spherically symmetric potential as a function of intermolecular separation, averaged over 
all molecular orientations, suffices to calculate dilute gas viscosities, thermal conductivities, and diffusion coefficients of monoatomic gases.

A realistic intermolecular potential allows the calculation of thermodynamic and transport properties. A lot of studies have focused on individual properties like second virial coefficient or viscosity for the determination of intermolecular potential parameters [2]. Potential parameters of any given model that give the best fit for thermodynamic and transport properties (e.g., second virial coefficient, viscosity, thermal conductivity, and diffusion coefficient) are generally different. Therefore, for a simple model such as Lennard-Jones potential, there is one specific set of potential parameters suitable for each property, producing significantly different results [4].

Several investigators [2, 5-7] have used statistical mechanics and kinetic theory of gases to represent thermodynamic and transport properties with a single set of molecular parameters, namely, those appearing in an intermolecular potential function. The Lennard-Jones (12-6) potential has been widely used for the representation of thermodynamic and transport properties of normal fluids. In one particularly interesting study of Tee et al. [6], a single set of molecular parameters was evaluated from the Lennard-Jones (12-6) potential for each species; in this procedure, viscosity data for each substance was fitted first by least-squares analysis, second virial coefficient was fitted next, and the data on second virial coefficient and viscosity were statistically analyzed simultaneously to develop corresponding states correlations with a single set of potential parameters for each substance. They concluded that when second virial coefficient and viscosity data were fitted together, their sets of molecular parameters give the best overall fit to the data for each species and tend to be least affected by experimental errors; beside that their results are quite comparable to those determined individually from viscosity. Potential parameters obtained in this manner were proved to be successful in predicting second virial coefficients and dilute gas viscosities for molecules ranging in shape from spherical to chains as long as n-heptane with good result. Hence, the Lennard-Jones potential is still attractive for its simplicity and capability of predicting noble gas properties if its weak point is compensated for and its accuracy is improved.

The objective of this study is to represent thermodynamic property (second virial coefficient) and transport properties (viscosity, thermal conductivity, and diffusion coefficient) of noble gases ( $\mathrm{He}, \mathrm{Ne}, \mathrm{Ar}, \mathrm{Kr}$, and $\mathrm{Xe}$ ) and their binary mixtures at low density using a single set of modified Lennard-Jones (12-6) potential parameters. For this purpose, a temperature-correction parameter was introduced to the reduced temperature $T^{*}$ in the Lennard-Jones (12-6) potential function. A set of potential parameters was determined when the second virial coefficient and viscosity data are fitted simultaneously within their experimental errors, separately for each noble gas; parameters obtained in this manner were used in all subsequent calculations of properties such as thermal conductivity and diffusion coefficient, in which data were not supplied to parameter estimations, and in mixture property computations. Validity of the modified LennardJones (12-6) potential with a reduced temperature-correction parameter was tested with good results in comparison with other existing methods.

\section{Theory}

In this paper, special focus was placed on the Lennard-Jones (12-6) intermolecular potential for the computations of noble gas properties, even though more accurate potentials exist. A form of this potential was first established by Lennard-Jones [8] and is a mathematically simple model that approximates the interaction between a pair of neutral atoms or molecules. The most common expression of the Lennard-Jones (12-6) potential has the form

$$
U(r)=4 \varepsilon\left[\left(\frac{\sigma}{r}\right)^{12}-\left(\frac{\sigma}{r}\right)^{6}\right],
$$

where $U(r)$ is the intermolecular potential energy as a function of the separation distance between a pair of molecules, $\varepsilon$ is the depth of the potential well in Joule, and $\sigma$ is the finite distance in angstrom $\AA$ at which the interparticle potential becomes zero.

These potential parameters can be fitted by least-squares analysis and lead to provide accurate calculations of dilute gas thermodynamic property (second virial coefficient) and transport properties (viscosity, thermal conductivity, and diffusion coefficient) of noble gases, as summarized below.

2.1. Second Virial Coefficient. From statistical mechanics, the relations between second virial coefficient and intermolecular potential functions were theoretically derived; in particular, explicit expression of the second virial coefficient for the Lennard-Jones (12-6) potential was rigorously derived by Hirschfelder et al. [4] for a computational use:

$$
B(T)=-\frac{2}{3} \pi N_{A} \sigma^{3} \sum_{n=0}^{\infty} \frac{2^{n+1 / 2}}{4 n !} \Gamma\left(\frac{2 n-1}{4}\right) T^{*-(2 n+1) / 4}
$$

in which $N_{A}$ is the Avogadro's constant $6.022 \cdot 10^{23} \mathrm{~mol}^{-1}, T^{*}$ is the reduced (dimensionless) temperature $k_{B} T / \varepsilon$, and $\kappa_{B}$ is the Boltzmann constant $1.3806488 \cdot 10^{-23} \mathrm{JK}^{-1}$. However, Kojima [9] observed that it is quite effective for calculating virial coefficients from the Stockmayer intermolecular potential model with an aid of introducing a reduced temperaturecorrection parameter of $\tau$ as $(T-\tau)$ instead of using temperature $T$. This temperature-correction parameter was also proved in developing a new virial equation of state by Ichikura et al. [10].

In the present study, our observations on the accurate approximation not only of thermodynamic property (second virial coefficient), but also of transport properties (viscosities, thermal conductivities, and diffusion coefficients) in the dilute gaseous phase of noble gases were made by introducing temperature-correction parameter $\tau$ to the reduced temperature $T^{*}$ shown in the Lennard-Jones (12-6) potential function:

$$
T^{*}=\frac{\kappa_{B}(T-\tau)}{\varepsilon} .
$$


Then the second virial coefficient can be calculated from (2) and (3) when three potential parameters $(\sigma, \varepsilon$, and $\tau$ ) of the modified Lennard-Jones (12-6) potential are fitted together to second virial coefficient and viscosity data separately for each substance.

For interaction of different molecular species, the combining rules are used for the molecular distance, energy, and reduced temperature-correction parameter for mixture computations:

$$
\begin{aligned}
& \sigma_{12}=\frac{\sigma_{1}+\sigma_{2}}{2}, \\
& \varepsilon_{12}=\left(\varepsilon_{1} \varepsilon_{2}\right)^{1 / 2}, \\
& \tau_{12}=\frac{\tau_{1}+\tau_{2}}{2} .
\end{aligned}
$$

For spherical molecules, these equations are of high accuracy for the prediction of second cross-virial coefficient $B_{12}$ required in (2) and other mixture properties discussed later.

2.2. Viscosity. Transport properties (viscosity, thermal conductivity, and diffusion coefficient) at low density can be calculated by using Chapman-Enskog kinetic theory [3], which has been applied rigorously to monatomic gases in a number of studies [11]. The dilute gas viscosity can be well presented by the Chapman-Enskog approximation derived from the kinetic theory:

$$
\eta=26.693 \frac{\sqrt{M T}}{\sigma^{2} \Omega^{(2,2)^{*}}\left(T^{*}\right)},
$$

where $M$ is the molecular weight $\left(\mathrm{gram} \mathrm{mol}{ }^{-1}\right.$ ), $T$ is the absolute temperature in Kelvin, and the viscosity $\eta$ is in micropoise $\mu \mathrm{P}$. For mixture viscosity calculations, the formula proposed by Hirschfelder et al. [4] was used in this study, in which the interaction quantity $\eta_{12}$ must be determined to employ this method in advance:

$$
\eta_{12}=26.693 \frac{\sqrt{2 M_{1} M_{2} T /\left(M_{1}+M_{2}\right)}}{\sigma^{2}{ }_{12} \Omega^{(2,2)^{*}}\left(T_{12}^{*}\right)}
$$

in which $M_{1}$ and $M_{2}$ are the molecular weights of the components 1 and 2, respectively, and $T^{*}{ }_{12}$ is the modified reduced temperature for mixture calculations, $k_{B}\left(T-\tau_{12}\right) / \varepsilon_{12}$. The composition dependence of viscosity on the binary gas mixture is defined as follows:

$$
\begin{gathered}
\frac{1}{\eta_{\text {mix }}}=\frac{X_{\eta}+Y_{\eta}}{1+Z_{\eta}}, \\
X_{\eta}=\frac{y_{1}^{2}}{\eta_{1}}+\frac{2 y_{1} y_{2}}{\eta_{12}}+\frac{y_{2}^{2}}{\eta_{2}}, \\
Y_{\eta}=\frac{3}{5} A^{*}{ }_{12}\left[\frac{y_{1}^{2}}{\eta_{1}}\left(\frac{M_{1}}{M_{2}}\right)+\frac{2 y_{1} y_{2}}{\eta_{12}}\left[\frac{\left(M_{1}+M_{2}\right)^{2}}{4 M_{1} M_{2}}\right]\right. \\
\left.\times\left(\frac{\eta_{12}^{2}}{\eta_{1} \eta_{2}}\right)+\frac{y_{2}^{2}}{\eta_{2}}\left(\frac{M_{2}}{M_{1}}\right)\right],
\end{gathered}
$$

$$
\begin{aligned}
& Z_{\eta}=\frac{3}{5} A^{*}{ }_{12}[ y_{1}{ }^{2}\left(\frac{M_{1}}{M_{2}}\right)+2 y_{1} y_{2} \\
& \times\left[\frac{\left(M_{1}+M_{2}\right)^{2}}{4 M_{1} M_{2}}\left(\frac{\eta_{12}}{\eta_{1}}+\frac{\eta_{12}}{\eta_{2}}-1\right)\right] \\
&+\left.y_{2}{ }^{2}\left(\frac{M_{2}}{M_{1}}\right)\right], \\
& A^{*}{ }_{12}=\left(\frac{\Omega^{(2,2)^{*}}}{\Omega^{(1,1)^{*}}}\right),
\end{aligned}
$$

where $\eta_{\text {mix }}$ is the mixture viscosity, and $y_{i}, M_{i}$, and $\eta_{i}$ are the mole fractions, the molecular weights, and the viscosities at the mixture temperature of the pure components $i(i=$ $1,2)$. And the quantities $\Omega^{(2,2)^{*}}$ and $\Omega^{(1,1)^{*}}$ are the collision integrals for viscosity and diffusion coefficient, respectively, and are defined as a function of the reduced temperature $T^{*}$ which depends on the intermolecular potential selected. Neufeld et al. [12] proposed analytical approximations to transport collision integrals for the Lennard-Jones (12-6) potential in the range $0.3<T^{*}<100$, being convenient for easy computer application:

$$
\begin{aligned}
\Omega^{(2,2)^{*}}\left(T^{*}\right)= & \frac{1.16145}{T^{* 0.14874}}+\frac{0.52487}{\exp \left(0.77320 T^{*}\right)} \\
& +\frac{2.16178}{\exp \left(2.43787 T^{*}\right)}, \\
\Omega^{(1,1)^{*}}\left(T^{*}\right)= & \frac{1.06036}{T^{* 0.15610}}+\frac{0.1930}{\exp \left(0.47635 T^{*}\right)} \\
& +\frac{1.03587}{\exp \left(1.52996 T^{*}\right)}+\frac{1.76474}{\exp \left(3.89411 T^{*}\right)} .
\end{aligned}
$$

2.3. Thermal Conductivity. Since the Chapman-Enskog gas kinetic theory uses a common basis for the evaluation of viscosity and thermal conductivity, the statistical expression for the thermal conductivity involves the same collision integral as does the viscosity. For a pure monoatomic gas at low density, which has no rotational or vibrational degrees of freedom, thermal conductivity was calculated through a rigorous analysis by Brokaw [13]:

$$
\lambda=0.0026693 \frac{\sqrt{T / M}}{\sigma^{2} \Omega^{(2,2)^{*}}\left(T^{*}\right)}=\frac{15}{4}\left(\frac{R}{M}\right) \eta,
$$

where the thermal conductivity $\lambda$ is microwatts per meter per degree Kelvin in $\mathrm{mW} \mathrm{m}^{-1} \mathrm{~K}^{-1}$ and $R$ is the universal gas constant $83.14 \mathrm{~cm}^{3}$ bar mol${ }^{-1} \mathrm{k}^{-1}$. Thus, the thermal conductivity of the noble gas can be calculated from the estimated potential parameters or from experimental viscosity data which are generally available for the molecule. In the present work, pure viscosity value obtained from (7) was used for the calculation of thermal conductivity of pure substance. It thus implies that a check on the thermal conductivity serves as a cross-check between the viscosity and thermal conductivity 
TABLE 1: Potential parameters of Lennard-Jones (12-6) Potential with a reduced temperature-correction parameter.

\begin{tabular}{lccc}
\hline Group & $\sigma / \AA ̊$ & $\left(\varepsilon / \kappa_{B}\right) / \mathrm{K}$ & $\tau / \mathrm{K}$ \\
\hline Helium & 2.628 & 5.465 & -0.836 \\
Neon & 2.775 & 36.831 & -2.468 \\
Argon & 3.401 & 116.81 & 5.642 \\
Krypton & 3.601 & 164.56 & 11.41 \\
Xenon & 4.055 & 218.18 & 13.09 \\
\hline
\end{tabular}

data and not necessarily as a check on the potential function chosen [14].

Various prediction methods for estimating mixture thermal conductivity have appeared, one of which is essentially empirical and it is reduced to some form of the Wassiljewa equation [15]:

$$
\lambda_{\text {mix }}=\sum_{i=1}^{n} \frac{y_{i} \lambda_{i}}{\sum_{j=1}^{n} y_{j} A_{i j}},
$$

where $\lambda_{\text {mix }}$ is the mixture thermal conductivity and $A_{i j}$ is a combinational factor. This factor is empirically expressed by Mason and Saxena [16] as

$$
A_{i j}=\frac{1.065}{2 \sqrt{2}} \frac{\left[1+\left(\lambda_{i} / \lambda_{j}\right)^{1 / 2}\left(M_{i} / M_{j}\right)^{1 / 4}\right]^{2}}{\left(1+M_{i} / M_{j}\right)^{1 / 2}}
$$

where $\lambda_{i}$ are the thermal conductivities of pure component determined from (16), which are sufficient to predict mixture thermal conductivity when its measurements are not available in the literature.

2.4. Diffusion Coefficient. The Chapman-Enskog expression for binary diffusion coefficient of dilute gas is presented by Hirschfelder et al. [4]:

$$
D_{12}=0.0026693 T^{3 / 2} \frac{\sqrt{\left(M_{1}+M_{2}\right) / 2 M_{1} M_{2}}}{P \sigma^{2}{ }_{12} \Omega^{(1,1)^{*}}\left(T^{*}{ }_{12}\right)},
$$

where $D_{12}$ is in $\mathrm{cm}^{2} \sec ^{-1}$ and $P$ is in bar. And $\Omega^{(1,1)^{*}}$ is the collision integral for diffusion coefficient of nonpolar Lennard-Jones (12-6) potential given by (15). When molecules 1 and 2 are identical, (19) becomes expression for the self-diffusion coefficient:

$$
D=0.0026693 T^{3 / 2} \frac{\sqrt{1 / M}}{P \sigma^{2} \Omega^{(1,1)^{*}}\left(T^{*}\right)} .
$$

\section{Results and Discussion}

3.1. Pure Noble Gases. As a part of systematic program of our researches, modified Lennard-Jones (12-6) potential function with a reduced temperature-correction parameter was applied to noble gases ( $\mathrm{He}, \mathrm{Ne}, \mathrm{Ar}, \mathrm{Kr}$, and $\mathrm{Xe}$ ) for the computation of thermodynamic (second virial coefficient) and transport properties (viscosity, thermal conductivity, and self-diffusion coefficient) at low density.

Using (2), (3), and (7), three potential parameters $(\sigma, \varepsilon$, and $\tau$ ) were evaluated from the simultaneous regression of second virial coefficient and viscosity data separately for each species. For instance, three parameter values $\left(\sigma_{\mathrm{He}}, \varepsilon_{\mathrm{He}}\right.$, and $\left.\tau_{\mathrm{He}}\right)$ for helium gas were determined from second virial coefficient and viscosity data of pure helium gas. Potential parameters of other noble gases were evaluated in an analogous manner.

A critical review of the literature on second virial coefficient was achieved by Dymond et al. [17]. They provided the recommended values of virial coefficients for each compound fitted to a smoothing function of temperature by the leastsquares criterion. This smoothing function for second virial coefficient is the polynomial of reciprocal temperature with usually three terms. In particular, two different smoothing correlations of helium were given in the temperature ranges between $1.59 \mathrm{~K}$ and $35.1 \mathrm{~K}$ and between $35.1 \mathrm{~K}$ and $1473.15 \mathrm{~K}$. We have used their comprehensive compilation of the second virial coefficient data as our data source. And pure viscosity data required for the potential parameter determinations were all taken from Stephan and Lucas [18], even though other recommended or various sets of data exist. Nonlinear least-squares parameter estimation subroutine based on the Levenberg-Marquardt algorithm supplied by IMSL STAT/library [19] was used in this data regression, in which each data point was weighted by its estimated experimental uncertainty taken from the corresponding Refs. A set of potential parameters individually for each substance can then be estimated when the following objective function is minimized:

$$
\Phi=\sum_{i=1}^{n_{B}}\left(\frac{B_{i, \mathrm{obsd}}-B_{i, \mathrm{calc}}}{\delta_{i, B}}\right)^{2}+\sum_{i=1}^{n_{\eta}}\left(\frac{\eta_{i, \mathrm{obsd}}-\eta_{i, \mathrm{calc}}}{\eta_{i, \mathrm{obsd}} \delta_{i, \eta}}\right)^{2}
$$

in which $\delta_{B}$ is the observed uncertainty of second virial coefficient in $\mathrm{cm}^{3} \mathrm{~mol}^{-1}$ and $\delta_{\eta}$ is of dimensionless fractional uncertainty of viscosity in \%. It is noted that the first term is designated for second virial coefficient and the second for viscosity.

Table 1 summarizes determined parameter values of modified Lennard-Jones (12-6) potential $(\sigma, \varepsilon$, and $\tau$ ) of noble gases ( $\mathrm{He}, \mathrm{Ne}, \mathrm{Ar}, \mathrm{Kr}$, and $\mathrm{Xe}$ ). In Table 2, resulting deviations between observed and regressed second virial coefficient data are given on an RMSD (root-mean-square deviation) basis in $\mathrm{cm}^{3} \mathrm{~mol}^{-1}$, which is defined by

$$
\mathrm{RMSD}=\sqrt{\frac{1}{n_{B}} \sum_{i=1}^{n_{B}}\left(B_{i, \mathrm{obsd}}-B_{i, \mathrm{calc}}\right)^{2}}
$$

Comparisons of the proposed method with other existing methods are shown in Table 2 along with their data sources and observed temperature ranges. The average RMSD between a total of observed and calculated 735 second virial coefficient data of five noble gases by the present method was $3.33 \mathrm{~cm}^{3} \mathrm{~mol}^{-1}$, indicating that the proposed method compares very well with the Dymond's correlations 
TABle 2: Deviations between experimental and calculated second virial coefficients of pure noble gases.

\begin{tabular}{|c|c|c|c|c|c|c|c|c|}
\hline \multirow{2}{*}{ Compound } & \multirow{2}{*}{ Number of data points } & \multicolumn{5}{|c|}{ Average RMSD in $B\left(\mathrm{~cm}^{3} \mathrm{~mol}^{-1}\right)$} & \multirow[b]{2}{*}{$\Delta T(\mathrm{~K})$} & \multirow[b]{2}{*}{ Reference } \\
\hline & & Present study & Dymond et al. & $\mathrm{L}-\mathrm{J}$ & Kihara & Tsonopoulos & & \\
\hline \multicolumn{9}{|c|}{ Regression results } \\
\hline Helium & 304 & 5.44 & $5.07^{\mathrm{a}}$ & 10.9 & 10.8 & 39.1 & $2-1473$ & {$[2]$} \\
\hline Neon & 55 & 0.28 & 0.34 & 0.45 & 1.38 & 1.56 & $44-873$ & {$[2]$} \\
\hline Argon & 105 & 0.40 & 0.53 & 2.43 & 1.73 & 1.88 & $75-773$ & {$[2]$} \\
\hline Krypton & 157 & 2.87 & 3.18 & 5.75 & 3.67 & 6.90 & $107-873$ & {$[2]$} \\
\hline Xenon & 114 & 2.50 & 2.67 & 4.32 & 2.53 & 3.51 & $160-973$ & {$[2]$} \\
\hline Average & 735 & 3.33 & 3.29 & 6.79 & 5.99 & 18.6 & & \\
\hline \multicolumn{9}{|c|}{ Prediction results } \\
\hline Helium & 8 & 4.13 & 1.08 & 10.8 & 4.29 & 14.9 & $343-374$ & {$[27]$} \\
\hline Xenon & 9 & 0.50 & 1.37 & 2.57 & 2.11 & 2.57 & $206-273$ & {$[28]$} \\
\hline Average & 17 & 2.21 & 1.23 & 6.44 & 3.14 & 8.37 & & \\
\hline
\end{tabular}

${ }^{\mathrm{a}}$ Two different smoothing functions were used in the temperature ranges between $1.59 \mathrm{~K}$ and $35.1 \mathrm{~K}$, and between $35.1 \mathrm{~K}$ and $1473.15 \mathrm{~K}$.

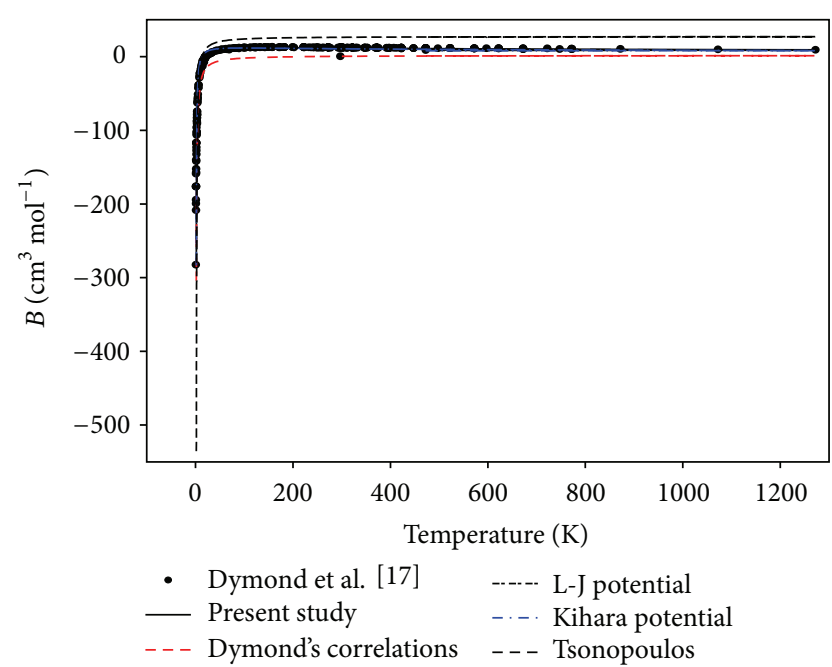

FIGURE 1: Comparison of measured and calculated second virial coefficients for He.

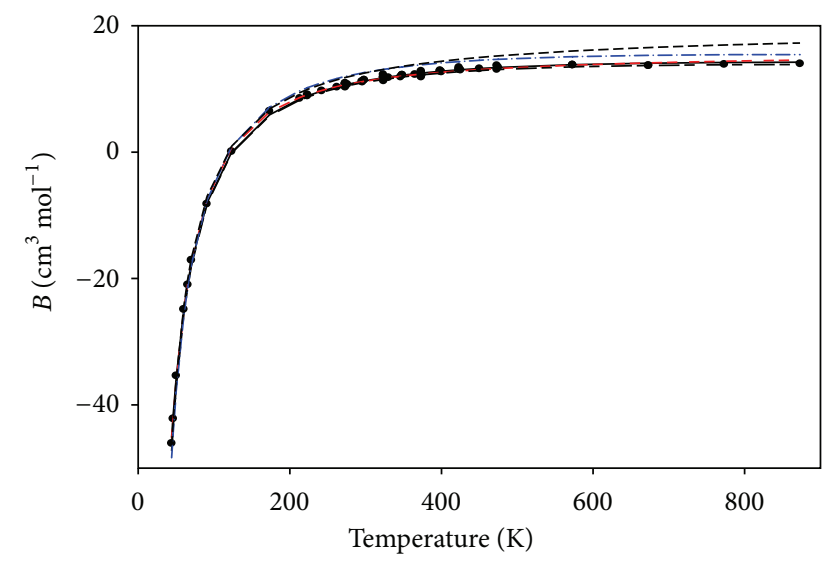
- Dymond et al. [17]
-.--. L-J potential
_ Present study _..- Kihara potential
- - Dymond's correlations _ - - Tsonopoulos

FIGURE 2: Comparison of measured and calculated second virial coefficients for $\mathrm{Ne}$.

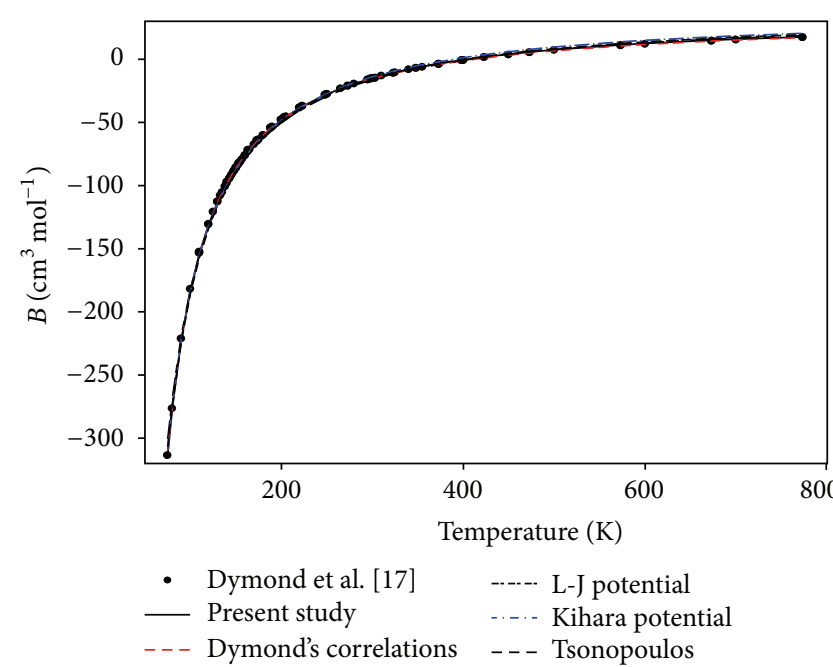

FIGURE 3: Comparison of measured and calculated second virial coefficients for Ar.

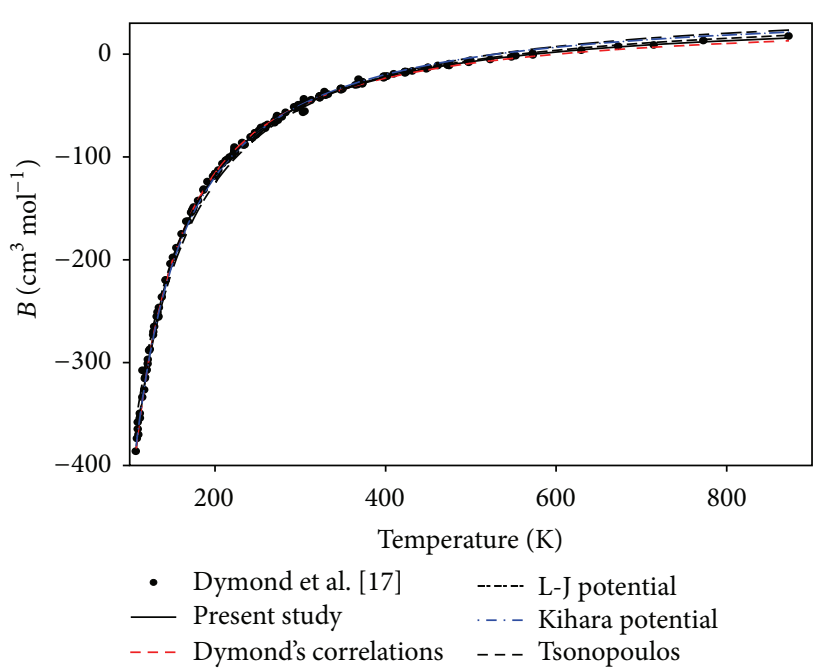

FIGURE 4: Comparison of measured and calculated second virial coefficients for $\mathrm{Kr}$. 


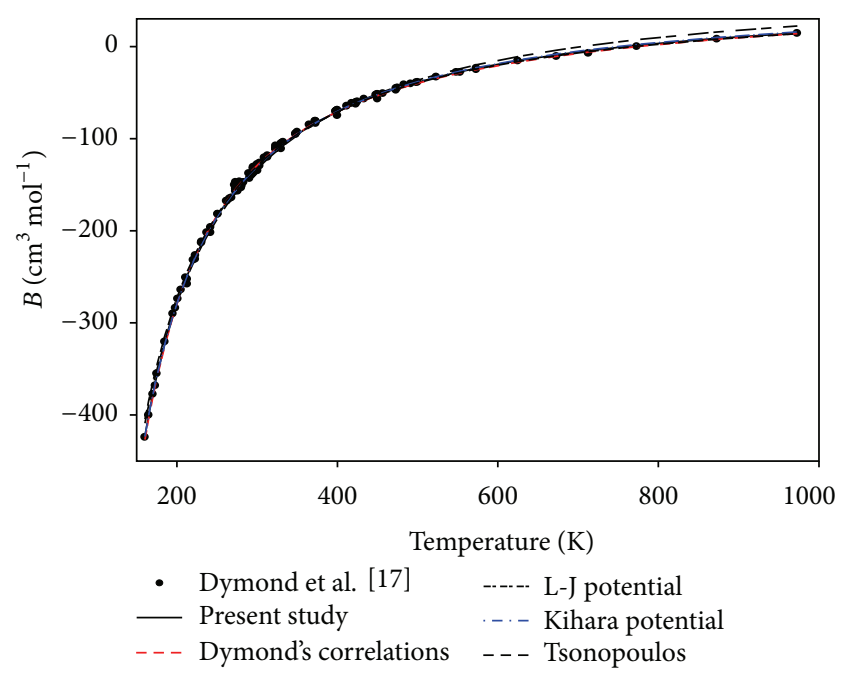

FIGURE 5: Comparison of measured and calculated second virial coefficients for Xe.

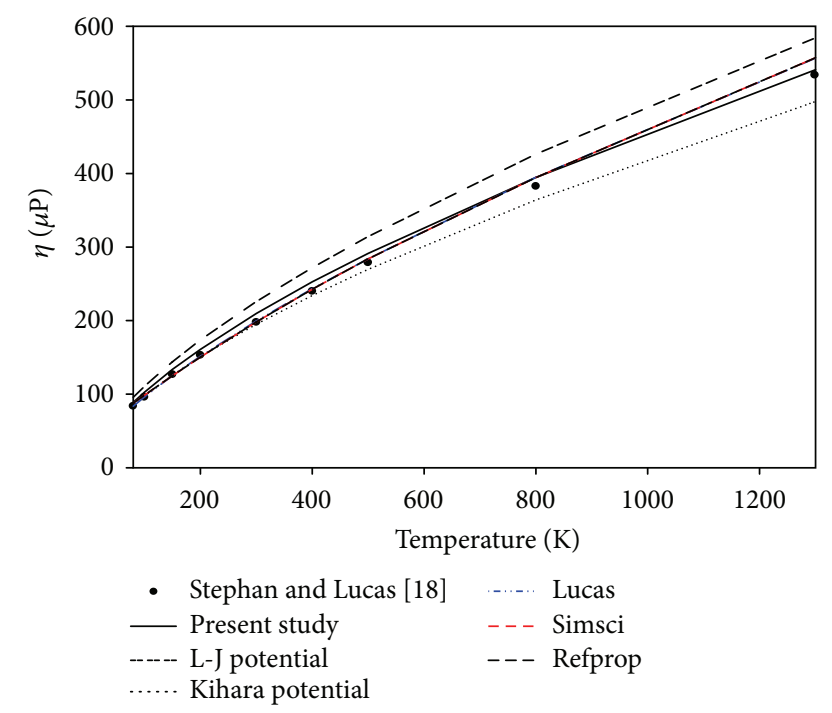

Figure 6: Comparison of measured and calculated viscosities for He.

[17] $3.29 \mathrm{~cm}^{3} \mathrm{~mol}^{-1}$ and yields more accurate results than other existing methods: original two-parameter LennardJones (12-6) potential [4] $6.79 \mathrm{~cm}^{3} \mathrm{~mol}^{-1}$, Kihara potential with group contribution concept $[20] 5.99 \mathrm{~cm}^{3} \mathrm{~mol}^{-1}$, and the corresponding states method of Tsonopoulos [21] $18.6 \mathrm{~cm}^{3} \mathrm{~mol}^{-1}$. A comparison of the measured and calculated second virial coefficients from Dymond et al. [17] for pure noble gases ( $\mathrm{He}, \mathrm{Ne}, \mathrm{Ar}, \mathrm{Kr}$, and $\mathrm{Xe}$ ) is presented in Figures 1, $2,3,4$, and 5 , in order. Also included in Table 2 are prediction results from other second virial coefficient data of helium and xenon available in the literature, showing similar results.

In Table 3, deviations of regression results between observed and calculated viscosity data are presented on an $\mathrm{RMSD}_{\mathrm{r}}$

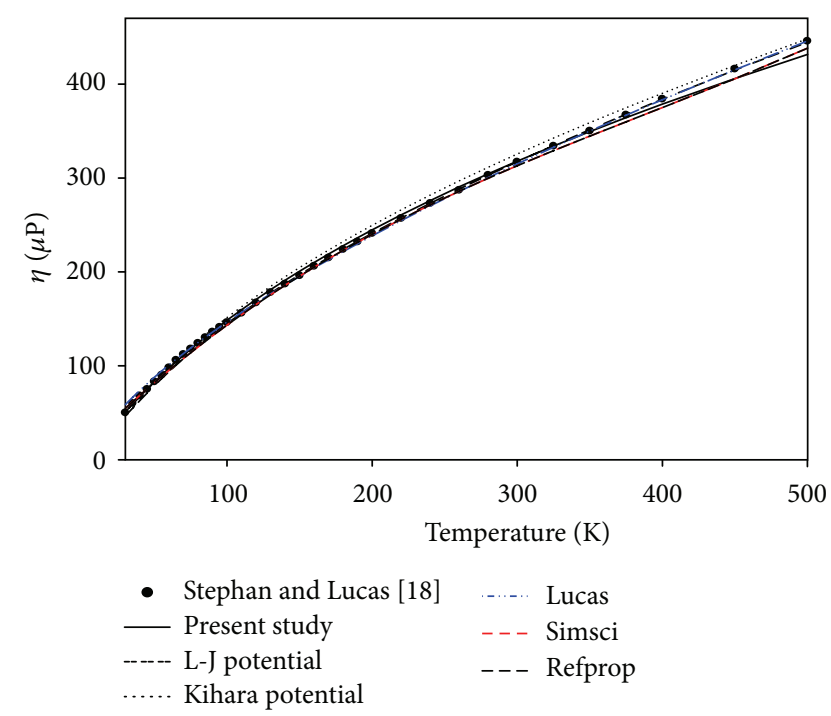

FIgURE 7: Comparison of measured and calculated viscosities for $\mathrm{Ne}$.

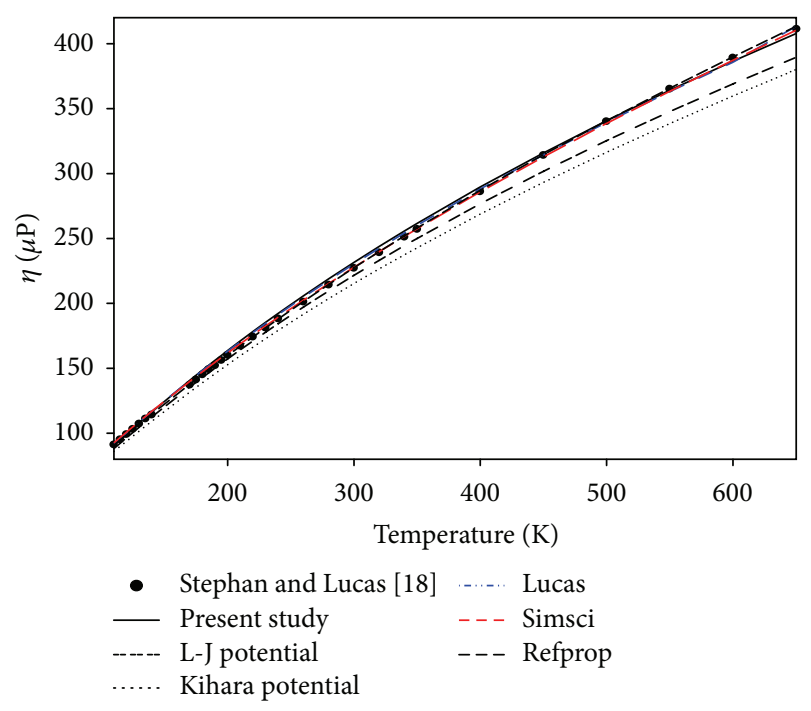

FIGURE 8: Comparison of measured and calculated viscosities for Ar.

(root-mean-square deviation, relative) basis in \%, which is defined by

$$
\mathrm{RMSD}_{\mathrm{r}}=\sqrt{\frac{1}{n_{\eta}} \sum_{i=1}^{n_{\eta}}\left(\frac{\left(\eta_{i, \mathrm{obsd}}-\eta_{i, \mathrm{calc}}\right)}{\eta_{i, \mathrm{obsd}}}\right)^{2}} .
$$

The average $\mathrm{RMSD}_{\mathrm{r}}$ value between a total of observed and regressed 117 viscosity data for five noble gases was found to be $1.90 \%$, indicating that the present work is quite comparable to the Refprop Database correlations [22] 1.78\% and is in better agreement with experimental data than any other existing methods: original Lennard-Jones (12-6) potential $6.60 \%$, Kihara potential with group contribution concept $5.74 \%$, the corresponding states method of Lucas [23] 2.25\%, and the Simsci Database correlations [24] 2.08\%. 
TABLE 3: Deviations between experimental and calculated viscosities of pure noble gases.

\begin{tabular}{|c|c|c|c|c|c|c|c|c|c|}
\hline \multirow{2}{*}{ Compound } & \multirow{2}{*}{ Number of data points } & \multicolumn{6}{|c|}{ Average $\mathrm{RMSD}_{\mathrm{r}}$ in $\eta(\%)$} & \multirow[b]{2}{*}{$\Delta T(\mathrm{~K})$} & \multirow[b]{2}{*}{ Reference } \\
\hline & & Present study & L-J & Kihara & Lucas & Simsci & Refprop & & \\
\hline \multicolumn{10}{|c|}{ Regression results } \\
\hline Helium & 9 & 5.27 & 18.3 & 13.2 & 3.23 & 2.05 & 2.83 & $80-1300$ & {$[24]$} \\
\hline Neon & 36 & 1.50 & 1.86 & 3.76 & 2.80 & 4.29 & 2.48 & $30-500$ & {$[24]$} \\
\hline Argon & 30 & 1.84 & 2.72 & 5.33 & 1.73 & 1.32 & 0.29 & $10-650$ & {$[24]$} \\
\hline Krypton & 19 & 2.87 & 12.2 & 8.56 & 1.23 & 0.91 & 1.85 & $150-600$ & {$[24]$} \\
\hline Xenon & 23 & 0.49 & 9.88 & 4.10 & 2.50 & 0.57 & 2.13 & $300-430$ & {$[24]$} \\
\hline Average & $117^{* *}$ & 1.90 & 6.60 & 5.74 & 2.25 & 2.08 & 1.78 & & \\
\hline \multicolumn{10}{|c|}{ Prediction results } \\
\hline \multirow{33}{*}{ Helium } & 22 & 4.11 & 8.33 & 2.99 & 4.95 & 0.98 & 3.94 & $50-973$ & {$[26]$} \\
\hline & 31 & 4.62 & 9.03 & 2.96 & 3.92 & 0.60 & 10.0 & $20-1000$ & [29] \\
\hline & 1 & 4.44 & 8.82 & 0.76 & 2.87 & 0.64 & 1.32 & 300 & [30] \\
\hline & 8 & 2.34 & 6.52 & 4.03 & 6.74 & 0.91 & 0.84 & $298-1010$ & {$[31]$} \\
\hline & 1 & 4.83 & 9.93 & 0.14 & 2.26 & 0.06 & 0.75 & 303 & {$[32]$} \\
\hline & 13 & 3.15 & 7.32 & 4.24 & 6.36 & 1.31 & 1.43 & $198-473$ & [33] \\
\hline & 8 & 2.90 & 6.86 & 4.17 & 6.42 & 0.40 & 0.53 & 298-973 & [34] \\
\hline & 8 & 2.71 & 6.65 & 4.27 & 6.54 & 0.23 & 0.42 & $298-973$ & [35] \\
\hline & 6 & 2.98 & 6.87 & 4.37 & 6.55 & 0.36 & 0.57 & $298-973$ & [36] \\
\hline & 9 & 3.26 & 7.90 & 2.47 & 4.72 & 0.33 & 0.49 & $298-678$ & [37] \\
\hline & 9 & 2.51 & 6.68 & 3.83 & 6.17 & 0.26 & 0.36 & $298-778$ & [38] \\
\hline & 5 & 4.05 & 8.85 & 1.32 & 3.48 & 0.09 & 0.41 & $298-473$ & [39] \\
\hline & 1 & 2.91 & 7.66 & 2.66 & 5.3 & 3.68 & 3.46 & 767 & [40-42] \\
\hline & 6 & 4.24 & 8.91 & 1.37 & 3.54 & 0.35 & 0.32 & $298-512$ & {$[43]$} \\
\hline & 1 & 5.28 & 10.2 & 0.08 & 2.03 & 0.09 & 0.62 & 292 & {$[44]$} \\
\hline & 1 & 5.72 & 10.5 & 0.77 & 1.34 & 0.76 & 0.03 & 291 & {$[45,46]$} \\
\hline & 2 & 6.29 & 11.1 & 1.40 & 0.98 & 1.39 & 0.76 & 291 & [47] \\
\hline & 2 & 5.06 & 10.1 & 12.7 & 2.12 & 0.13 & 0.60 & $293-303$ & [48] \\
\hline & 4 & 5.48 & 10.4 & 0.95 & 1.82 & 0.51 & 0.81 & $237-308$ & [49] \\
\hline & 6 & 5.91 & 10.7 & 1.51 & 0.36 & 1.50 & 2.50 & $72-291$ & {$[50]$} \\
\hline & 10 & 5.01 & 9.61 & 4.31 & 1.77 & 3.08 & 30.8 & $14-293$ & {$[51]$} \\
\hline & 5 & 5.30 & 10.2 & 0.46 & 2.23 & 1.84 & 1.83 & $297-523$ & {$[52]$} \\
\hline & 2 & 5.07 & 10.1 & 0.13 & 2.11 & 0.12 & 0.59 & $293-303$ & [53] \\
\hline & 1 & 4.85 & 9.99 & 0.02 & 2.16 & 0.14 & 0.54 & 303 & {$[54]$} \\
\hline & 10 & 4.94 & 9.75 & 1.01 & 2.86 & 1.40 & 1.38 & $293-523$ & {$[55]$} \\
\hline & 39 & 6.84 & 10.4 & 2.73 & 6.18 & 5.94 & 22.0 & 14-973 & {$[56]$} \\
\hline & 74 & 3.40 & 7.42 & 3.73 & 5.97 & 0.87 & 0.58 & $100-1000$ & {$[57]$} \\
\hline & 72 & 5.77 & 10.3 & 6.75 & 4.09 & 15.7 & 62.2 & $200-1000$ & {$[58]$} \\
\hline & 8 & 2.69 & 6.63 & 4.28 & 6.55 & 0.24 & 0.44 & 298-973 & [59] \\
\hline & 11 & 4.70 & 9.22 & 1.89 & 3.51 & 0.46 & 0.49 & $100-600$ & {$[60]$} \\
\hline & 19 & 5.32 & 9.72 & 3.05 & 4.30 & 0.35 & 1.68 & 80-1000 & {$[61]$} \\
\hline & 7 & 1.32 & 4.25 & 5.50 & 7.98 & 3.10 & 3.45 & $250-1000$ & [62] \\
\hline & 13 & 3.83 & 8.54 & 1.89 & 4.34 & 2.64 & 2.53 & $373-973$ & {$[63]$} \\
\hline Average & $415^{*}$ & 4.49 & 8.76 & 3.75 & 4.82 & 3.97 & 15.1 & & \\
\hline \multirow{6}{*}{ Neon } & 27 & 2.04 & 1.54 & 8.00 & 3.14 & 3.04 & 15.2 & $50-973$ & {$[26]$} \\
\hline & 6 & 1.58 & 1.22 & 7.79 & 2.45 & 1.47 & 2.20 & $100-600$ & [29] \\
\hline & 8 & 1.58 & 0.72 & 8.36 & 3.65 & 0.57 & 27.4 & $298-973$ & {$[64]$} \\
\hline & 13 & 1.83 & 1.04 & 8.60 & 3.54 & 1.04 & 21.2 & $298-973$ & [33] \\
\hline & 8 & 2.07 & 1.24 & 8.91 & 4.21 & 0.92 & 27.1 & $298-973$ & [35] \\
\hline & 6 & 2.22 & 1.37 & 8.97 & 4.30 & 1.09 & 29.8 & 298-973 & {$[36]$} \\
\hline
\end{tabular}


TABle 3: Continued.

\begin{tabular}{|c|c|c|c|c|c|c|c|c|c|}
\hline \multirow{2}{*}{ Compound } & \multirow{2}{*}{ Number of data points } & \multicolumn{6}{|c|}{ Average $\mathrm{RMSD}_{\mathrm{r}}$ in $\eta(\%)$} & \multirow[b]{2}{*}{$\Delta T(\mathrm{~K})$} & \multirow[b]{2}{*}{ Reference } \\
\hline & & Present study & L-J & Kihara & Lucas & Simsci & Refprop & & \\
\hline & 8 & 2.27 & 1.39 & 9.05 & 4.36 & 1.08 & 26.9 & $298-973$ & {$[65]$} \\
\hline & 5 & 1.13 & 0.46 & 7.91 & 1.82 & 1.07 & 2.72 & $298-473$ & {$[39]$} \\
\hline & 7 & 1.80 & 1.00 & 8.66 & 3.64 & 0.91 & 11.3 & $298-778$ & {$[38]$} \\
\hline & 9 & 1.67 & 0.90 & 8.47 & 2.90 & 1.15 & 3.52 & $298-673$ & {$[37]$} \\
\hline & 3 & 1.07 & 2.10 & 5.89 & 0.82 & 0.66 & 0.47 & $298-348$ & {$[66]$} \\
\hline & 3 & 4.46 & 5.53 & 2.74 & 4.21 & 3.99 & 2.91 & $298-348$ & {$[67,68]$} \\
\hline & 3 & 0.49 & 1.50 & 6.45 & 0.29 & 0.13 & 1.07 & $298-348$ & {$[69]$} \\
\hline & 6 & 1.03 & 2.01 & 6.02 & 0.81 & 0.70 & 0.71 & $298-348$ & {$[40-42]$} \\
\hline & 3 & 0.27 & 1.17 & 6.78 & 0.22 & 0.58 & 1.39 & $298-348$ & {$[70]$} \\
\hline & 3 & 0.19 & 1.17 & 6.76 & 0.22 & 0.33 & 1.39 & $298-348$ & {$[71]$} \\
\hline & 3 & 0.75 & 0.75 & 7.36 & 0.97 & 1.00 & 2.11 & $298-348$ & {$[72]$} \\
\hline & 3 & 0.56 & 0.52 & 7.38 & 0.81 & 0.97 & 2.04 & $298-348$ & {$[73]$} \\
\hline & 3 & 0.49 & 0.56 & 7.32 & 0.74 & 0.92 & 1.98 & $298-348$ & {$[74]$} \\
\hline & 3 & 1.09 & 0.89 & 7.56 & 1.30 & 1.28 & 2.38 & $298-348$ & {$[75]$} \\
\hline & 3 & 0.58 & 0.50 & 7.39 & 0.83 & 0.99 & 2.06 & $298-348$ & {$[76]$} \\
\hline & 4 & 0.65 & 0.305 & 7.45 & 0.64 & 1.61 & 1.71 & $237-308$ & [49] \\
\hline & 2 & 0.65 & 0.328 & 7.50 & 0.78 & 1.35 & 1.94 & $293-303$ & {$[53]$} \\
\hline & 5 & 2.20 & 1.986 & 8.31 & 2.24 & 2.10 & 3.38 & $72-291$ & [77] \\
\hline & 5 & 1.08 & 2.994 & 8.15 & 1.92 & 9.59 & 7.60 & $20-293$ & {$[51]$} \\
\hline & 6 & 2.08 & 2.176 & 7.54 & 2.44 & 2.05 & 2.91 & $273-523$ & {$[52]$} \\
\hline & 2 & 0.65 & 0.328 & 7.50 & 0.78 & 1.35 & 1.94 & $293-303$ & [53] \\
\hline & 2 & 1.12 & 2.129 & 5.76 & 1.14 & 0.47 & 0.17 & 291 & [47] \\
\hline & 2 & 0.52 & 0.947 & 6.98 & 0.51 & 0.92 & 1.39 & 291 & {$[45,46]$} \\
\hline & 52 & 0.98 & 1.568 & 7.83 & 1.68 & 6.64 & 13.4 & $20-1000$ & {$[56]$} \\
\hline & 32 & 1.24 & 0.991 & 7.71 & 3.11 & 0.68 & 22.1 & $100-950$ & [57] \\
\hline & 92 & 4.80 & 4.116 & 5.86 & 4.23 & 12.4 & 7.89 & $27-320$ & {$[58]$} \\
\hline & 8 & 2.08 & 1.242 & 8.92 & 4.21 & 0.93 & 27.1 & $298-973$ & [59] \\
\hline & 11 & 1.77 & 2.693 & 5.38 & 1.53 & 1.83 & 1.92 & $100-600$ & {$[60]$} \\
\hline & 19 & 1.35 & 1.439 & 7.15 & 2.68 & 1.10 & 20.4 & $80-1000$ & {$[61]$} \\
\hline & 1 & 1.28 & 2.29 & 5.60 & 1.30 & 0.60 & 0.14 & 291 & {$[48]$} \\
\hline & 4 & 1.04 & 2.09 & 5.92 & 0.57 & 1.51 & 0.71 & $293-523$ & [78] \\
\hline Average & $380^{*}$ & 2.25 & 2.07 & 7.24 & 2.87 & 4.83 & 12.0 & & \\
\hline \multirow{18}{*}{ Argon } & 22 & 2.37 & 2.77 & 7.56 & 2.17 & 2.23 & 2.14 & 50-973 & [26] \\
\hline & 63 & 2.78 & 2.25 & 5.85 & 2.38 & 2.98 & 1.94 & $90-500$ & [79] \\
\hline & 28 & 1.63 & 1.99 & 6.96 & 1.73 & 1.48 & 1.06 & $87-1000$ & [29] \\
\hline & 19 & 2.45 & 2.04 & 6.46 & 2.02 & 1.55 & 8.98 & $173-1597$ & {$[80]$} \\
\hline & 1 & 1.71 & 0.48 & 6.00 & 0.18 & 0.17 & 0.02 & 298 & {$[32]$} \\
\hline & 8 & 1.14 & 2.59 & 8.39 & 2.39 & 0.58 & 1.47 & $298-973$ & {$[64]$} \\
\hline & 11 & 0.88 & 2.64 & 8.05 & 1.96 & 0.87 & 0.90 & $298-473$ & {$[33]$} \\
\hline & 6 & 1.20 & 2.92 & 8.25 & 2.23 & 0.97 & 0.61 & $298-767$ & {$[81]$} \\
\hline & 9 & 1.48 & 3.43 & 8.85 & 2.86 & 1.20 & 3.30 & $298-1124$ & {$[31]$} \\
\hline & 8 & 1.17 & 3.03 & 8.45 & 2.43 & 0.63 & 1.54 & $298-973$ & {$[35]$} \\
\hline & 7 & 1.21 & 2.97 & 8.24 & 2.36 & 0.73 & 1.41 & 298-973 & {$[65]$} \\
\hline & 5 & 0.92 & 1.99 & 7.36 & 1.33 & 0.95 & 0.62 & $298-473$ & {$[82]$} \\
\hline & 5 & 0.85 & 1.95 & 7.36 & 1.29 & 0.92 & 0.60 & $300-473$ & {$[83]$} \\
\hline & 2 & 8.16 & 6.93 & 11.4 & 5.32 & 6.43 & 7.39 & $55-90$ & {$[84]$} \\
\hline & 1 & 2.01 & 0.12 & 5.79 & 0.34 & 0.31 & 0.07 & 273 & [85] \\
\hline & 1 & 3.90 & 1.56 & 4.81 & 1.25 & 6.76 & 26.7 & 1373 & {$[86]$} \\
\hline & 1 & 1.98 & 0.21 & 5.77 & 0.41 & 0.40 & 0.19 & 293 & [87] \\
\hline & 1 & 2.99 & 0.62 & 5.40 & 0.89 & 5.27 & 12.3 & 1100 & {$[40-42]$} \\
\hline
\end{tabular}


TABle 3: Continued.

\begin{tabular}{|c|c|c|c|c|c|c|c|c|c|}
\hline \multirow{2}{*}{ Compound } & \multirow{2}{*}{ Number of data points } & \multicolumn{6}{|c|}{ Average $\mathrm{RMSD}_{\mathrm{r}}$ in $\eta(\%)$} & \multirow[b]{2}{*}{$\Delta T(\mathrm{~K})$} & \multirow[b]{2}{*}{ Reference } \\
\hline & & Present study & L-J & Kihara & Lucas & Simsci & Refprop & & \\
\hline & 1 & 5.90 & 3.55 & 3.33 & 2.25 & 9.28 & 80.4 & 1873 & {$[88]$} \\
\hline & 9 & 0.94 & 2.79 & 8.22 & 2.08 & 0.80 & 0.49 & $298-773$ & {$[38]$} \\
\hline & 6 & 1.18 & 2.84 & 8.17 & 2.16 & 0.85 & 0.45 & $298-770$ & [89] \\
\hline & 8 & 0.93 & 2.68 & 8.07 & 1.95 & 0.93 & 0.46 & $298-673$ & {$[37]$} \\
\hline & 21 & 2.21 & 0.83 & 5.73 & 0.83 & 0.89 & 0.39 & $202-394$ & {$[90]$} \\
\hline & 5 & 12.8 & 13.9 & 17.4 & 13.6 & 12.2 & 11.4 & $775-1053$ & {$[40-42]$} \\
\hline & 7 & 12.0 & 12.7 & 15.9 & 12.5 & 11.7 & 29.9 & $775-1838$ & {$[76]$} \\
\hline & 5 & 3.75 & 1.70 & 5.30 & 1.20 & 5.79 & 36.5 & $569-1838$ & [91] \\
\hline & 6 & 5.64 & 4.66 & 8.52 & 3.63 & 3.25 & 3.96 & $72-291$ & {$[50]$} \\
\hline & 2 & 1.79 & 0.44 & 11.2 & 0.27 & 0.26 & 0.11 & $293-303$ & {$[48]$} \\
\hline & 1 & 2.19 & 0.01 & 5.59 & 0.60 & 0.58 & 0.37 & 291 & {$[45,46]$} \\
\hline & 5 & 5.22 & 4.39 & 8.44 & 3.36 & 3.00 & 3.72 & $72-291$ & [77] \\
\hline & 4 & 2.00 & 0.48 & 5.92 & 0.55 & 1.08 & 1.46 & $293-523$ & {$[52]$} \\
\hline & 2 & 1.72 & 0.50 & 6.00 & 0.23 & 0.22 & 0.09 & $293-303$ & [53] \\
\hline & 2 & 1.90 & 0.31 & 5.83 & 0.37 & 0.36 & 0.20 & $293-303$ & {$[47]$} \\
\hline & 8 & 2.09 & 0.49 & 5.83 & 0.64 & 1.10 & 1.47 & $293-523$ & {$[55]$} \\
\hline & 46 & 13.3 & 13.4 & 15.0 & 13.3 & 13.3 & 13.3 & $90-1000$ & {$[56]$} \\
\hline & 124 & 1.55 & 2.99 & 8.44 & 2.59 & 0.90 & 6.64 & $100-1600$ & [57] \\
\hline & 68 & 2.21 & 1.35 & 5.62 & 1.55 & 2.17 & 1.04 & $87-700$ & [58] \\
\hline & 135 & 1.53 & 2.67 & 7.81 & 2.21 & 1.46 & 1.34 & $80-1000$ & {$[92]$} \\
\hline & 63 & 1.60 & 1.43 & 6.50 & 1.13 & 1.50 & 0.57 & $90-500$ & [93] \\
\hline & 1 & 1.52 & 0.66 & 6.17 & 0.01 & 0.00 & 0.14 & 300 & {$[94]$} \\
\hline & 8 & 1.17 & 3.04 & 8.81 & 2.44 & 0.64 & 1.53 & $298-973$ & [59] \\
\hline & 11 & 1.65 & 1.53 & 6.50 & 1.24 & 1.43 & 0.56 & $100-600$ & {$[60]$} \\
\hline & 20 & 4.41 & 3.96 & 8.23 & 3.19 & 3.18 & 3.52 & $60-1000$ & {$[61]$} \\
\hline & 7 & 0.85 & 1.43 & 7.06 & 0.82 & 1.29 & 2.98 & $250-1000$ & {$[62]$} \\
\hline & 5 & 2.19 & 2.35 & 7.11 & 1.97 & 1.7 & 1.72 & $323-523$ & [95] \\
\hline & 5 & 0.81 & 1.48 & 6.98 & 0.76 & 0.58 & 0.47 & $298-423$ & [96] \\
\hline & 4 & 1.59 & 1.10 & 6.34 & 0.59 & 0.50 & 0.38 & 298-373 & [97] \\
\hline Average & $787^{*}$ & 2.75 & 3.14 & 7.85 & 2.84 & 2.44 & 3.76 & & \\
\hline \multirow{19}{*}{ Krypton } & 29 & 3.41 & 6.47 & 8.26 & 3.40 & 4.00 & 59.7 & $50-3273$ & {$[26]$} \\
\hline & 6 & 2.96 & 4.24 & 6.53 & 1.85 & 1.84 & 3.40 & $100-600$ & [29] \\
\hline & 1 & 3.90 & 1.95 & 4.82 & 0.83 & 0.50 & 0.91 & 293 & [73] \\
\hline & 2 & 3.40 & 2.59 & 5.36 & 0.58 & 0.51 & 1.53 & $293-300$ & {$[30]$} \\
\hline & 8 & 1.70 & 6.11 & 7.76 & 2.28 & 1.88 & 3.05 & 298-973 & {$[64]$} \\
\hline & 6 & 1.92 & 5.77 & 7.54 & 2.15 & 1.83 & 3.18 & $298-767$ & {$[81]$} \\
\hline & 8 & 1.89 & 6.25 & 7.86 & 2.51 & 2.07 & 3.20 & 298-973 & {$[65]$} \\
\hline & 8 & 1.88 & 6.07 & 7.67 & 2.25 & 1.83 & 2.97 & 298-973 & {$[35]$} \\
\hline & 9 & 1.60 & 6.61 & 8.20 & 2.80 & 2.40 & 3.27 & $298-1151$ & {$[31]$} \\
\hline & 9 & 1.87 & 5.82 & 7.53 & 2.05 & 1.71 & 3.10 & $298-778$ & {$[38]$} \\
\hline & 6 & 1.98 & 5.68 & 7.45 & 2.06 & 1.75 & 3.09 & $298-770$ & [89] \\
\hline & 1 & 5.59 & 0.36 & 3.27 & 2.47 & 2.13 & 0.70 & 293 & {$[67,68]$} \\
\hline & 1 & 5.08 & 0.84 & 3.74 & 1.97 & 1.64 & 0.21 & 293 & {$[66]$} \\
\hline & 1 & 5.42 & 0.52 & 3.42 & 2.30 & 1.95 & 0.54 & 293 & {$[76]$} \\
\hline & 12 & 2.01 & 5.43 & 7.27 & 1.83 & 1.57 & 2.93 & $298-773$ & [98] \\
\hline & 4 & 2.59 & 3.19 & 6.23 & 1.14 & 1.28 & 2.56 & $237-308$ & [49] \\
\hline & 2 & 4.93 & 0.82 & 3.82 & 1.96 & 1.63 & 0.43 & $273-293$ & {$[52]$} \\
\hline & 2 & 3.80 & 2.13 & 4.93 & 0.70 & 0.37 & 1.02 & $273-293$ & [54] \\
\hline & 2 & 3.72 & 2.10 & 4.40 & 0.69 & 0.68 & 1.10 & $273-293$ & {$[45,46]$} \\
\hline
\end{tabular}


TABLE 3: Continued.

\begin{tabular}{|c|c|c|c|c|c|c|c|c|c|}
\hline \multirow{2}{*}{ Compound } & \multirow{2}{*}{ Number of data points } & \multicolumn{6}{|c|}{ Average $\mathrm{RMSD}_{\mathrm{r}}$ in $\eta(\%)$} & \multirow[b]{2}{*}{$\Delta T(\mathrm{~K})$} & \multirow[b]{2}{*}{ Reference } \\
\hline & & Present study & L-J & Kihara & Lucas & Simsci & Refprop & & \\
\hline & 1 & 4.34 & 1.49 & 3.82 & 1.27 & 0.90 & 0.48 & 293 & {$[47]$} \\
\hline & 15 & 2.54 & 5.66 & 7.18 & 2.0 & 1.87 & 5.45 & $293-1600$ & {$[56]$} \\
\hline & 78 & 2.12 & 5.77 & 7.43 & 2.06 & 1.69 & 2.60 & $300-1300$ & {$[57]$} \\
\hline & 71 & 4.64 & 4.98 & 7.24 & 3.83 & 3.77 & 4.28 & $119-950$ & [58] \\
\hline & 235 & 2.02 & 6.98 & 8.64 & 3.61 & 3.57 & 12.5 & $80-2000$ & {$[92]$} \\
\hline & 51 & 8.49 & 7.44 & 8.66 & 7.50 & 7.45 & 7.40 & $125-500$ & {$[93]$} \\
\hline & 8 & 1.86 & 6.08 & 7.69 & 2.26 & 1.84 & 2.99 & $298-973$ & [59] \\
\hline & 10 & 4.62 & 2.00 & 4.19 & 2.0 & 2.09 & 0.26 & $150-600$ & {$[60]$} \\
\hline & 26 & 1.89 & 6.29 & 7.95 & 3.19 & 3.20 & 13.4 & $140-2000$ & {$[61]$} \\
\hline Average & $612^{*}$ & 3.03 & 6.18 & 7.94 & 3.42 & 3.32 & 10.2 & & \\
\hline \multirow{17}{*}{ Xenon } & 22 & 5.51 & 7.31 & 5.84 & 2.10 & 9.51 & 12.3 & $50-973$ & {$[26]$} \\
\hline & 6 & 5.06 & 6.39 & 5.33 & 1.86 & 6.59 & 12.0 & $100-600$ & [29] \\
\hline & 17 & 2.09 & 4.22 & 4.46 & 2.18 & 1.34 & 6.71 & $173-797$ & {$[80]$} \\
\hline & 8 & 3.13 & 7.60 & 5.87 & 1.42 & 2.11 & 7.94 & $298-973$ & {$[35]$} \\
\hline & 9 & 3.04 & 7.43 & 5.86 & 1.34 & 2.24 & 4.06 & $298-778$ & {$[38]$} \\
\hline & 7 & 1.24 & 2.65 & 3.75 & 2.51 & 0.46 & 5.94 & $202-298$ & [90] \\
\hline & 4 & 3.75 & 5.22 & 6.18 & 2.20 & 3.08 & 2.30 & $237-308$ & [49] \\
\hline & 3 & 0.53 & 2.62 & 2.99 & 2.32 & 0.74 & 2.90 & $273-373$ & [99] \\
\hline & 2 & 0.26 & 2.42 & 3.24 & 2.83 & 0.66 & 3.45 & 291 & {$[45,46]$} \\
\hline & 9 & 2.45 & 6.88 & 5.27 & 1.07 & 1.42 & 8.06 & $293-1000$ & {$[56]$} \\
\hline & 41 & 2.88 & 7.43 & 5.61 & 1.22 & 1.72 & 16.1 & $300-1300$ & [57] \\
\hline & 67 & 2.32 & 5.41 & 4.68 & 1.97 & 1.32 & 12.0 & $165-1250$ & {$[58]$} \\
\hline & 130 & 3.77 & 6.66 & 9.69 & 1.43 & 3.44 & 8.68 & $105-1000$ & [92] \\
\hline & 47 & 3.54 & 5.07 & 5.27 & 3.74 & 3.34 & 6.56 & $170-500$ & [93] \\
\hline & 8 & 3.11 & 7.52 & 5.77 & 1.60 & 2.08 & 7.96 & $298-973$ & [59] \\
\hline & 9 & 1.99 & 3.64 & 3.42 & 3.04 & 1.80 & 3.59 & $200-600$ & {$[60]$} \\
\hline & 14 & 2.45 & 5.87 & 4.93 & 1.85 & 1.42 & 8.25 & $180-1000$ & {$[61]$} \\
\hline Average & $403^{*}$ & 3.21 & 6.10 & 6.57 & 1.93 & 2.87 & 9.44 & & \\
\hline Overall & $2597^{* *}$ & 3.09 & 5.06 & 6.92 & 3.15 & 3.31 & 9.19 & & \\
\hline
\end{tabular}

${ }^{*}$ Number of data points for each noble gas. ${ }^{* *}$ Total number of data points for all noble gases.

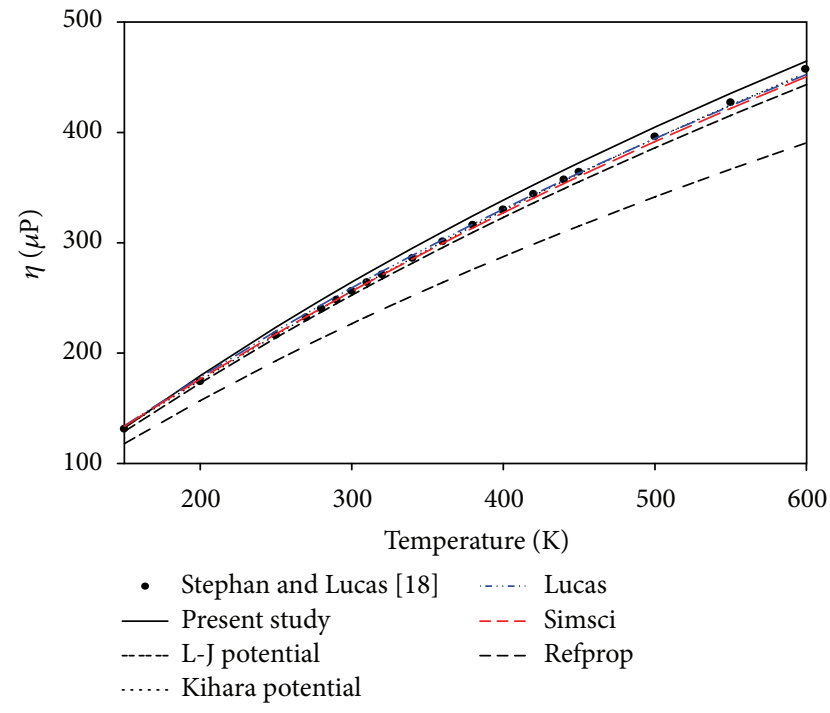

FIGURE 9: Comparison of measured and calculated viscosities for Kr.
Comparisons of the measured and calculated viscosities for pure noble gases ( $\mathrm{He}, \mathrm{Ne}, \mathrm{Ar}, \mathrm{Kr}$, and $\mathrm{Xe}$ ) are presented in Figures 6, 7, 8, 9, and 10, in order. Using the same set of potential parameters, other 2597 viscosity data available in the literature other than Stephan and Lucas [18] were reproduced with an overall average $\mathrm{RMSD}_{\mathrm{r}} 3.09 \%$ for all noble gases, noting that these results are more accurate than other existing investigations; original Lennard-Jones (12-6) potential $5.06 \%$, Kihara potential with group contribution method 6.92\%, Lucas method 3.15\%, Simsci correlations $3.31 \%$, and Refprop correlations $9.19 \%$.

It is mentioned here that for each dilute noble gas at $0.1 \mathrm{MPa}$ pressure, the Refprop Database provides selected viscosity data valid in specific temperature ranges, for example, 4-2219 K for helium, 27-1037 K for neon, 87-2992 K for argon, $119-1124 \mathrm{~K}$ for krypton, and $164-1100 \mathrm{~K}$ for xenon. In this work, their recommended viscosity data were fitted separately for each substance by least-squares analysis to obtain smoothing functions, usually reciprocal temperature expressions of third order, which were used to represent 
TABLE 4: Deviations between experimental and calculated thermal conductivities of pure noble gases.

\begin{tabular}{|c|c|c|c|c|c|c|c|}
\hline \multirow{2}{*}{ Compound } & \multirow{2}{*}{ Number of points } & \multicolumn{4}{|c|}{ Average $\mathrm{RMSD}_{\mathrm{r}}$ in $\lambda(\%)$} & \multirow[b]{2}{*}{$\Delta T(\mathrm{~K})$} & \multirow[b]{2}{*}{ Reference } \\
\hline & & Present study & L-J & Simsci & Refprop & & \\
\hline \multirow{25}{*}{ Helium } & 64 & 7.77 & 10.7 & 2.94 & 3.52 & $70-1000$ & {$[56]$} \\
\hline & 8 & 2.61 & 5.16 & 0.78 & 1.03 & $298-1010$ & {$[31]$} \\
\hline & 8 & 2.33 & 4.40 & 1.68 & 1.32 & $298-973$ & {$[34]$} \\
\hline & 8 & 2.41 & 4.42 & 1.75 & 1.27 & $298-973$ & {$[36]$} \\
\hline & 27 & 5.04 & 7.95 & 1.09 & 1.81 & $275-725$ & [57] \\
\hline & 93 & 6.10 & 9.10 & 7.20 & 20.1 & $20-1000$ & {$[58]$} \\
\hline & 8 & 2.47 & 4.67 & 1.30 & 0.95 & $298-973$ & {$[59]$} \\
\hline & 4 & 10.2 & 13.5 & 4.98 & 4.16 & $303-363$ & {$[100]$} \\
\hline & 2 & 7.72 & 11.2 & 2.35 & 1.25 & 303 & {$[101]$} \\
\hline & 25 & 3.66 & 6.21 & 1.41 & 4.52 & $50-973$ & {$[26]$} \\
\hline & 6 & 4.01 & 6.76 & 1.19 & 1.91 & $100-600$ & [29] \\
\hline & 11 & 4.95 & 7.65 & 1.44 & 1.95 & $100-600$ & {$[60]$} \\
\hline & 29 & 6.90 & 9.60 & 2.50 & 2.80 & $80-1000$ & {$[102]$} \\
\hline & 102 & 6.80 & 9.50 & 4.70 & 12.5 & $20-1000$ & [103] \\
\hline & 14 & 0.41 & 0.64 & 0.24 & 0.31 & $350-1000$ & [104] \\
\hline & 6 & 7.94 & 11.0 & 3.37 & 3.18 & $303-589$ & [105] \\
\hline & 52 & 5.90 & 8.60 & 5.50 & 17.4 & $20-1000$ & {$[106]$} \\
\hline & 5 & 11.0 & 14.0 & 6.88 & 7.20 & $250-600$ & {$[62]$} \\
\hline & 1 & 5.40 & 8.76 & 0.09 & 1.04 & 596 & [107] \\
\hline & 1 & 7.24 & 10.3 & 1.72 & 0.53 & 291 & {$[108]$} \\
\hline & 4 & 10.4 & 13.6 & 5.11 & 4.19 & $308-363$ & [109] \\
\hline & 2 & 4.68 & 7.32 & 1.32 & 0.07 & $302-793$ & [110] \\
\hline & 1 & 6.33 & 9.56 & 0.92 & 0.09 & 311 & [111] \\
\hline & 1 & 6.57 & 10.0 & 1.20 & 0.08 & 302 & [112] \\
\hline & 13 & 2.16 & 4.39 & 1.06 & 0.29 & $373-973$ & [63] \\
\hline Average & $495^{*}$ & 5.90 & 8.59 & 3.94 & 9.47 & & \\
\hline \multirow{21}{*}{ Neon } & 8 & 1.52 & 0.62 & 1.07 & 2.91 & $298-973$ & [64] \\
\hline & 8 & 2.50 & 1.16 & 0.66 & 3.87 & 298-973 & {$[65]$} \\
\hline & 1 & 1.04 & 0.54 & 1.10 & 2.53 & 291 & [47] \\
\hline & 8 & 2.74 & 1.41 & 0.66 & 4.11 & $298-973$ & [36] \\
\hline & 29 & 3.50 & 2.53 & 0.94 & 4.11 & $90-1300$ & {$[56]$} \\
\hline & 44 & 1.36 & 0.86 & 1.65 & 2.40 & $275-1275$ & [57] \\
\hline & 111 & 2.83 & 2.65 & 5.95 & 12.7 & $27-1000$ & {$[58]$} \\
\hline & 8 & 2.55 & 1.17 & 0.76 & 3.92 & $298-973$ & [59] \\
\hline & 4 & 1.67 & 3.25 & 2.10 & 0.84 & $303-363$ & {$[100]$} \\
\hline & 3 & 1.25 & 0.76 & 1.02 & 2.14 & $313-363$ & {$[113]$} \\
\hline & 22 & 2.40 & 1.70 & 2.40 & 4.36 & $50-973$ & [26] \\
\hline & 6 & 1.81 & 1.34 & 1.09 & 3.25 & $100-600$ & [29] \\
\hline & 11 & 1.48 & 3.03 & 2.50 & 2.04 & $100-600$ & {$[60]$} \\
\hline & 15 & 2.92 & 1.63 & 0.53 & 4.01 & $273-1100$ & [102] \\
\hline & 111 & 2.17 & 2.35 & 1.59 & 4.48 & $27-1500$ & [114] \\
\hline & 24 & 2.92 & 1.41 & 1.01 & 5.94 & $350-1500$ & [104] \\
\hline & 4 & 1.39 & 2.96 & 1.50 & 0.37 & $303-318$ & [105] \\
\hline & 1 & 1.36 & 2.90 & 1.53 & 0.14 & 311 & [115] \\
\hline & 1 & 0.20 & 1.39 & 0.32 & 1.73 & 296 & [107] \\
\hline & 1 & 1.04 & 0.53 & 1.11 & 2.54 & 291 & [108] \\
\hline & 2 & 5.86 & 4.27 & 4.28 & 7.50 & $302-793$ & [110] \\
\hline Average & $422^{*}$ & 2.41 & 2.08 & 2.63 & 6.20 & & \\
\hline
\end{tabular}


TABle 4: Continued.

\begin{tabular}{|c|c|c|c|c|c|c|c|}
\hline \multirow{2}{*}{ Compound } & \multirow{2}{*}{ Number of points } & \multicolumn{4}{|c|}{ Average $\mathrm{RMSD}_{\mathrm{r}}$ in $\lambda(\%)$} & \multirow[b]{2}{*}{$\Delta T(\mathrm{~K})$} & \multirow[b]{2}{*}{ Reference } \\
\hline & & Present study & L-J & Simsci & Refprop & & \\
\hline \multirow{35}{*}{ Argon } & 21 & 2.09 & 2.72 & 1.07 & 1.00 & $202-394$ & {$[90]$} \\
\hline & 3 & 2.15 & 3.97 & 0.48 & 5.08 & $90-273$ & {$[108]$} \\
\hline & 1 & 1.75 & 2.95 & 4.90 & 4.23 & 1373 & {$[116]$} \\
\hline & 1 & 0.14 & 4.35 & 1.09 & 2.98 & 291 & {$[47]$} \\
\hline & 9 & 1.06 & 5.27 & 0.53 & 1.24 & $298-1124$ & {$[31]$} \\
\hline & 8 & 1.21 & 5.11 & 0.49 & 1.17 & $298-973$ & {$[64]$} \\
\hline & 8 & 1.53 & 5.71 & 0.66 & 0.99 & $298-973$ & {$[65]$} \\
\hline & 8 & 1.48 & 5.66 & 0.77 & 1.09 & $298-973$ & {$[35]$} \\
\hline & 66 & 1.76 & 4.50 & 1.25 & 1.60 & $90-2000$ & {$[56]$} \\
\hline & 14 & 2.25 & 6.41 & 2.36 & 2.57 & $331-645$ & [117] \\
\hline & 97 & 1.74 & 4.98 & 0.80 & 0.99 & $280-1390$ & [57] \\
\hline & 122 & 2.55 & 6.17 & 1.26 & 5.23 & $87-3300$ & {$[58]$} \\
\hline & 235 & 2.10 & 5.97 & 1.02 & 2.20 & $80-2000$ & {$[92]$} \\
\hline & 63 & 2.74 & 3.09 & 3.08 & 5.05 & $90-500$ & [79] \\
\hline & 63 & 3.82 & 4.80 & 3.40 & 3.53 & $90-500$ & {$[93]$} \\
\hline & 8 & 1.36 & 5.50 & 0.54 & 1.10 & $298-973$ & [59] \\
\hline & 3 & 0.77 & 4.70 & 1.37 & 2.70 & $313-363$ & [113] \\
\hline & 29 & 2.63 & 5.64 & 2.48 & 7.10 & $50-3273$ & {$[26]$} \\
\hline & 6 & 1.92 & 4.11 & 2.46 & 5.20 & $100-600$ & [29] \\
\hline & 11 & 1.65 & 3.37 & 1.39 & 3.07 & $100-600$ & {$[60]$} \\
\hline & 54 & 2.02 & 5.78 & 1.07 & 2.55 & $80-2000$ & [102] \\
\hline & 118 & 1.67 & 4.82 & 1.21 & 2.14 & $88-2500$ & {$[103]$} \\
\hline & 24 & 1.70 & 6.20 & 0.50 & 0.90 & $350-1500$ & [104] \\
\hline & 4 & 2.73 & 3.57 & 1.65 & 0.77 & $273-593$ & [105] \\
\hline & 4 & 0.49 & 4.15 & 0.68 & 2.20 & $308-363$ & {$[118]$} \\
\hline & 3 & 1.08 & 3.52 & 0.21 & 1.81 & $300-340$ & [119] \\
\hline & 8 & 0.79 & 4.42 & 1.20 & 2.51 & $295-420$ & {$[120]$} \\
\hline & 60 & 2.03 & 4.95 & 1.16 & 2.04 & $90-2000$ & [106] \\
\hline & 7 & 1.41 & 3.86 & 1.09 & 1.43 & $250-1000$ & {$[62]$} \\
\hline & 1 & 1.40 & 3.19 & 0.25 & 1.52 & 311 & [115] \\
\hline & 1 & 1.14 & 3.41 & 0.06 & 1.93 & 296 & [107] \\
\hline & 4 & 0.50 & 4.15 & 0.67 & 2.20 & $308-363$ & [109] \\
\hline & 2 & 3.10 & 7.10 & 2.40 & 2.70 & $302-793$ & {$[110]$} \\
\hline & 1 & 1.40 & 3.20 & 0.25 & 1.52 & 311 & [111] \\
\hline & 22 & 1.46 & 4.21 & 0.84 & 3.31 & $87-1000$ & [29] \\
\hline Average & $1089^{*}$ & 2.11 & 5.22 & 1.31 & 2.82 & & \\
\hline \multirow{14}{*}{ Krypton } & 9 & 1.90 & 14.8 & 0.82 & 2.93 & 298-1149 & [31] \\
\hline & 8 & 1.80 & 14.8 & 0.88 & 3.10 & 298-973 & {$[64]$} \\
\hline & 8 & 1.65 & 15.1 & 1.24 & 3.44 & 298-973 & {$[65]$} \\
\hline & 18 & 3.20 & 19.5 & 4.18 & 6.57 & $125-1300$ & [56] \\
\hline & 5 & 4.88 & 10.6 & 1.94 & 0.94 & 291-318 & [57] \\
\hline & 101 & 2.33 & 14.0 & 1.71 & 3.19 & 119-1900 & {$[58]$} \\
\hline & 6 & 3.12 & 13.4 & 2.25 & 3.45 & $100-600$ & {$[26]$} \\
\hline & 235 & 2.60 & 15.5 & 2.50 & 4.50 & $80-2000$ & {$[92]$} \\
\hline & 51 & 2.50 & 12.5 & 0.96 & 2.50 & $125-500$ & {$[93]$} \\
\hline & 8 & 1.75 & 14.9 & 1.10 & 3.28 & 298-973 & [59] \\
\hline & 3 & 0.81 & 15.5 & 3.68 & 5.50 & $313-363$ & [113] \\
\hline & 27 & 3.50 & 14.6 & 2.70 & 7.74 & $50-2273$ & {$[26]$} \\
\hline & 10 & 4.62 & 10.8 & 2.46 & 0.64 & $150-600$ & {$[60]$} \\
\hline & 109 & 5.80 & 14.4 & 5.33 & 6.25 & $120-2000$ & {$[114]$} \\
\hline
\end{tabular}


TABLE 4: Continued.

\begin{tabular}{|c|c|c|c|c|c|c|c|}
\hline \multirow{2}{*}{ Compound } & \multirow{2}{*}{ Number of points } & \multicolumn{4}{|c|}{ Average $\mathrm{RMSD}_{\mathrm{r}}$ in $\lambda(\%)$} & \multirow[b]{2}{*}{$\Delta T(\mathrm{~K})$} & \multirow[b]{2}{*}{ Reference } \\
\hline & & Present study & L-J & Simsci & Refprop & & \\
\hline & 24 & 3.00 & 15.9 & 2.50 & 3.50 & $350-1500$ & {$[104]$} \\
\hline & 5 & 5.76 & 9.80 & 2.67 & 0.78 & $303-318$ & {$[105]$} \\
\hline & 4 & 4.24 & 11.3 & 1.35 & 1.06 & $308-363$ & [118] \\
\hline & 1 & 4.38 & 11.0 & 1.30 & 0.65 & 311 & {$[115]$} \\
\hline & 1 & 4.16 & 10.9 & 1.17 & 0.81 & 291 & [108] \\
\hline & 4 & 4.21 & 11.3 & 1.34 & 1.11 & $308-363$ & [109] \\
\hline & 2 & 4.06 & 17.4 & 5.03 & 6.98 & $302-793$ & {$[110]$} \\
\hline Average & $639^{*}$ & 3.22 & 14.7 & 2.70 & 4.35 & & \\
\hline \multirow{21}{*}{ Xenon } & 7 & 1.29 & 14.2 & 9.68 & 2.32 & $202-298$ & {$[90]$} \\
\hline & 8 & 3.28 & 14.2 & 2.65 & 1.43 & $298-973$ & {$[35]$} \\
\hline & 24 & 7.30 & 17.7 & 1.02 & 4.55 & $175-1300$ & {$[56]$} \\
\hline & 1 & 3.53 & 12.7 & 0.60 & 1.95 & 291 & [57] \\
\hline & 1 & 3.36 & 12.6 & 0.77 & 1.78 & 291 & [108] \\
\hline & 134 & 5.36 & 15.7 & 5.80 & 10.1 & $165-5000$ & {$[58]$} \\
\hline & 230 & 4.87 & 15.3 & 4.83 & 1.96 & $105-2000$ & [92] \\
\hline & 47 & 3.33 & 12.0 & 2.10 & 0.71 & $170-500$ & [93] \\
\hline & 8 & 3.29 & 14.2 & 2.64 & 1.43 & $298-973$ & [59] \\
\hline & 4 & 3.39 & 13.1 & 0.57 & 2.48 & $303-363$ & [100] \\
\hline & 3 & 8.35 & 17.6 & 4.75 & 7.55 & $313-363$ & {$[113]$} \\
\hline & 29 & 5.84 & 14.9 & 8.18 & 2.90 & $50-3273$ & {$[26]$} \\
\hline & 6 & 5.50 & 12.8 & 5.96 & 2.82 & $100-600$ & [29] \\
\hline & 9 & 1.46 & 11.2 & 3.60 & 0.92 & $200-600$ & {$[60]$} \\
\hline & 95 & 6.40 & 16.7 & 1.74 & 3.93 & $165-1500$ & {$[114]$} \\
\hline & 24 & 8.00 & 19.0 & 1.20 & 5.10 & $350-1500$ & {$[104]$} \\
\hline & 6 & 4.20 & 7.60 & 7.87 & 5.20 & $303-318$ & [105] \\
\hline & 67 & 5.64 & 16.1 & 5.97 & 10.2 & $165-5000$ & [106] \\
\hline & 2 & 9.00 & 19.0 & 3.90 & 7.01 & $302-793$ & {$[110]$} \\
\hline & 1 & 0.80 & 9.10 & 4.95 & 2.06 & 311 & [111] \\
\hline & 1 & 7.30 & 16.3 & 3.43 & 6.00 & 302 & [112] \\
\hline Average & $707^{*}$ & 5.26 & 15.4 & 4.39 & 4.76 & & \\
\hline Overall & $3352^{* *}$ & 3.59 & 9.28 & 2.78 & 4.93 & & \\
\hline
\end{tabular}

${ }^{*}$ Number of data points for each noble gas. ${ }^{* *}$ Total number of data points for all noble gases.

viscosity data at the same temperature as those of experimental data for the reasonable comparisons. Prediction results from the Refprop correlations are observed to be not in reliable agreement with measured viscosity data, especially near upper and lower limits of temperature ranges specified previously, as shown in Table 3. The Simsci Database [24] provides the smoothing viscosity function with four coefficients $c_{i}(i=1$ to 4$)$

$$
\eta=\frac{c_{1} T^{\mathcal{c}_{2}}}{\left(1+c_{3} / T+c_{4} / T\right)}
$$

valid in specific temperature ranges: $20-2000 \mathrm{~K}$ for helium, $30-3272 \mathrm{~K}$ for neon, $83-3273 \mathrm{~K}$ for argon, $100-1500 \mathrm{~K}$ for krypton, and $100-1600 \mathrm{~K}$ for xenon.

The next stage of this work is to calculate other properties such as thermal conductivity and self-diffusion coefficient, not used for parameter determinations, using the same set of potential parameters determined earlier. As shown in Table 4, the overall average $\mathrm{RMSD}_{\mathrm{r}}$ value of $3.59 \%$ between a total of 3352 experimental and calculated thermal conductivities obtained by the proposed model is somewhat less reliable to the Simsci correlations $2.78 \%$, but compares very well with the original Lennard-Jones (12-6) potential 9.28\% and the Refprop correlations $4.93 \%$.

Like the case of viscosities, the Refprop Database [22] provides dilute gas thermal conductivity data at $0.1 \mathrm{MPa}$ suitable in specific temperature ranges: $4-1100 \mathrm{~K}$ for helium, $27-1039 \mathrm{~K}$ for neon, $87-2968 \mathrm{~K}$ for argon, $119-1100 \mathrm{~K}$ for krypton, and $164-1101 \mathrm{~K}$ for xenon. The procedure to produce thermal conductivity data is the same as that of viscosity. The Simsci Database also provides the same type of soothing thermal conductivity function, (24), in specific temperature ranges: $30-2000 \mathrm{~K}$ for helium, $30-3272 \mathrm{~K}$ for neon, $90-$ $3273 \mathrm{~K}$ for argon, $120-2000 \mathrm{~K}$ for krypton, and $165-1500 \mathrm{~K}$ for xenon. Comparisons of the measured and calculated thermal conductivities for pure noble gases $(\mathrm{He}, \mathrm{Ne}, \mathrm{Ar}$, 
TABLE 5: Deviations between experimental and calculated self-diffusion coefficients of pure noble gases.

\begin{tabular}{|c|c|c|c|c|c|c|}
\hline \multirow{2}{*}{ Compound } & \multirow{2}{*}{ Number of points } & \multicolumn{3}{|c|}{ Average $\mathrm{RMSD}_{\mathrm{r}}$ in $D(\%)$} & \multirow{2}{*}{$\Delta T(\mathrm{~K})$} & \multirow{2}{*}{ Reference } \\
\hline & & Present study & L-J & Fuller & & \\
\hline \multicolumn{7}{|c|}{ Prediction results } \\
\hline \multirow{2}{*}{ Helium } & 17 & 4.72 & 2.04 & 10.6 & $50-623$ & {$[26]$} \\
\hline & 7 & 11.0 & 8.44 & 13.1 & $14-296$ & {$[56]$} \\
\hline Average & $24^{*}$ & 6.55 & 3.90 & 11.3 & & \\
\hline \multirow{4}{*}{ Neon } & 29 & 3.36 & 2.25 & 8.07 & $50-3273$ & {$[26]$} \\
\hline & 17 & 6.85 & 4.93 & 13.2 & $77-6000$ & {$[56]$} \\
\hline & 20 & 4.72 & 2.78 & 6.50 & $295-1219$ & {$[121]$} \\
\hline & 1 & 4.55 & 7.77 & 5.96 & 293 & {$[122]$} \\
\hline Average & $67^{*}$ & 4.67 & 3.17 & 8.88 & & \\
\hline \multirow{2}{*}{ Argon } & 29 & 2.87 & 4.70 & 10.9 & $50-323$ & {$[26]$} \\
\hline & 12 & 4.10 & 3.51 & 14.2 & $90-326$ & {$[56]$} \\
\hline \multirow[t]{2}{*}{ Average } & $41^{*}$ & 3.23 & 4.35 & 11.9 & & \\
\hline & 29 & 4.00 & 13.92 & 9.70 & $50-3273$ & {$[26]$} \\
\hline \multirow[t]{2}{*}{ Krypton } & 18 & 6.83 & 16.93 & 3.69 & $199-6000$ & {$[56]$} \\
\hline & 1 & 4.90 & 8.94 & 3.30 & 293 & [123] \\
\hline \multirow[t]{2}{*}{ Average } & $48^{*}$ & 5.08 & 14.95 & 7.31 & & \\
\hline & 29 & 6.27 & 13.9 & 16.2 & $50-3273$ & {$[26]$} \\
\hline \multirow[t]{2}{*}{ Xenon } & 27 & 6.02 & 16.9 & 13.1 & $194-15000$ & {$[56]$} \\
\hline & 1 & 1.06 & 7.40 & 17.2 & 293 & [123] \\
\hline Average & $57^{*}$ & 6.06 & 15.2 & 14.8 & & \\
\hline Overall & $237^{* *}$ & 5.03 & 8.73 & 10.7 & & \\
\hline
\end{tabular}

${ }^{*}$ Number of data points for each noble gas. ${ }^{* *}$ Total number of data points for all noble gases.

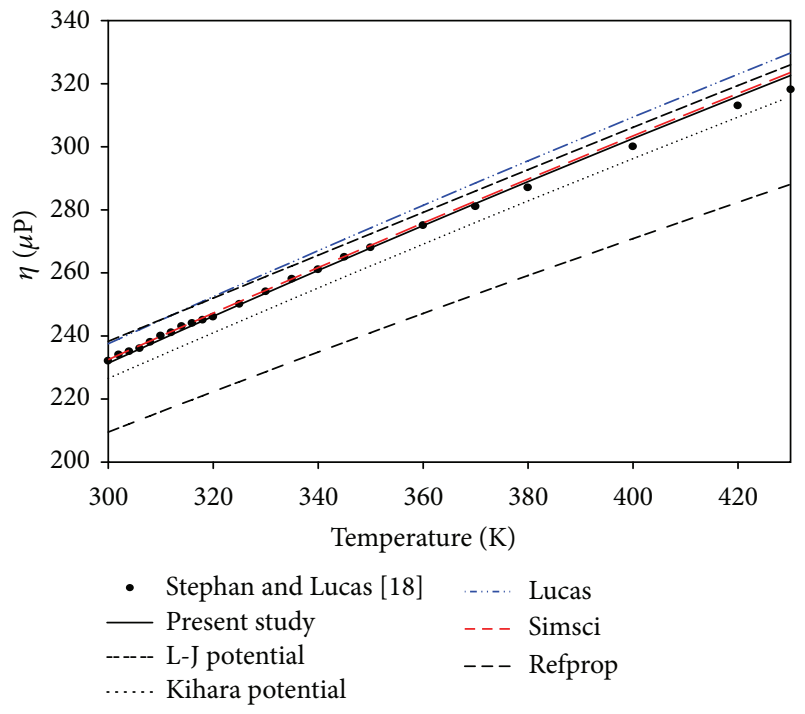

FIGURE 10: Comparison of measured and calculated viscosities for Xe.

$\mathrm{Kr}$, and $\mathrm{Xe}$ ) are depicted in Figures $11,12,13,14$, and 15, in order. As shown in Figures 14 and 15, Refprop Database shows considerably larger discrepancies between measured and calculated thermal conductivities for krypton and xenon than other methods particularly at high-temperature region, at which recommended data is not reliable, as specified by them.

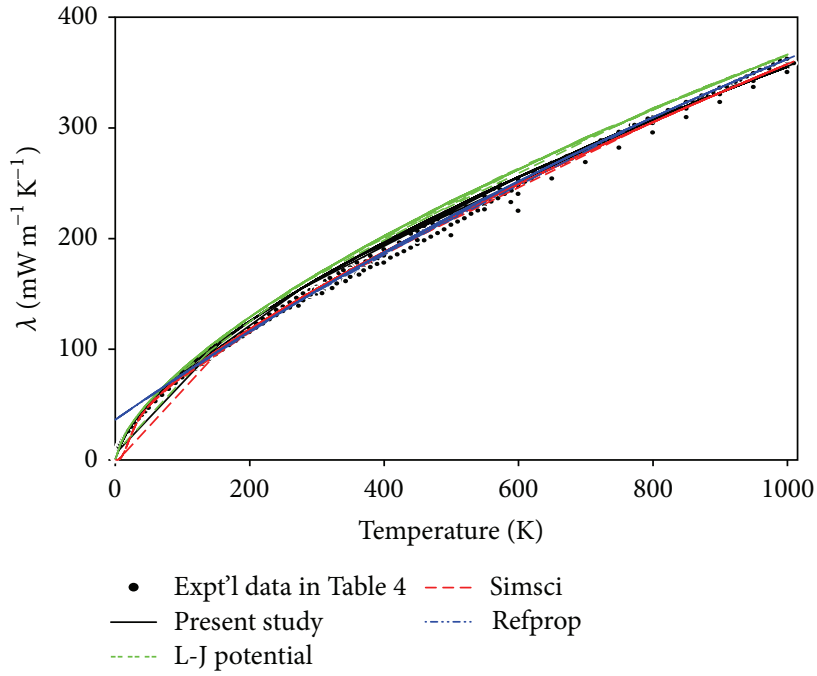

FIGURE 11: Comparison of measured and calculated thermal conductivities for He.

A total of 237 self-diffusion coefficient data were next tested. As shown in Table 5, the overall average RMSD ${ }_{\mathrm{r}}$ value of $5.03 \%$ from all noble gases was obtained by this work, in which the result is in better agreement with experimental data than those of the original Lennard-Jones (12-6) potential $8.73 \%$ and of the Fuller method [25] $10.7 \%$. It is indicated that for the helium and neon gas, the proposed method is less 
TABLE 6: Deviations between experimental and predicted second cross-virial coefficients of noble gas mixtures.

\begin{tabular}{|c|c|c|c|c|c|c|c|}
\hline \multirow{2}{*}{ Mixtures } & \multirow{2}{*}{ Number of points } & \multicolumn{4}{|c|}{ Average RMSD in $B_{12}\left(\mathrm{~cm}^{3} \mathrm{~mol}^{-1}\right)$} & \multirow{2}{*}{$\Delta T(\mathrm{~K})$} & \multirow{2}{*}{ Reference } \\
\hline & & Present study & L-J & Dymond et al. & Tsonopoulos & & \\
\hline \multicolumn{8}{|c|}{ Mixtures of noble gases } \\
\hline \multirow{2}{*}{ Helium-neon } & 18 & 2.05 & 5.82 & 0.94 & 9.40 & $15-323$ & {$[2]$} \\
\hline & 23 & 0.66 & 0.54 & 1.72 & 10.6 & $273-3273$ & [26] \\
\hline \multirow{2}{*}{ Helium-argon } & 21 & 2.03 & 0.92 & 0.44 & 6.85 & $90-773$ & {$[2]$} \\
\hline & 23 & 4.90 & 5.71 & 6.37 & 14.8 & $273-3273$ & {$[26]$} \\
\hline \multirow{2}{*}{ Helium-krypton } & 15 & 5.66 & 3.49 & 0.99 & 1.84 & $90-323$ & {$[2]$} \\
\hline & 23 & 8.54 & 12.0 & 12.0 & 15.9 & $273-3273$ & {$[26]$} \\
\hline \multirow{2}{*}{ Helium-xenon } & 9 & 10.9 & 6.07 & 0.71 & 7.14 & $120-323$ & {$[2]$} \\
\hline & 23 & 15.8 & 19.7 & 23.3 & 21.5 & $273-3273$ & {$[26]$} \\
\hline Average & $155^{*}$ & 6.15 & 7.12 & 6.75 & 11.9 & & \\
\hline \multirow{2}{*}{ Neon-argon } & 30 & 1.68 & 34.9 & 1.00 & 7.72 & $84-475$ & {$[2]$} \\
\hline & 23 & 3.15 & 1.15 & 6.69 & 4.71 & $273-3273$ & {$[26]$} \\
\hline \multirow{2}{*}{ Neon-krypton } & 48 & 5.15 & 48.6 & 2.76 & 14.3 & $100-475$ & {$[2]$} \\
\hline & 23 & 3.86 & 3.29 & 8.07 & 6.69 & $273-3273$ & {$[26]$} \\
\hline \multirow{2}{*}{ Neon-xenon } & 28 & 16.5 & 39.4 & 2.61 & 17.6 & $162-475$ & {$[2]$} \\
\hline & 23 & 10.8 & 7.36 & 18.9 & 12.5 & $273-3273$ & {$[26]$} \\
\hline Average & $175^{*}$ & 6.68 & 27.2 & 5.77 & 11.2 & & \\
\hline \multirow{2}{*}{ Argon-krypton } & 50 & 3.26 & 150.3 & 1.82 & 2.51 & $108-695$ & {$[2]$} \\
\hline & 23 & 1.58 & 16.7 & 2.93 & 4.53 & $273-3273$ & {$[26]$} \\
\hline \multirow{2}{*}{ Argon-xenon } & 5 & 12.3 & 125.5 & 0.29 & 12.1 & $173-323$ & {$[2]$} \\
\hline & 23 & 4.60 & 23.9 & 30.0 & 5.76 & $273-3273$ & {$[26]$} \\
\hline Average & $101^{*}$ & 3.63 & 89.9 & 8.43 & 4.18 & & \\
\hline \multirow{2}{*}{ Krypton-xenon } & 35 & 7.34 & 173.7 & 1.97 & 5.76 & $160-700$ & {$[2]$} \\
\hline & 23 & 2.34 & 42.6 & 3.40 & 4.44 & $273-3273$ & {$[26]$} \\
\hline Average & $58^{*}$ & 5.36 & 121.7 & 2.54 & 5.24 & & \\
\hline Overall & $489^{* *}$ & 5.73 & 45.0 & 6.25 & 9.28 & & \\
\hline
\end{tabular}

${ }^{*}$ Number of data points for each gas mixture. ${ }^{* *}$ Total number of data points for all gas mixtures.

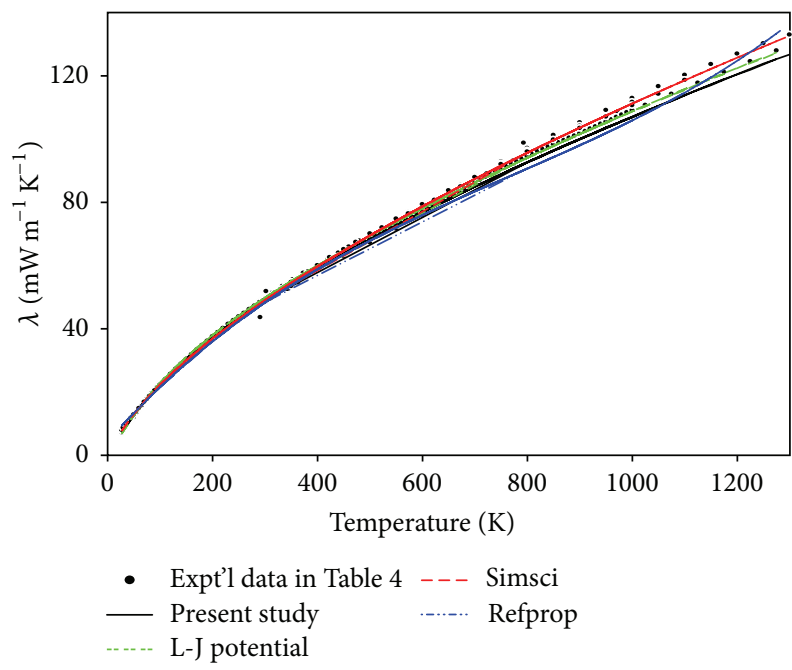

FIGURE 12: Comparison of measured and calculated thermal conductivities for Ne.

accurate than the original Lennard-Jones (12-6) potential. Comparisons of the measured and calculated self-diffusion

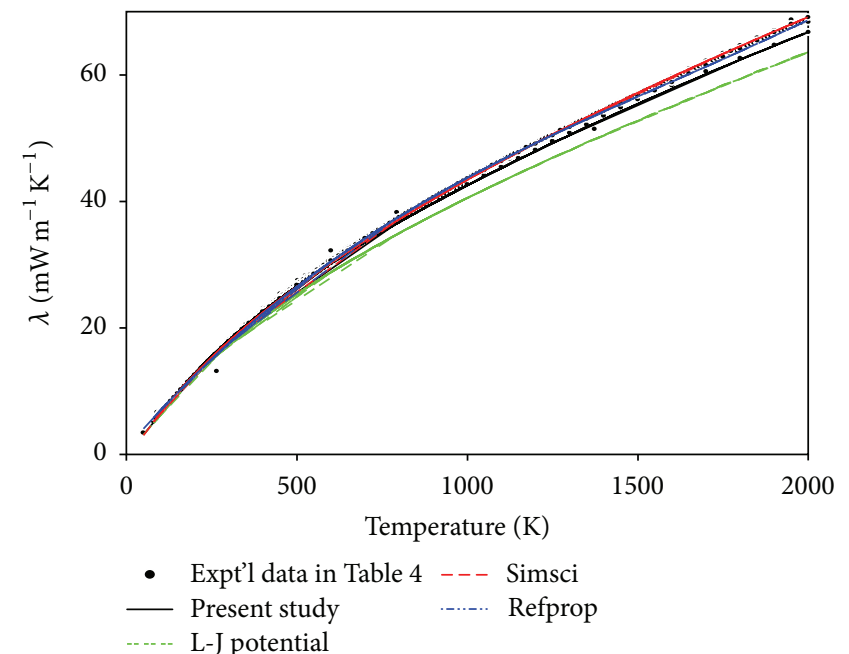

FIGURE 13: Comparison of measured and calculated thermal conductivities for Ar.

coefficients for noble gases ( $\mathrm{He}, \mathrm{Ne}, \mathrm{Ar}, \mathrm{Kr}$, and $\mathrm{Xe}$ ) are depicted in Figures 16, 17, 18, 19, and 20, in order. 
TABLE 7: Deviations between experimental and predicted mixture viscosities of noble gas mixtures.

\begin{tabular}{|c|c|c|c|c|c|c|}
\hline \multirow{2}{*}{ Mixtures } & \multirow{2}{*}{ Number of points } & \multicolumn{3}{|c|}{ Average $\mathrm{RMSD}_{\mathrm{r}}$ in $\eta_{\text {mix }}(\%)$} & \multirow{2}{*}{$\Delta T(\mathrm{~K})$} & \multirow{2}{*}{ Reference } \\
\hline & & Present study & L-J & Lucas method & & \\
\hline \multirow{9}{*}{ Helium-neon } & 87 & 6.24 & 13.6 & 8.85 & $50-3273$ & {$[26]$} \\
\hline & 7 & 2.40 & 4.87 & 3.56 & 291 & {$[47]$} \\
\hline & 10 & 1.93 & 4.93 & 4.39 & 293 & {$[53]$} \\
\hline & 32 & 1.75 & 4.77 & 8.18 & $298-973$ & {$[36]$} \\
\hline & 3 & 2.31 & 4.88 & 3.97 & 293 & {$[40-42]$} \\
\hline & 10 & 2.58 & 5.15 & 3.80 & $293-523$ & {$[124]$} \\
\hline & 11 & 3.36 & 4.82 & 6.98 & $293-523$ & {$[55]$} \\
\hline & 31 & 2.22 & 4.96 & 3.67 & $20-523$ & {$[56]$} \\
\hline & 162 & 2.10 & 8.40 & 7.49 & $100-950$ & {$[57]$} \\
\hline \multirow{13}{*}{ Helium-argon } & 87 & 4.49 & 7.31 & 4.95 & $50-3273$ & {$[26]$} \\
\hline & 12 & 3.38 & 4.25 & 3.51 & $293-303$ & {$[125]$} \\
\hline & 11 & 3.75 & 3.66 & 4.78 & 291 & {$[47]$} \\
\hline & 58 & 3.84 & 4.07 & 4.76 & $72-192$ & {$[50]$} \\
\hline & 40 & 2.98 & 6.06 & 3.63 & $298-993$ & {$[31]$} \\
\hline & 3 & 3.52 & 0.92 & 6.46 & 293 & {$[78]$} \\
\hline & 3 & 4.09 & 3.01 & 5.19 & 293 & {$[40-42]$} \\
\hline & 6 & 3.69 & 3.12 & 5.25 & $293-523$ & {$[55]$} \\
\hline & 16 & 6.72 & 7.04 & 5.47 & $293-523$ & {$[56]$} \\
\hline & 123 & 1.29 & 5.18 & 3.20 & $100-1500$ & {$[57]$} \\
\hline & 28 & 3.14 & 3.23 & 4.45 & 298 & {$[126]$} \\
\hline & 12 & 1.26 & 2.44 & 4.57 & $300-1100$ & {$[127]$} \\
\hline & 6 & 3.62 & 0.51 & 7.15 & 298 & {$[128]$} \\
\hline \multirow{5}{*}{ Helium-krypton } & 87 & 4.60 & 9.41 & 13.4 & $50-3273$ & {$[26]$} \\
\hline & 9 & 4.44 & 7.72 & 9.63 & 291 & {$[45,46]$} \\
\hline & 16 & 4.98 & 5.28 & 10.5 & 303 & {$[54]$} \\
\hline & 40 & 7.72 & 8.91 & 5.78 & $298-993$ & {$[31]$} \\
\hline & 135 & 4.07 & 11.8 & 4.94 & $300-1000$ & {$[57]$} \\
\hline \multirow{4}{*}{ Helium-xenon } & 87 & 3.85 & 9.63 & 13.9 & $50-3273$ & {$[26]$} \\
\hline & 10 & 3.41 & 6.11 & 19.2 & 291 & {$[45,46]$} \\
\hline & 18 & 2.50 & 8.85 & 13.6 & $298-778$ & {$[38]$} \\
\hline & 90 & 2.34 & 9.46 & 12.7 & $300-750$ & {$[57]$} \\
\hline Average & $1250^{*}$ & 3.52 & 8.34 & 8.33 & & \\
\hline \multirow{10}{*}{ Neon-argon } & 87 & 1.91 & 3.95 & 4.23 & $50-3273$ & {$[26]$} \\
\hline & 6 & 1.24 & 2.80 & 1.60 & 293 & {$[53]$} \\
\hline & 36 & 0.66 & 2.15 & 2.85 & $298-973$ & {$[64]$} \\
\hline & 26 & 3.87 & 4.34 & 4.24 & $72-291$ & {$[77]$} \\
\hline & 3 & 1.68 & 1.48 & 0.70 & 293 & {$[78]$} \\
\hline & 3 & 1.61 & 0.95 & 0.66 & 293 & {$[40-42]$} \\
\hline & 9 & 1.43 & 1.85 & 0.70 & 291 & {$[47]$} \\
\hline & 12 & 3.22 & 3.95 & 3.56 & $293-523$ & {$[55]$} \\
\hline & 16 & 2.56 & 1.59 & 1.14 & $293-523$ & {$[56]$} \\
\hline & 162 & 0.95 & 4.28 & 2.72 & $100-950$ & {$[57]$} \\
\hline \multirow{4}{*}{ Neon-krypton } & 87 & 2.63 & 10.3 & 3.25 & $50-3273$ & {$[26]$} \\
\hline & 9 & 1.28 & 8.04 & 2.44 & 291 & {$[45,46]$} \\
\hline & 40 & 0.85 & 9.63 & 1.60 & $298-973$ & {$[65]$} \\
\hline & 126 & 0.78 & 10.5 & 1.55 & $300-950$ & {$[57]$} \\
\hline
\end{tabular}


TABLe 7: Continued.

\begin{tabular}{|c|c|c|c|c|c|c|}
\hline \multirow{2}{*}{ Mixtures } & \multirow{2}{*}{ Number of points } & \multicolumn{3}{|c|}{ Average $\mathrm{RMSD}_{\mathrm{r}}$ in $\eta_{\text {mix }}(\%)$} & \multirow{2}{*}{$\Delta T(\mathrm{~K})$} & \multirow{2}{*}{ Reference } \\
\hline & & Present study & L-J & Lucas method & & \\
\hline \multirow{4}{*}{ Neon-xenon } & 87 & 4.70 & 11.1 & 3.94 & $50-3273$ & {$[26]$} \\
\hline & 8 & 0.85 & 7.30 & 6.42 & 291 & {$[45,46]$} \\
\hline & 12 & 2.46 & 8.69 & 3.74 & $298-773$ & {$[38]$} \\
\hline & 90 & 4.70 & 10.2 & 3.11 & $300-750$ & {$[57]$} \\
\hline Average & $819^{*}$ & 2.46 & 8.11 & 2.95 & & \\
\hline \multirow{4}{*}{ Argon-krypton } & 87 & 2.69 & 10.7 & 3.13 & $50-3273$ & {$[26]$} \\
\hline & 36 & 0.71 & 10.5 & 1.81 & $298-973$ & {$[64]$} \\
\hline & 8 & 2.20 & 8.40 & 1.76 & 291 & {$[45,46]$} \\
\hline & 189 & 0.97 & 10.8 & 2.30 & $300-1300$ & {$[57]$} \\
\hline \multirow{5}{*}{ Argon-xenon } & 87 & 4.07 & 11.3 & 2.84 & $50-3273$ & [26] \\
\hline & 9 & 0.64 & 8.04 & 4.01 & 291 & {$[45,46]$} \\
\hline & 12 & 1.87 & 9.15 & 1.61 & $298-773$ & {$[38]$} \\
\hline & 34 & 2.46 & 10.5 & 2.62 & $173-299$ & {$[80]$} \\
\hline & 189 & 2.71 & 13.2 & 1.42 & $173-1597$ & {$[57]$} \\
\hline Average & $651^{*}$ & 2.20 & 11.4 & 2.23 & & \\
\hline \multirow{4}{*}{ Krypton-xenon } & 87 & 3.97 & 14.5 & 2.71 & $50-3273$ & {$[26]$} \\
\hline & 9 & 1.22 & 11.2 & 2.19 & 291 & {$[45,46]$} \\
\hline & 12 & 2.18 & 14.1 & 1.18 & $298-773$ & {$[38]$} \\
\hline & 90 & 3.52 & 14.7 & 2.98 & $300-750$ & {$[57]$} \\
\hline Average & $198^{*}$ & 3.53 & 14.4 & 2.72 & & \\
\hline Overall & $2918^{* *}$ & 2.93 & 10.6 & 4.05 & & \\
\hline
\end{tabular}

${ }^{*}$ Number of data points for each gas mixture. ${ }^{* *}$ Total number of data points for all gas mixtures.

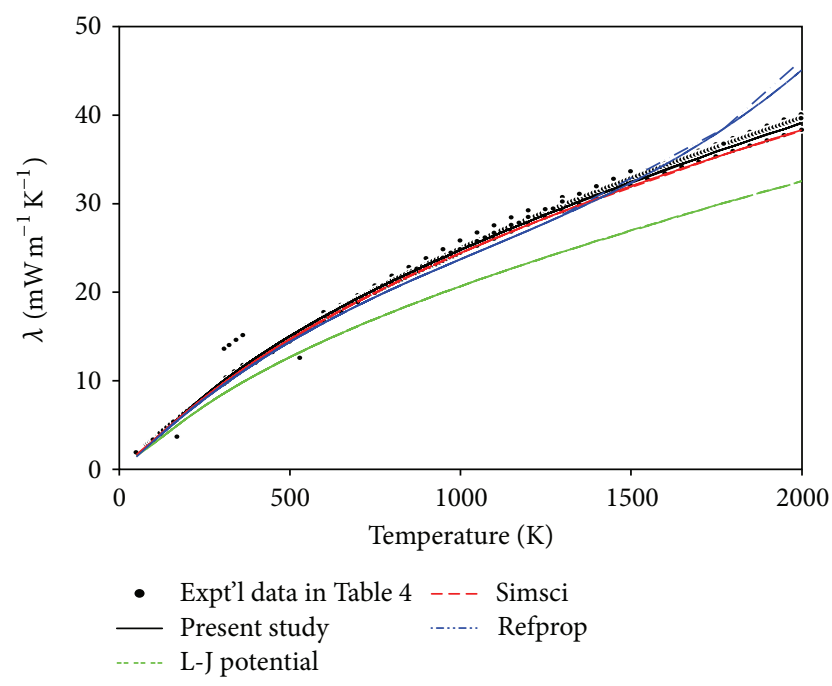

FIGURE 14: Comparison of measured and calculated thermal conductivities for Kr.

3.2. Noble Gas Mixtures. The same set of potential parameters estimated from pure gas information was applied to predict mixture properties such as second cross-virial coefficient, mixture viscosity, mixture thermal conductivity, and binary diffusion coefficient with no additional parameters.

Table 6 shows that for the second cross-virial coefficient calculations, a total of 489 data of noble gas mixtures taken

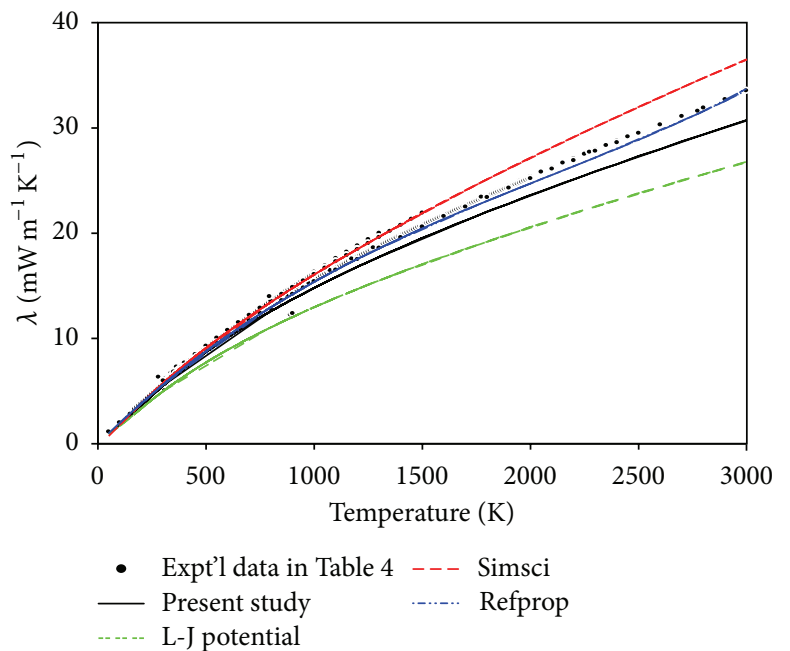

FIGURE 15: Comparison of measured and calculated thermal conductivities for Xe.

from the critical compilation of Dymond et al. [17] and from the Kestin et al. [26] were fitted to be overall average RMSD value of $5.73 \mathrm{~cm}^{3} \mathrm{~mol}^{-1}$, while the $6.25 \mathrm{~cm}^{3} \mathrm{~mol}^{-1}$ value was obtained by the smoothing functions of Dymond et al. in the same fashion as used in the pure gas calculations. And the 45.0 and $9.28 \mathrm{~cm}^{3} \mathrm{~mol}^{-1} \mathrm{RMSD}$ values were found by the original Lennard-Jones (12-6) potential and the 
TABLE 8: Deviations between experimental and predicted thermal conductivities of noble gas mixtures.

\begin{tabular}{|c|c|c|c|c|c|c|}
\hline \multirow{2}{*}{ Mixtures } & \multirow{2}{*}{ Number of points } & \multicolumn{3}{|c|}{ Average $\mathrm{RMSD}_{\mathrm{r}}$ in $\lambda_{\text {mix }}(\%)$} & \multirow{2}{*}{$\Delta T(\mathrm{~K})$} & \multirow{2}{*}{ Reference } \\
\hline & & Present study & L-J & Wassiljewa $^{\mathrm{a}}$ & & \\
\hline \multirow{8}{*}{ Helium-neon } & 32 & 9.69 & 12.2 & 5.98 & $298-973$ & {$[36]$} \\
\hline & 7 & 2.30 & 4.68 & 1.59 & 291 & {$[47]$} \\
\hline & 138 & 5.62 & 8.08 & 3.14 & $275-1175$ & {$[57]$} \\
\hline & 12 & 11.2 & 13.9 & 7.17 & $303-363$ & {$[100]$} \\
\hline & 15 & 8.59 & 11.3 & 3.73 & $350-1050$ & {$[104]$} \\
\hline & 66 & 7.65 & 10.1 & 4.28 & $50-973$ & {$[26]$} \\
\hline & 7 & 12.6 & 12.2 & 6.08 & 298 & [107] \\
\hline & 8 & 7.14 & 7.62 & 6.27 & $302-793$ & {$[110]$} \\
\hline \multirow{11}{*}{ Helium-argon } & 32 & 15.2 & 14.4 & 10.8 & $298-993$ & {$[31]$} \\
\hline & 20 & 8.61 & 9.59 & 12.1 & 291 & {$[47]$} \\
\hline & 4 & 12.9 & 12.2 & 8.31 & 273 & {$[56]$} \\
\hline & 138 & 9.00 & 7.94 & 5.22 & $275-1025$ & {$[57]$} \\
\hline & 15 & 10.8 & 9.46 & 5.98 & $350-1050$ & {$[104]$} \\
\hline & 66 & 8.16 & 7.22 & 3.91 & $50-973$ & {$[26]$} \\
\hline & 9 & 9.20 & 8.23 & 4.99 & 298 & [107] \\
\hline & 12 & 12.3 & 11.8 & 7.54 & $308-363$ & [109] \\
\hline & 8 & 10.6 & 10.5 & 11.6 & $302-793$ & {$[110]$} \\
\hline & 5 & 10.3 & 9.99 & 5.79 & 311 & {$[111]$} \\
\hline & 4 & 9.21 & 8.76 & 6.80 & 273 & [129] \\
\hline \multirow{8}{*}{ Helium-krypton } & 6 & 8.47 & 6.24 & 3.27 & 302 & {$[112]$} \\
\hline & 32 & 9.82 & 6.11 & 4.16 & 298-993 & {$[31]$} \\
\hline & 9 & 5.00 & 3.12 & 1.88 & 291 & {$[57]$} \\
\hline & 15 & 9.92 & 5.27 & 4.18 & $350-1050$ & {$[104]$} \\
\hline & 66 & 8.76 & 5.81 & 3.68 & $50-973$ & {$[26]$} \\
\hline & 9 & 5.39 & 3.09 & 2.29 & 291 & {$[45,46]$} \\
\hline & 16 & 10.1 & 9.50 & 4.52 & $308-363$ & [109] \\
\hline & 12 & 6.23 & 5.49 & 4.16 & $302-793$ & [110] \\
\hline \multirow{7}{*}{ Helium-xenon } & 4 & 3.95 & 8.95 & 1.97 & 302 & [112] \\
\hline & 16 & 4.57 & 7.00 & 3.52 & $303-363$ & {$[100]$} \\
\hline & 15 & 1.54 & 4.05 & 2.62 & $350-1050$ & {$[104]$} \\
\hline & 66 & 7.19 & 8.93 & 4.22 & $50-973$ & {$[26]$} \\
\hline & 10 & 6.19 & 10.02 & 3.61 & 291 & {$[45,46]$} \\
\hline & 8 & 4.17 & 8.2 & 3.43 & $302-793$ & {$[110]$} \\
\hline & 6 & 4.35 & 9.3 & 2.24 & 311 & [111] \\
\hline Average & $888^{*}$ & 8.11 & 8.42 & 4.73 & & \\
\hline \multirow{8}{*}{ Neon-argon } & 32 & 3.82 & 4.91 & 4.91 & $298-973$ & $\begin{array}{l}64] \\
6\end{array}$ \\
\hline & 9 & 3.59 & 5.91 & 5.08 & 291 & {$[47]$} \\
\hline & 12 & 2.87 & 5.15 & 4.36 & $313-363$ & [113] \\
\hline & 24 & 3.51 & 5.70 & 6.54 & $350-1500$ & {$[104]$} \\
\hline & 87 & 2.65 & 4.62 & 5.34 & $50-3273$ & {$[26]$} \\
\hline & 11 & 0.84 & 2.23 & 1.17 & 311 & {$[115]$} \\
\hline & 8 & 0.77 & 2.64 & 2.12 & 298 & {$[107]$} \\
\hline & 8 & 5.22 & 7.33 & 7.34 & $302-793$ & {$[110]$} \\
\hline \multirow{8}{*}{ Neon-krypton } & 32 & 1.37 & 4.94 & 2.10 & $298-973$ & {$[65]$} \\
\hline & 198 & 25.7 & 32.2 & 28.1 & $275-1275$ & {$[57]$} \\
\hline & 12 & 0.97 & 8.51 & 2.26 & $313-363$ & [113] \\
\hline & 24 & 15.8 & 23.6 & 19.2 & $350-1500$ & {$[104]$} \\
\hline & 87 & 3.35 & 7.55 & 2.58 & $50-3273$ & {$[26]$} \\
\hline & 9 & 4.04 & 5.58 & 1.54 & 311 & {$[115]$} \\
\hline & 9 & 3.85 & 5.28 & 2.34 & 291 & {$[45,46]$} \\
\hline & 6 & 6.10 & 10.4 & 6.27 & $302-793$ & [110] \\
\hline
\end{tabular}


TABLE 8: Continued.

\begin{tabular}{|c|c|c|c|c|c|c|}
\hline \multirow{2}{*}{ Mixtures } & \multirow{2}{*}{ Number of points } & \multicolumn{3}{|c|}{ Average $\mathrm{RMSD}_{\mathrm{r}}$ in $\lambda_{\text {mix }}(\%)$} & \multirow{2}{*}{$\Delta T(\mathrm{~K})$} & \multirow{2}{*}{ Reference } \\
\hline & & Present study & L-J & Wassiljewa $^{\mathrm{a}}$ & & \\
\hline \multirow{6}{*}{ Neon-xenon } & 9 & 2.11 & 4.50 & 2.18 & 291 & [57] \\
\hline & 12 & 5.24 & 8.08 & 4.49 & $303-363$ & {$[100]$} \\
\hline & 24 & 4.52 & 7.59 & 5.03 & $350-1500$ & {$[104]$} \\
\hline & 87 & 4.41 & 5.24 & 2.43 & $50-3273$ & {$[26]$} \\
\hline & 9 & 1.90 & 4.42 & 2.04 & 291 & {$[45,46]$} \\
\hline & 6 & 6.00 & 8.72 & 6.06 & $302-793$ & [110] \\
\hline Average & $715^{*}$ & 9.94 & 13.7 & 11.0 & & \\
\hline \multirow{8}{*}{ Argon-krypton } & 32 & 1.14 & 10.3 & 2.42 & $298-973$ & {$[64]$} \\
\hline & 9 & 5.65 & 5.02 & 3.31 & 291 & [57] \\
\hline & 24 & 2.11 & 9.69 & 2.51 & $350-1500$ & {$[104]$} \\
\hline & 87 & 2.73 & 10.1 & 3.32 & $50-3273$ & {$[26]$} \\
\hline & 6 & 4.70 & 5.58 & 2.46 & 311 & [115] \\
\hline & 8 & 5.77 & 5.28 & 3.58 & 291 & {$[45,46]$} \\
\hline & 12 & 3.52 & 7.30 & 1.50 & $308-363$ & [109] \\
\hline & 6 & 3.32 & 13.2 & 5.55 & $302-793$ & {$[110]$} \\
\hline \multirow{7}{*}{ Argon-xenon } & 9 & 2.63 & 5.59 & 2.94 & 291 & {$[57]$} \\
\hline & 8 & 10.5 & 17.1 & 10.0 & $311-366$ & [113] \\
\hline & 24 & 1.14 & 8.46 & 1.38 & $350-1500$ & [104] \\
\hline & 87 & 3.10 & 8.11 & 2.34 & $50-3273$ & {$[26]$} \\
\hline & 9 & 2.87 & 5.43 & 3.19 & 291 & {$[45,46]$} \\
\hline & 6 & 3.43 & 10.8 & 3.14 & $302-793$ & {$[110]$} \\
\hline & 6 & 4.05 & 4.03 & 4.94 & 311 & [111] \\
\hline Average & $333^{*}$ & 2.97 & 8.90 & 2.92 & & \\
\hline \multirow{7}{*}{ Krypton-xenon } & 5 & 4.42 & 14.9 & 3.91 & 302 & {$[112]$} \\
\hline & 9 & 1.87 & 12.5 & 1.03 & 291 & {$[57]$} \\
\hline & 12 & 7.14 & 18.3 & 7.24 & $313-363$ & [113] \\
\hline & 24 & 2.35 & 13.7 & 2.37 & $350-1500$ & {$[104]$} \\
\hline & 87 & 5.13 & 16.0 & 3.97 & $50-3273$ & {$[26]$} \\
\hline & 9 & 1.76 & 12.4 & 0.92 & 291 & {$[45,46]$} \\
\hline & 10 & 6.06 & 17.5 & 5.90 & $302-793$ & {$[110]$} \\
\hline Average & $156^{*}$ & 4.51 & 15.5 & 3.75 & & \\
\hline Overall & $2092^{* *}$ & 7.65 & 10.8 & 6.50 & & \\
\hline
\end{tabular}

${ }^{*}$ Number of data points for each mixture.

** Total number of data points for all gas mixture mixtures.

${ }^{a}$ Wassiljewa equation [15] with the combinational factor of Mason and Saxena [16].

corresponding states method of Tsonopoulos, respectively. A comparison of measured and calculated second cross-virial coefficients of $\mathrm{Ar}+\mathrm{Kr}$ mixture is shown in Figure 21.

Prediction results of noble gas mixture viscosities are presented in Table 7. A total of 2918 viscosity data points for all noble gas mixtures were calculated in this paper, indicating that the present study is in better agreement between experimental and calculated data than other methods on a $\% \mathrm{RMSD}_{\mathrm{r}}$ criterion: $2.93 \%$ by the present model, $10.6 \%$ by the original Lennard-Jones (12-6) potential, and $4.05 \%$ by the Lucas method. However, it is noted that for krypton and xenon mixture, this work is less reliable to the Lucas method. Figure 22 shows the comparison of measured and calculated viscosities of $\mathrm{He}+\mathrm{Ne}$ mixture.

Given in Table 8 are the resulting $\% \mathrm{RMSD}_{\mathrm{r}}$ values between a total of 2092 measured and predicted mixture thermal conductivity data. Based on the overall average $\% \mathrm{RMSD}_{\mathrm{r}}$ value of all noble gas mixtures, $7.65 \%$ of the present study is in slightly worse agreement between measured and predicted mixture thermal conductivities than $6.50 \%$ of the Wassiljewa equation [15] with the combinational factor of Mason and Saxena [16] and is more feasible to $10.8 \%$ of the original Lennard-Jones (12-6) potential. A comparison of measured and calculated thermal conductivities of $\mathrm{Ne}+\mathrm{Kr}$ mixture is shown in Figure 23.

Included in Table 9 are the resulting $\% \mathrm{RMSD}_{\mathrm{r}}$ values between a total of 1240 measured and predicted binary diffusion coefficient data for noble gas mixtures, in which $4.98 \% \mathrm{RMSD}_{\mathrm{r}}$ of the present study is in quite better agreement between measured and predicted binary diffusion coefficients than $7.95 \% \mathrm{RMSD}_{\mathrm{r}}$ of the original Lennard-Jones (12-6) potential and 6.24 \%RMSD $\mathrm{R}_{\mathrm{r}}$ of the Fuller method. 
TABLE 9: Deviations between experimental and predicted binary diffusion coefficients of noble gas mixtures.

\begin{tabular}{|c|c|c|c|c|c|c|}
\hline \multirow{2}{*}{ Compound } & \multirow{2}{*}{ Number of points } & \multicolumn{3}{|c|}{ Average $\mathrm{RMSD}_{\mathrm{r}}$ in $D(\%)$} & \multirow[b]{2}{*}{$\Delta T(\mathrm{~K})$} & \multirow[b]{2}{*}{ Reference } \\
\hline & & Present study & L-J & Fuller & & \\
\hline \multirow{9}{*}{ Helium-neon } & 26 & 2.45 & 3.77 & 10.6 & $50-1773$ & {$[26]$} \\
\hline & 7 & 1.04 & 3.09 & 8.31 & $200-673$ & {$[29]$} \\
\hline & 1 & 5.20 & 1.32 & 12.7 & 1000 & [130] \\
\hline & 5 & 2.01 & 6.19 & 3.52 & $65-295$ & {$[131]$} \\
\hline & 6 & 3.52 & 1.03 & 9.42 & $298-973$ & {$[36]$} \\
\hline & 26 & 9.89 & 7.35 & 5.09 & $200-1250$ & {$[132]$} \\
\hline & 20 & 5.27 & 4.93 & 12.7 & $297-1268$ & {$[121]$} \\
\hline & 1 & 14.0 & 10.6 & 8.99 & 293 & {$[133]$} \\
\hline & 14 & 2.81 & 5.29 & 7.79 & $273-683$ & {$[56]$} \\
\hline \multirow{20}{*}{ Helium-argon } & 29 & 2.35 & 2.72 & 7.04 & $50-3273$ & {$[26]$} \\
\hline & 7 & 0.56 & 0.12 & 4.91 & $200-673$ & [29] \\
\hline & 1 & 8.91 & 8.18 & 0.77 & 1000 & {$[130]$} \\
\hline & 3 & 3.15 & 4.11 & 2.93 & $276-346$ & {$[134]$} \\
\hline & 8 & 2.78 & 3.57 & 4.06 & $298-498$ & {$[135]$} \\
\hline & 1 & 1.66 & 2.67 & 0.67 & 298 & {$[136]$} \\
\hline & 1 & 0.91 & 0.08 & 1.87 & 298 & [137] \\
\hline & 3 & 3.84 & 3.71 & 2.87 & $287-418$ & {$[138]$} \\
\hline & 6 & 8.30 & 7.80 & 1.68 & $298-1100$ & [139] \\
\hline & 1 & 1.21 & 2.22 & 0.17 & 296 & {$[140]$} \\
\hline & 1 & 0.58 & 1.63 & 1.11 & 273 & {$[12]$} \\
\hline & 1 & 1.69 & 2.75 & 0.01 & 273 & {$[55]$} \\
\hline & 2 & 5.42 & 4.92 & 4.23 & $276-418$ & {$[18]$} \\
\hline & 4 & 1.37 & 2.84 & 2.76 & $90-400$ & {$[131]$} \\
\hline & 14 & 2.63 & 2.50 & 2.65 & $251-418$ & {$[141]$} \\
\hline & 17 & 5.01 & 5.08 & 3.39 & $276-1100$ & {$[142]$} \\
\hline & 8 & 5.69 & 5.05 & 0.65 & 298-993 & {$[31]$} \\
\hline & 26 & 6.02 & 5.59 & 2.13 & $200-1250$ & {$[132]$} \\
\hline & 21 & 9.66 & 8.79 & 6.62 & $298-1272$ & {$[121]$} \\
\hline & 84 & 8.65 & 8.68 & 5.25 & $118-4500$ & {$[56]$} \\
\hline \multirow{8}{*}{ Helium-krypton } & 29 & 2.60 & 7.00 & 5.51 & $50-3273$ & {$[26]$} \\
\hline & 7 & 2.44 & 5.18 & 6.12 & $200-673$ & {$[29]$} \\
\hline & 1 & 0.88 & 8.40 & 0.34 & 1000 & {$[130]$} \\
\hline & 4 & 14.2 & 17.0 & 18.6 & $111-400$ & {$[131]$} \\
\hline & 8 & 2.04 & 7.40 & 4.22 & 298-993 & {$[31]$} \\
\hline & 26 & 5.12 & 2.86 & 3.41 & 200-940 & [132] \\
\hline & 21 & 5.32 & 12.4 & 8.11 & $307-1274$ & {$[121]$} \\
\hline & 4 & 3.48 & 4.02 & 6.69 & $273-318$ & {$[56]$} \\
\hline \multirow{10}{*}{ Helium-xenon } & 29 & 7.21 & 13.1 & 5.35 & $50-3273$ & {$[26]$} \\
\hline & 7 & 5.13 & 11.8 & 4.80 & $200-673$ & [29] \\
\hline & 1 & 11.0 & 17.5 & 2.85 & 1000 & [130] \\
\hline & 4 & 6.21 & 12.6 & 6.93 & $169-400$ & {$[131]$} \\
\hline & 6 & 1.69 & 8.48 & 3.59 & $298-778$ & {$[38]$} \\
\hline & 26 & 9.4 & 15.6 & 4.93 & 200-940 & {$[132]$} \\
\hline & 21 & 9.96 & 16.3 & 6.65 & $297-1270$ & {$[121]$} \\
\hline & 8 & 7.6 & 13.5 & 8.01 & 273-394 & {$[56]$} \\
\hline & 10 & 2.2 & 8.97 & 2.82 & $220-400$ & [143] \\
\hline & 2 & 2.3 & 9.1 & 2.97 & $273-315$ & [144] \\
\hline Average & $557^{*}$ & 5.80 & 7.61 & 5.72 & & \\
\hline
\end{tabular}


TABle 9: Continued.

\begin{tabular}{|c|c|c|c|c|c|c|}
\hline \multirow{2}{*}{ Compound } & \multirow{2}{*}{ Number of points } & \multicolumn{3}{|c|}{ Average $\mathrm{RMSD}_{\mathrm{r}}$ in $D(\%)$} & \multirow[b]{2}{*}{$\Delta T(\mathrm{~K})$} & \multirow[b]{2}{*}{ Reference } \\
\hline & & Present study & L-J & Fuller & & \\
\hline \multirow{12}{*}{ Neon-argon } & 29 & 2.03 & 3.08 & 5.38 & $50-3273$ & {$[26]$} \\
\hline & 7 & 1.64 & 2.83 & 2.25 & $200-673$ & {$[29]$} \\
\hline & 1 & 3.38 & 6.84 & 1.17 & 1000 & {$[130]$} \\
\hline & 4 & 2.60 & 3.14 & 3.36 & $90-400$ & {$[131]$} \\
\hline & 8 & 0.76 & 3.61 & 2.50 & $298-973$ & {$[64]$} \\
\hline & 5 & 5.42 & 1.66 & 4.03 & $90-473$ & {$[145]$} \\
\hline & 4 & 2.77 & 1.72 & 2.10 & $273-318$ & {$[146]$} \\
\hline & 26 & 1.86 & 3.76 & 1.51 & $200-940$ & {$[132]$} \\
\hline & 20 & 5.89 & 7.29 & 4.91 & $297-1274$ & {$[121]$} \\
\hline & 1 & 3.21 & 7.39 & 7.77 & 293 & {$[147,148]$} \\
\hline & 19 & 3.15 & 3.25 & 3.52 & $194-680$ & {$[56]$} \\
\hline & 5 & 5.47 & 0.79 & 1.06 & $277-365$ & [149] \\
\hline \multirow{9}{*}{ Neon-krypton } & 29 & 2.06 & 10.4 & 6.99 & $50-3273$ & {$[26]$} \\
\hline & 7 & 2.31 & 10.4 & 8.25 & $200-673$ & [29] \\
\hline & 1 & 0.58 & 12.3 & 4.19 & 1000 & {$[130]$} \\
\hline & 4 & 4.37 & 9.32 & 6.83 & $111-400$ & {$[131]$} \\
\hline & 4 & 6.11 & 7.25 & 6.32 & $273-318$ & [146] \\
\hline & 8 & 2.75 & 9.80 & 5.78 & 298-973 & {$[65]$} \\
\hline & 26 & 3.73 & 13.0 & 8.44 & $200-940$ & {$[132]$} \\
\hline & 20 & 5.72 & 9.45 & 5.31 & $269-1270$ & {$[121]$} \\
\hline & 4 & 6.11 & 7.25 & 6.32 & $273-318$ & {$[56]$} \\
\hline \multirow{9}{*}{ Neon-xenon } & 29 & 5.49 & 11.8 & 6.50 & $50-3273$ & {$[26]$} \\
\hline & 7 & 4.82 & 11.9 & 7.49 & $200-673$ & [29] \\
\hline & 1 & 6.22 & 13.3 & 3.79 & 1000 & [130] \\
\hline & 4 & 4.09 & 11.1 & 6.82 & $169-400$ & {$[131]$} \\
\hline & 6 & 2.03 & 9.38 & 4.24 & $298-773$ & {$[38]$} \\
\hline & 26 & 6.97 & 7.81 & 7.58 & $200-940$ & {$[132]$} \\
\hline & 21 & 8.96 & 15.5 & 9.15 & $297-1270$ & {$[121]$} \\
\hline & 4 & 1.14 & 8.37 & 4.82 & $273-318$ & {$[56]$} \\
\hline & 5 & 5.59 & 12.6 & 9.16 & $275-362$ & [149] \\
\hline Average & $335^{*}$ & 4.15 & 8.18 & 5.73 & & \\
\hline \multirow{14}{*}{ Argon-krypton } & 29 & 3.49 & 7.91 & 10.0 & $50-3273$ & {$[26]$} \\
\hline & 7 & 2.37 & 6.02 & 4.28 & $200-673$ & [29] \\
\hline & 1 & 0.08 & 8.05 & 0.37 & 1000 & {$[130]$} \\
\hline & 1 & 0.33 & 7.03 & 0.26 & 273 & {$[18]$} \\
\hline & 4 & 2.26 & 4.42 & 5.99 & $169-400$ & {$[131]$} \\
\hline & 8 & 8.60 & 5.25 & 19.2 & $77-600$ & {$[150]$} \\
\hline & 9 & 4.01 & 7.89 & 3.39 & $199-473$ & {$[142]$} \\
\hline & 5 & 5.85 & 8.91 & 5.25 & $273-473$ & [145] \\
\hline & 4 & 3.85 & 3.69 & 2.98 & $273-318$ & {$[146]$} \\
\hline & 8 & 1.87 & 8.82 & 3.42 & $298-973$ & {$[64]$} \\
\hline & 11 & 2.23 & 4.79 & 2.89 & $200-400$ & {$[151]$} \\
\hline & 26 & 9.30 & 15.3 & 10.6 & $200-940$ & {$[132]$} \\
\hline & 21 & 2.88 & 9.59 & 4.20 & $300-1274$ & {$[121]$} \\
\hline & 13 & 3.51 & 7.89 & 3.37 & $199-407$ & {$[56]$} \\
\hline \multirow{3}{*}{ Argon-xenon } & 29 & 5.48 & 8.62 & 11.5 & $50-3273$ & {$[26]$} \\
\hline & 7 & 2.46 & 6.39 & 6.68 & $200-673$ & {$[29]$} \\
\hline & 1 & 7.99 & 13.0 & 3.23 & 1000 & {$[130]$} \\
\hline
\end{tabular}


TABLe 9: Continued.

\begin{tabular}{|c|c|c|c|c|c|c|}
\hline \multirow{2}{*}{ Compound } & \multirow{2}{*}{ Number of points } & \multicolumn{3}{|c|}{ Average $\mathrm{RMSD}_{\mathrm{r}}$ in $D(\%)$} & \multirow[b]{2}{*}{$\Delta T(\mathrm{~K})$} & \multirow[b]{2}{*}{ Reference } \\
\hline & & Present study & L-J & Fuller & & \\
\hline & 2 & 5.72 & 6.25 & 6.34 & $195-378$ & {$[18]$} \\
\hline & 4 & 2.43 & 5.43 & 10.6 & $169-400$ & {$[131]$} \\
\hline & 4 & 3.16 & 6.25 & 6.88 & $194-378$ & {$[152]$} \\
\hline & 6 & 1.86 & 6.41 & 3.16 & $298-773$ & {$[38]$} \\
\hline & 26 & 5.57 & 9.95 & 4.59 & $200-940$ & [132] \\
\hline & 21 & 4.18 & 7.51 & 5.35 & $298-1272$ & [121] \\
\hline & 19 & 3.79 & 7.11 & 9.07 & $173-1597$ & [153] \\
\hline & 13 & 3.93 & 6.96 & 5.83 & 194-394 & {$[56]$} \\
\hline Average & $279^{*}$ & 4.48 & 8.42 & 7.16 & & \\
\hline \multirow{6}{*}{ Krypton-xenon } & 29 & 4.46 & 7.05 & 12.1 & $50-3273$ & {$[26]$} \\
\hline & 7 & 1.38 & 4.42 & 7.54 & $200-673$ & [29] \\
\hline & 1 & 4.70 & 10.5 & 4.63 & 1000 & [130] \\
\hline & 4 & 1.38 & 3.27 & 12.2 & $169-400$ & [131] \\
\hline & 6 & 1.10 & 4.72 & 2.88 & $298-773$ & [38] \\
\hline & 22 & 7.00 & 11.1 & 7.52 & $297-1270$ & [121] \\
\hline Average & $69^{*}$ & 4.49 & 7.70 & 9.27 & & \\
\hline Overall & $1240^{* *}$ & 4.98 & 7.95 & 6.24 & & \\
\hline
\end{tabular}

${ }^{*}$ Number of data points for each mixture. ${ }^{* *}$ Total number of data points for all gas mixtures.

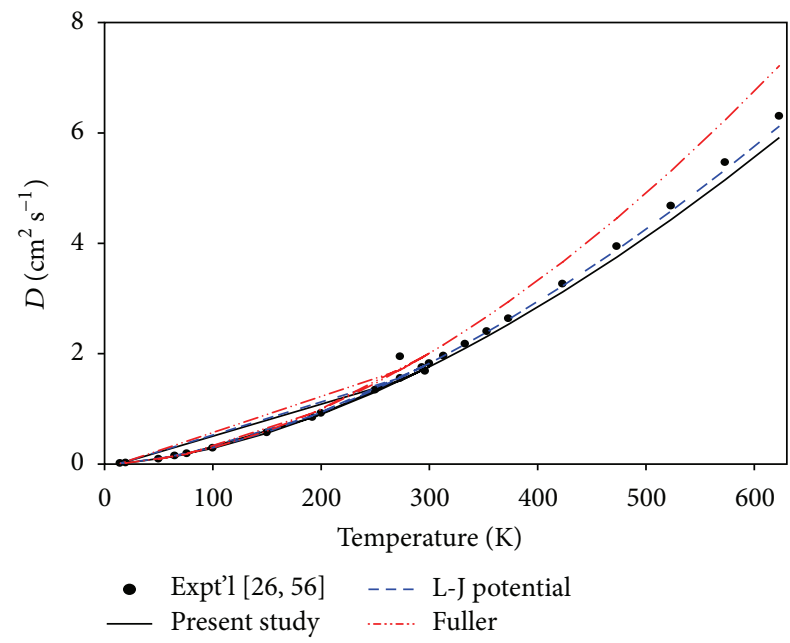

FIGURE 16: Comparison of measured and calculated self-diffusion coefficients for He.

A comparison of measured and calculated binary diffusion coefficients for $\mathrm{He}+\mathrm{Ne}$ mixture is shown in Figure 24 .

\section{Conclusions}

The three-parameter Lennard-Jones (12-6) potential function has been empirically modified by introducing a temperature-correction parameter to the reduced temperature $T^{*}$ for the calculation of the thermodynamic property (second virial coefficient) and dilute transport properties (viscosity, thermal conductivity, and diffusion coefficient) of noble gases ( $\mathrm{He}, \mathrm{Ne}, \mathrm{Ar}, \mathrm{Kr}$, and $\mathrm{Xe}$ ) and their binary mixtures. Separately for each species, a single set of three

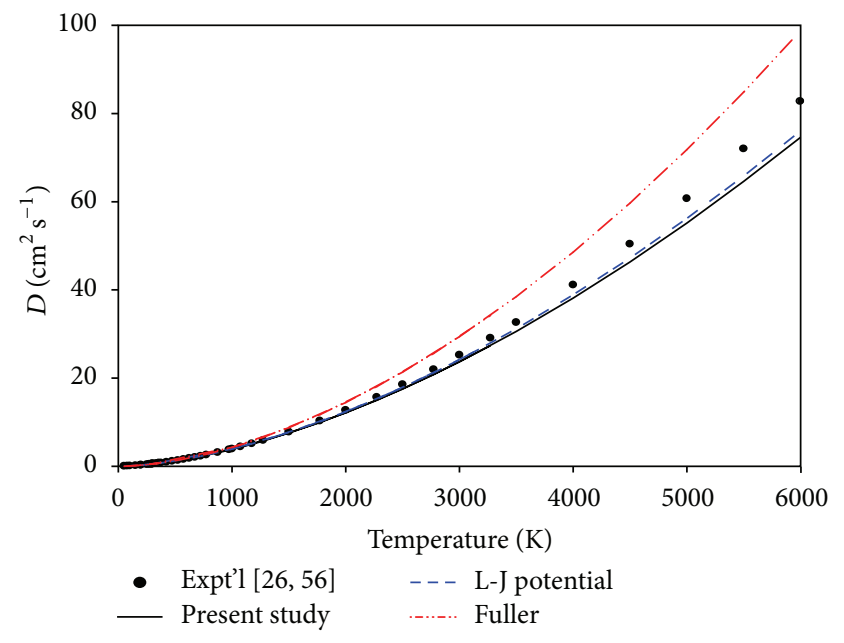

FIGURE 17: Comparison of measured and calculated self-diffusion coefficients for $\mathrm{Ne}$.

potential parameters $(\sigma, \varepsilon$, and $\tau)$ is estimated when the second virial coefficient and viscosity data are regressed together within the experimental errors. Obtained potential parameters are used to reproduce second virial coefficient and viscosity data and in all following predictions of other properties like thermal conductivity and diffusion coefficient. Noble gas mixture properties are calculated with the same set of parameters as well.

For the second virial coefficient calculations of pure noble gases, the three-parameter Lennard-Jones (12-6) potential proposed in this paper is quite comparable to Dymond's correlations and produces more accurate results than the original two-parameter Lennard-Jones (12-6) potential, the 


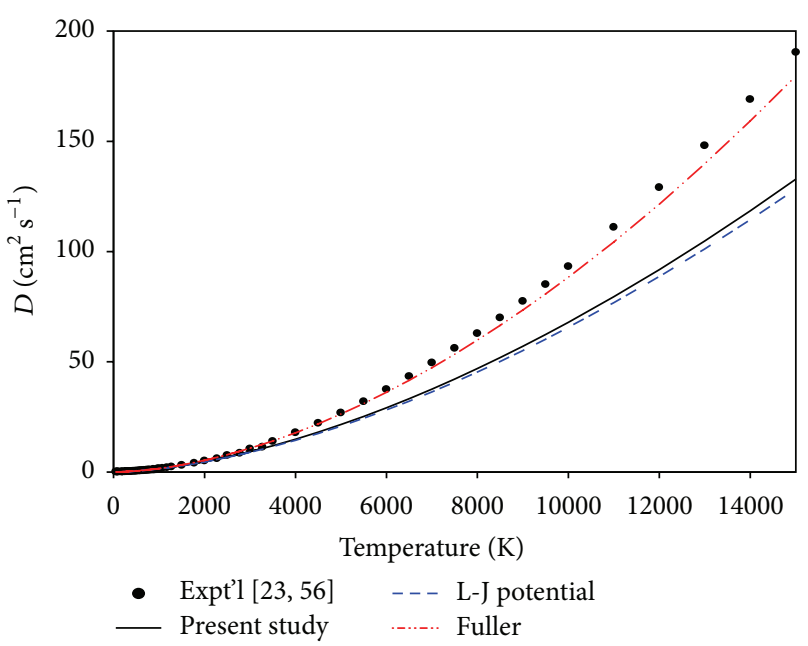

Figure 18: Comparison of measured and calculated self-diffusion coefficients for Ar.

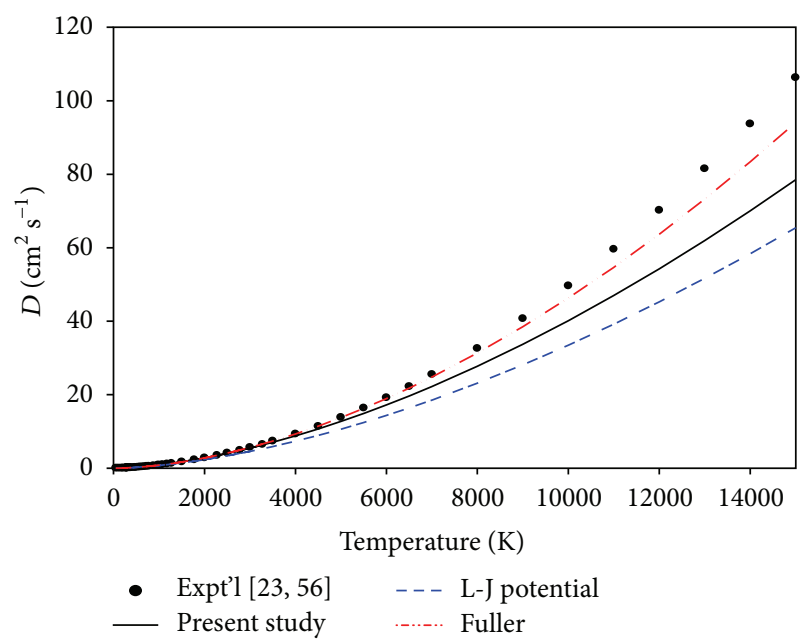

FIGURE 19: Comparison of measured and calculated self-diffusion coefficients for Kr.

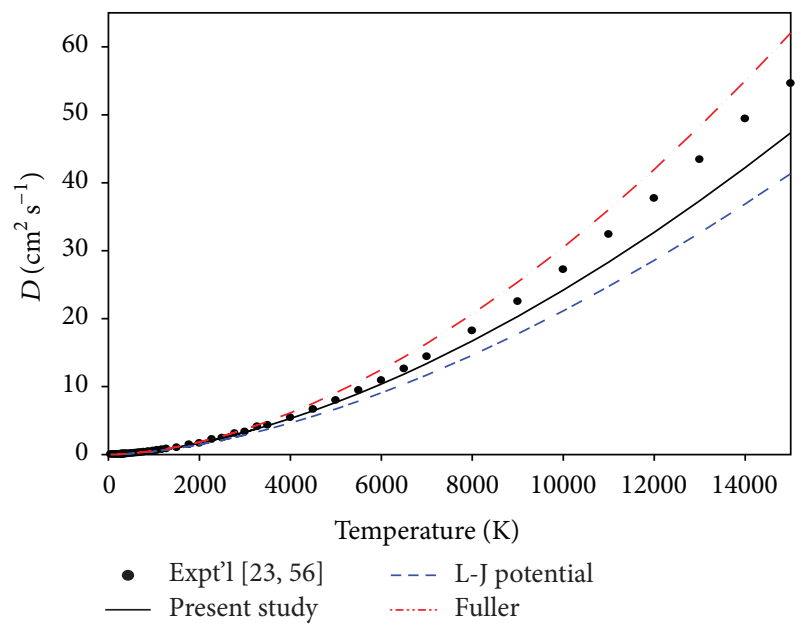

FIGURE 20: Comparison of measured and calculated self-diffusion coefficients for Xe.

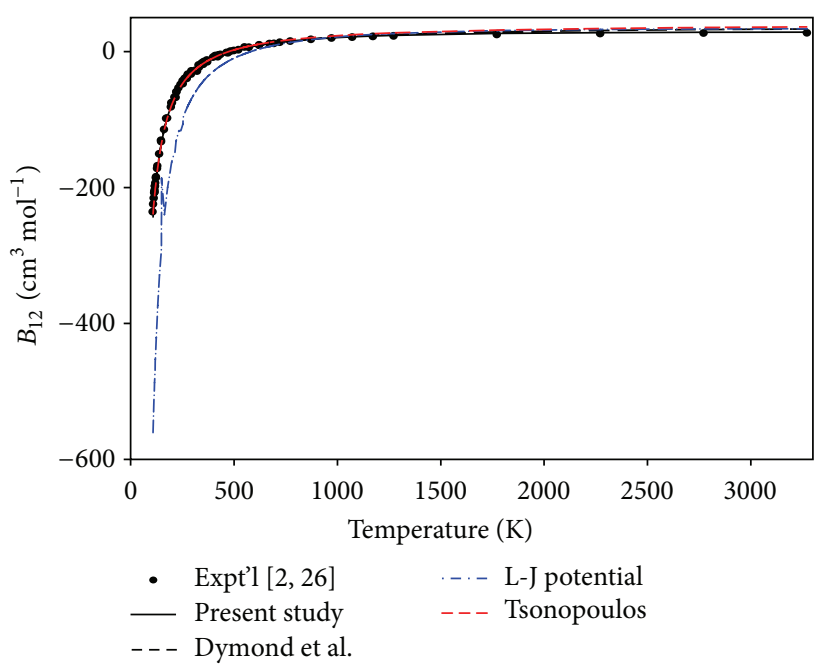

FIGURE 21: Comparison of measured and calculated second crossvirial coefficients for $\mathrm{Ar}+\mathrm{Kr}$ mixture.
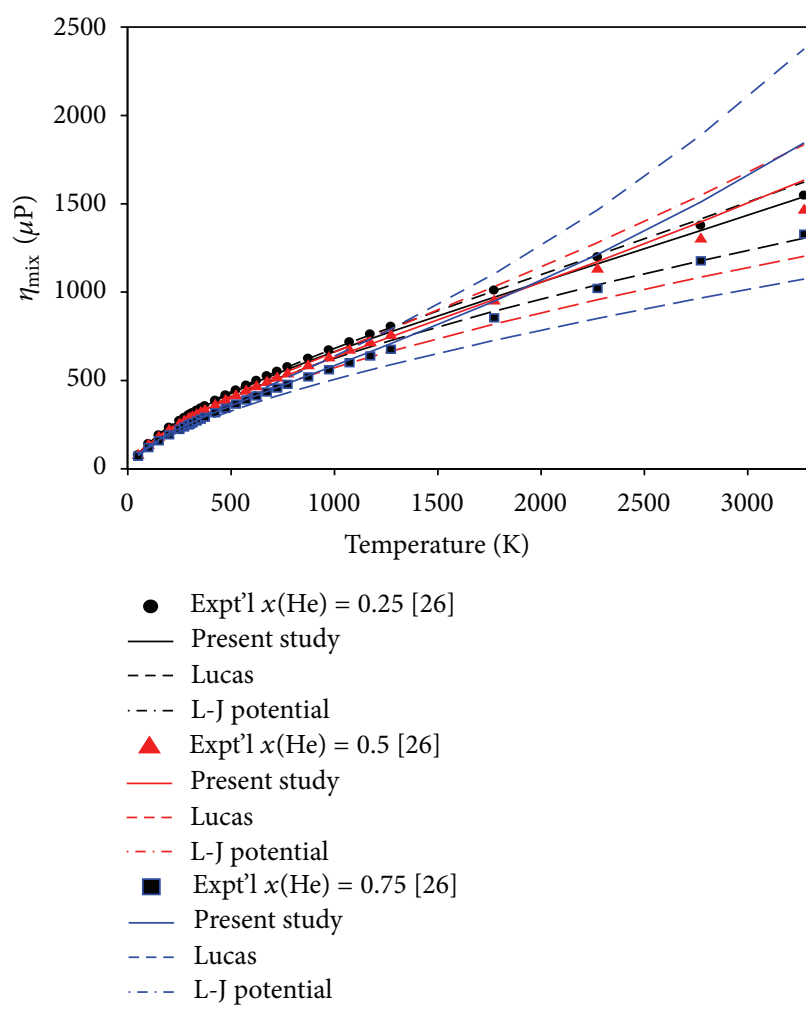

FIGURE 22: Comparison of measured and calculated viscosities for $\mathrm{He}+\mathrm{Ne}$ mixture

Kihara potential with group contribution concept, and the Tsonopoulos correlations. For the viscosity calculations, the proposed model agrees better with the observed and calculated data than the original Lennard-Jones (12-6) potential, the Kihara potential with group contribution method, the Lucas method, the Simsci Database, and the Refprop Database. Agreement between experimental and calculated thermal conductivities obtained by the proposed model 


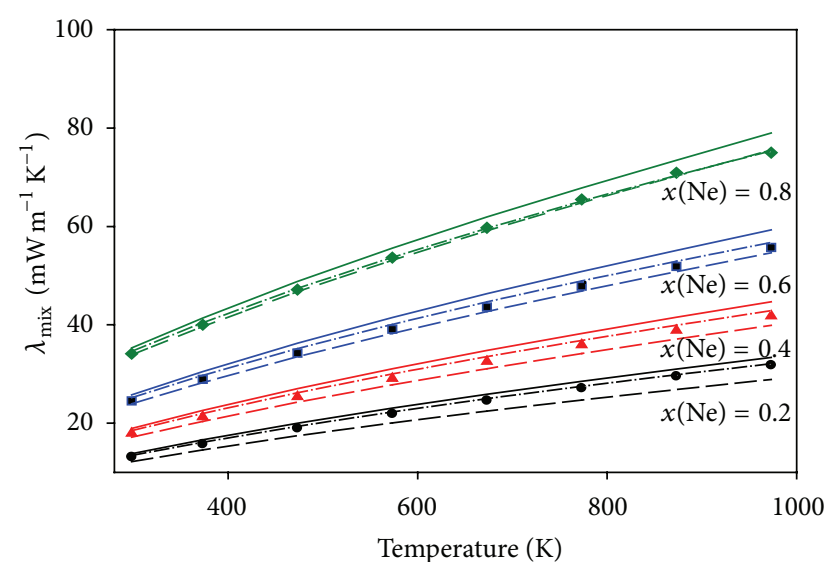

- Expt'l [26]

— Present study

--- L-J potential

...- Wassiljewa

- Expt'l [26]

— Present study

- - - L-J potential

-.- Wassiljewa

- Expt'1 [26]

— Present study

-- - L-J potential

-... Wassiljewa

- Expt'l[26]

— Present study

-- L-J potential

-..- Wassiljewa

FIGURE 23: Comparison of measured and calculated thermal conductivities for $\mathrm{Ne}+\mathrm{Kr}$ mixture.

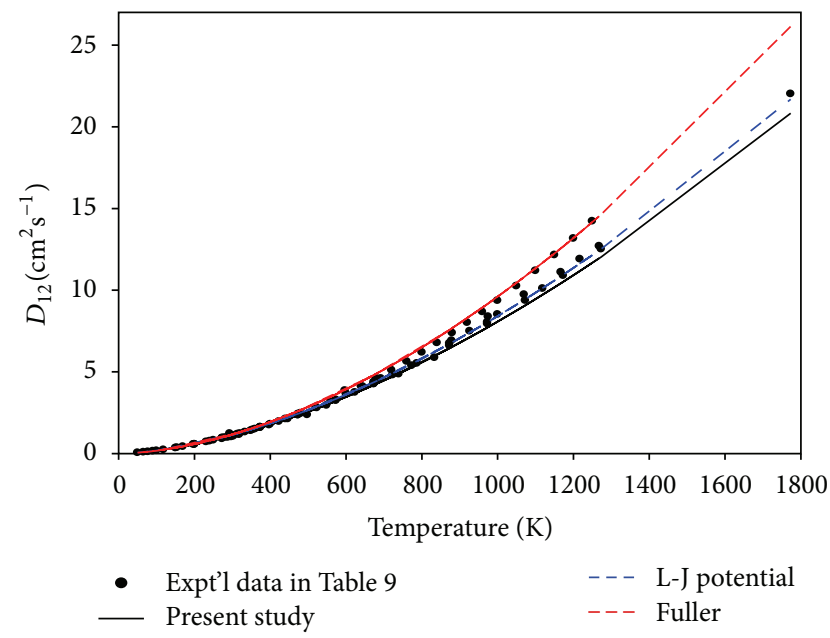

FIGURE 24: Comparison of measured and calculated binary diffusion coefficients for $\mathrm{He}+\mathrm{Ne}$ mixture.

is somewhat less accurate than the Simsci Database, but compares very well with the original Lennard-Jones (12-6) potential and with the Refprop Database. Calculation of self-diffusion coefficients shows that this work is in better agreement with experimental data than those of the original Lennard-Jones (12-6) potential and of the Fuller method, and that for helium gas and for neon gas results of the proposed method is less accurate than the original LennardJones (12-6) potential.

For mixture property predictions, the same set of potential parameters is applied with no additional parameters. Second cross-virial coefficient data calculated by the present study is less feasible to those of Dymond's correlations and is in better agreement with the observed data than the original Lennard-Jones (12-6) potential and the corresponding states method of Tsonopoulos. The present study is in better agreement between experimental mixture viscosity data than the original Lennard-Jones (12-6) potential and the Lucas method, except for the mixture of $\mathrm{kr}+\mathrm{Xe}$. The present study is in somewhat worse agreement between measured and calculated mixture thermal conductivities than the Wassiljewa equation with the combinational factor of Mason and Saxena and is more accurate than the original Lennard-Jones (12-6) potential. However, thermal conductivity of the $\mathrm{He}+\mathrm{Ne}$ and $\mathrm{Kr}+\mathrm{Xe}$ mixtures was reproduced better with the Wassiljewa equation than with the proposed method. The present study is in appreciably better agreement between the observed and calculated binary diffusion coefficients of noble gases mixtures than the original Lennard-Jones (12-6) potential and the Fuller method.

In this work, the empirical approach of adding a temperature-correction parameter to the reduced temperature in the Lennard-Jones (12-6) potential function has been tested with good success for the calculations of thermodynamic and transport property of noble gases and their binary mixtures in dilute gas region. Application of this approach to other substances such as polyatomic, polar gases will be tested in the near future.

\section{Nomenclature}

A: $\quad$ Combinational factor in (18)

$A^{*}: \quad$ Defined by $(13), \Omega^{(2,2) *}\left(T^{*}\right) / \Omega^{(1,1) *}\left(T^{*}\right)$

B: $\quad$ Second virial coefficient $\left(\mathrm{cm}^{3} \mathrm{~mol}^{-1}\right)$

D: $\quad$ Diffusion coefficient $\left(\mathrm{cm}^{2} \mathrm{sec}^{-1}\right)$

$k_{B}$ : Boltzmann constant,

$1.3806488 \cdot 10^{-23}\left(\mathrm{JK}^{-1}\right)$

M: $\quad$ Molecular weight $\left(\mathrm{gram} \mathrm{mol}^{-1}\right)$

$N_{A}: \quad$ Avogadro constant, $6.022 \cdot 10^{23}\left(\mathrm{~mol}^{-1}\right)$

$P: \quad$ Pressure (bar)

$R: \quad$ Distance between molecular centers of molecules 1 and 2 (Â)

$\mathrm{RMSD}_{\mathrm{r}}$ : $\quad$ Root-mean-square deviation $\left(\mathrm{cm}^{3} \mathrm{~mol}^{-1}\right)$

$\% \mathrm{RMSD}_{\mathrm{r}}$ : Percent relative root-mean-square deviation, relative (\%)

T: $\quad$ Absolute temperature $(\mathrm{K})$

$T^{*}: \quad$ Reduced temperature, $k_{B}(T-\tau) / \varepsilon$

$U: \quad$ Intermolecular potential function

$y: \quad$ Mole fraction in the gas phase

$X_{\eta}: \quad$ Defined by (10)

$Y_{\eta}: \quad$ Defined by (11)

$Z_{\eta}: \quad$ Defined by (12).

\section{Greek Letters}

$\varepsilon$ : Depth of the potential well [J]

$\eta$ : Viscosity $(\mu \mathrm{P})$

$\lambda$ : Thermal conductivity $\left(\mathrm{mW} \mathrm{m}^{-1} \mathrm{~K}^{-1}\right)$

$\sigma$ : Collision diameter (Á)

$\tau$ : Reduced temperature-correction parameter 
$\Omega^{(1,1) *}$ : Collision integral for diffusion coefficient

$\Omega^{(2,2) *}$ : Collision integral for viscosity.

\section{Acknowledgment}

This paper was supported by the Konyang University Research Fund, in 2012-2013.

\section{References}

[1] T. H. Chung, M. Ajlan, L. L. Lee, and K. E. Starling, "Generalized multiparameter correlation for nonpolar and polar fluid transport properties," Industrial \& Engineering Chemistry Research, vol. 27, no. 4, pp. 671-679, 1988.

[2] J. P. O'Connell and J. M. Prausnitz, "Advances in thermophysical properties at extreme temperatures and pressures," in Proceedings of the 3rd Symposium of Thermophysical Properties, ASME, New York, NY, USA, 1965.

[3] S. Chapman and T. G. Cowling, The Mathematical Theory of Non-Uniform Gases, Cambridge University Press, New York, NY, USA, 3rd edition, 1970.

[4] J. O. Hirschfelder, C. F. Curtiss, and R. B. Bird, Molecular Theory of Gases and Liquids, Wiley, New York, NY, USA, 1954.

[5] J. H. Dymond and B. J. Alder, "Pair potential for Argon," The Journal of Chemical Physics, vol. 51, no. 50, pp. 309-320, 1969.

[6] L. S. Tee, S. Gotoh, and W. E. Stewart, "Molecular parameters for normal fluids. Lennard-Jones 12-6 Potential," Industrial and Engineering Chemistry Fundamentals, vol. 5, no. 3, pp. 356-363, 1966.

[7] L. S. Tee, S. Gotoh, and W. E. Stewart, "molecular parameters for normal fluids. kihara potential with spherical core," Industrial and Engineering Chemistry Fundamentals, vol. 5, no. 3, pp. 363367, 1966.

[8] J. E. Lennard-Jones, "On the determination of molecular fields. II. from the equation of state of a gas," Proceedings of the Royal Society A, vol. 106, no. 738, pp. 463-477, 1924.

[9] T. Kojima, A Study on measurement for speed of sound in R125, $R 143 a$, and the mixture and precise determination of thermodynamic properties base on intermolecular potential model [M.S. thesis], Keio University, Yokohama, Japan, 2001.

[10] K. Ichikura, Y. Kano, and H. Sato, "Importance of third virial coefficients for representing the gaseous phase based on measuring PVT-properties of 1,1,1-Trifluoroethane (R143a)," International Journal of Thermophysics, vol. 27, pp. 23-38, 2006.

[11] B. E. Poling, J. M. Prausnitz, and J. P. O'Connell, The Properties of Gases and Liquids, McGraw Hill, New York, NY, USA, 5th edition, 2004.

[12] P. D. Neufeld, A. R. Janzen, and R. A. Aziz, "Empirical equations to calculate 16 of the transport collision integrals $\Omega^{(l, s) *}$ for the lennard-jones (12-6) potential," The Journal of Chemical Physics, vol. 57, no. 3, pp. 1100-1102, 1972.

[13] R. Brokaw, "Predicting transport properties of dilute gases," Industrial \& Engineering Chemistry Process Design and Development, vol. 8, no. 2, pp. 240-253, 1969.

[14] H. J. M. Hanley and M. Klein, "Application of the m-6-8 potential to simple gases," The Journal of Physical Chemistry, vol. 76, no. 12, pp. 1743-1751, 1972.

[15] A. Wassiljewa, "Wärmeleitung in Gasgemischen," Physikalische Zeitschrift, vol. 5, p. 737, 1904.
[16] E. A. Mason and S. C. Saxena, "Approximate formula for the thermal conductivity of gas mixtures," Physics of Fluids, vol. 1, no. 5, pp. 361-369, 1958.

[17] J. H. Dymond, K. N. Marsh, R. C. Wilhoit, and K. C. Wong, Virial Coefficients of Pure Gases and Mixtures, vol. 4 of LandoltBörnstein: Numerical Data and Functional Relationships in Science and Technology, part 21A\&B, Springer, Hidelberg, Germany, 2002.

[18] K. Stephan and K. Lucas, Viscosity of Dense Fluids, Plenum Press, New York, NY, USA, 1979.

[19] IMSL, IMSL STAT/LIBRARY: Regression, IMSL, Houston, Tex, USA, 1994.

[20] S.-K. Oh, "An extension of the group contribution method for estimating thermodynamic and transport properties. Part III. Noble gases," Korean Journal of Chemical Engineering, vol. 22, no. 6, pp. 949-959, 2005.

[21] C. Tsonopoulos, "Empirical correlation of second virial coefficients," AIChE Journal, vol. 20, no. 2, pp. 263-272, 1974.

[22] E. W. Lemmon, M. L. Huber, and M. O. McLinden, "NIST standard reference database 23," Reference Fluid Thermodynamic and Transport Properties (REFPROP), version 9.0, National Institute of Standards and Technology, 2010.

[23] K. Lucas, Phase Equilibria and Fluid Properties in the Chemical Industry, Dechema, Frankfurt, Germany, 1980.

[24] Simsci Database, Pro/II with Provision Software, http://iom invensys.com/EN/Pages/SimSci-Esscor_ProcessEngSuite_PROII .aspx.

[25] E. N. Fuller, P. D. Schettler, and J. C. Giddings, "new method for prediction of binary gas-phase diffusion coefficients," Industrial \& Engineering Chemistry Research, vol. 58, no. 5, pp. 18-27, 1966.

[26] J. Kestin, K. Knierim, E. A. Mason, B. Najafi, S. T. Ro, and M. Waldman, "equilibrium and transport properties of the noble gases and their mixtures at low density," Journal of Physical and Chemical Reference Data, vol. 13, no. 1, 75 pages, 1984.

[27] A. Arteconi, G. Di Nicola, G. Santori, and R. Stryjek, "Second virial coefficients for dimethyl ether," Journal of Chemical \& Engineering Data, vol. 54, no. 6, pp. 1840-1843, 2009.

[28] A. A. Richcardo and M. N. da Ponte, "Second virial coefficients of mixtures of Xenon and lower hydrocarbons. 1. Experimental apparatus and results for $\mathrm{Xe}+\mathrm{C}_{2} \mathrm{H}_{6}$," The Journal of Physical Chemistry, vol. 100, no. 48, pp. 18839-18843, 1996.

[29] D. R. Lide, CRC Handbook of Chemistry and Physics, CRC Press, New York, NY, USA, 90th edition, 2010.

[30] J. Kestin and J. Yata, "Viscosity and diffusion coefficient of six binary mixtures," The Journal of Chemical Physics, vol. 49, no. 11, pp. 4780-4791, 1968.

[31] A. S. Kalelkar and J. Kestin, "Viscosity of He-Ar and $\mathrm{He}-\mathrm{Kr}$ binary gaseous mixtures in the temperature range $25-720^{\circ} \mathrm{C}$," The Journal of Chemical Physics, vol. 52, no. 8, pp. 4248-4261, 1970.

[32] J. Kestin, E. Paykoç, and J. V. Sengers, "On the density expansion for viscosity in gases,” Physica, vol. 54, no. 1, pp. 1-19, 1971.

[33] J. Kestin, S. T. Ro, and Ber. Bunsenges, "The viscosity of nine binary and two ternary mixtures of gases at low density," Berichte der Bunsengesellschaft für Physikalische Chemie, vol. 20, no. 1, pp. 20-24, 1974.

[34] J. Kestin, S. T. Ro, and W. A. Wakeham, "Viscosity of the binary gaseous mixture Helium-nitrogen," The Journal of Chemical Physics, vol. 56, no. 8, pp. 4032-4036, 1972.

[35] J. Kestin, S. T. Ro, and W. A. Wakeham, "Viscosity of the noble gases in the temperature range $25-700^{\circ} \mathrm{C}$," Journal of Chemical Physics, vol. 56, no. 8, 6 pages, 1972. 
[36] J. Kestin, S. T. Ro, and W. A. Wakeham, "Viscosity of the binary gaseous mixtures $\mathrm{He}-\mathrm{Ne}$ and $\mathrm{Ne}-\mathrm{N}_{2}$ in the Temperature Range 25-700 C', Journal of Chemical Physics, vol. 56, no. 12, 6 pages, 1972.

[37] J. Kestin, H. E. Kalifa, S. T. Ro, and W. A. Wakeham, "The viscosity and diffusion coefficients of eighteen binary gaseous systems," Physica A, vol. 88, no. 2, pp. 242-260, 1977.

[38] J. Kestin, H. E. Khalifa, and W. A. Wakeham, "The viscosity and diffusion coefficients of the binary mixtures of Xenon with the other noble gases," Physica A, vol. 90, no. 2, pp. 215-228, 1978.

[39] J. Kestin and W. A. Wakeham, "The viscosity and diffusion coefficient of binary mixtures of nitrous oxide with $\mathrm{He}, \mathrm{Ne}$ and CO," Berichte der Bunsengesellschaft für Physikalische Chemie, vol. 87, no. 4, pp. 309-311, 1983.

[40] M. Trauntz and K. F. Kipphan, "Die Reibung, Wärmeleitung und diffusion in gasmischungen. IV. Die reibung binärer und ternärer edelgasgemische," Annalen der Physik, vol. 394, no. 6, p. 743, 1929.

[41] M. Trauntz and R. Zink, "Die Reibung, Wärmeleitung und diffusion in gasmischungen XII. Gasreibung bei höheren temperaturen," Annalen der Physik, vol. 399, no. 4, p. 427, 1930.

[42] M. Trauntz and H. Zimmermann, "Die Reibung, Wärmeleitung und diffusion von gasmischungen XXX. Die innere reibung bei tiefen temperaturen von wasserstoff, helium und neon und binären gemischen davon bis 90,0 abs. herab," Annalen der Physik, vol. 414, no. 2, p. 189, 1935.

[43] J. Kestin and W. Leidenfrost, "The viscosity of Helium," Physica, vol. 25, no. 1-6, pp. 537-555, 1959.

[44] K. Kestin and W. Leidenfrost, Thermal and Transport Properties of Gases and Liquids, McGraw-Hill, New York, NY, USA, 1959.

[45] E. Thornton, "Viscosity and thermal conductivity of binary gas mixtures: Xenon-Krypton, Xenon-Argon, Xenon-Neon and Xenon-Helium," Proceedings of the Physical Society, vol. 76, no. 1, p. 104, 1960.

[46] E. Thornton, "Viscosity and thermal conductivity of binary gas mixtures: Krypton-Argon, Krypton-Neon, and KryptonHelium," Proceedings of the Physical Society, vol. 77, no. 6, p. 1166, 1961.

[47] E. Thornton and W. A. D. Baker, "Viscosity and thermal conductivity of binary gas mixtures: Argon-Neon, Argon-Helium, and Neon-Helium," Proceedings of the Physical Society, vol. 80, no. 5 , p. 1171, 1962.

[48] H. Iwasaki and J. Kestin, "The viscosity of Argon-Helium mixtures," Physica, vol. 29, no. 12, pp. 1345-1372, 1963.

[49] A. B. Rakshit and C. S. Roy, "Viscosity and polar-nonpolar interactions in mixtures of inert gases with ammonia," Physica, vol. 78, no. 1, pp. 153-164, 1974.

[50] A. O. Rietveld, A. Van Itterbeek, and G. J. Van Den Berg, "Measurements on the viscosity of mixtures of Helium and Argon," Physica, vol. 19, no. 1-12, pp. 517-524, 1953.

[51] A. O. Rietveld, A. Van Itterbeek, and C. A. Velds, "Viscosity of binary mixtures of hydrogen isotopes and mixtures of $\mathrm{He}$ and Ne," Physica, vol. 25, no. 1-6, pp. 205-216, 1959.

[52] A. E. Schuil, "A note on the viscosity of gases and molecular mean free path," Philosophical Magazine Series 7, vol. 28, no. 191, p. $679,1939$.

[53] J. Kestin and A. Nagashima, "Viscosity of Neon-Helium and Neon-Argon mixtures at $20^{\circ}$ and $30^{\circ} \mathrm{C}$," The Journal of Chemical Physics, vol. 40, no. 12, pp. 3648-3654, 1964.

[54] J. Kestin, Y. Kobayashi, and R. T. Wood, "The viscosity of four binary, gaseous mixtures at $20^{\circ}$ and $30^{\circ} \mathrm{C}$," Physica, vol. 32, no. 6, pp. 1065-1089, 1966.
[55] H. Landolt and R. Börnstein, Landolt-Börnstein Physikalischchemische Tabellen, Springer, Berlin, Germany, 1923.

[56] N. B. Vargaftik, Tables on the Thermophysical Properties of Liquids and Gases, Hemisphere, Washington, DC, USA, 2nd edition, 1975.

[57] K. Stephan and T. Heckenberger, Thermal Conductivity and Viscosity Data of Fluid Mixtures, vol. 10 of Chemistry Data Series, Dechema, Frankfurt, Germany, 1988.

[58] E. Bich, J. Millat, and E. Vogel, "The viscosity and thermal conductivity of pure monatomic gases from their normal boiling point up to $5000 \mathrm{~K}$ in the limit of zero density and at $0.101325 \mathrm{MPa}$," Journal of Physical and Chemical Reference Data, vol. 19, no. 6, article 1289, 17 pages, 1990.

[59] W. A. Wakeham, A. Nagashima, and J. V. Sengers, Measurement of the Transport Properties of Fluids, IUPAC Chemical Data Series no. 37, Blackwell Scientific, London, UK, 1991.

[60] A. Bejan and A. D. Kraus, Heat Transfer Handbook, chapter 2, John Wiley \& Sons, New York, NY, USA, 2003.

[61] G. C. Maitland and E. B. Smith, "Critical reassessment of viscosities of 11 common gases," Journal of Chemical \& Engineering Data, vol. 17, no. 2, pp. 150-156, 1972.

[62] G. F. C. Rogers and Y. R. Mayhew, Thermodynamic and Transport Properties of Fluids, Basil Blackwell, Oxford, UK, 3rd edition, 1981.

[63] IAEA, Thermophysical Properties of Materials for Nuuclear Engineering: A Tutorial and Collection of Data, IAEA, Vienna, 2008.

[64] J. Kestin, W. Wakeham, and K. Watanabe, "Viscosity, thermal conductivity, and diffusion coefficient of Ar-Ne and Ar-Kr gaseous mixtures in the temperature range $25-700^{\circ} \mathrm{C}$," The Journal of Chemical Physics, vol. 53, no. 10, pp. 3773-3780, 1970.

[65] J. Kestin, S. T. Ro, and W. A. Wakeham, "Viscosity of the binary gaseous mixture Neon-Krypton," Journal of Chemical Physics, vol. 56, no. 8, article 4086, 6 pages, 1972.

[66] A. O. Rankine, "On the variation with temperature of the viscosities of the gases of the Argon group," Zeitschrift für Physik, vol. 11, p. 497608, pp. 745-762, 1910.

[67] A. G. Clarke and E. B. Smith, "Low-temperature viscosities of argon, krypton, and xenon," The Journal of Chemical Physics, vol. 48, no. 9, pp. 3988-3991, 1968.

[68] R. A. Dawe and E. B. Smith, "Viscosities of the inert gases at high temperatures," The Journal of Chemical Physics, vol. 52, no. 2, pp. 693-703, 1970.

[69] R. S. Edwards, "The effect of temperature on the viscosity of Neon," Proceedings of the Royal Society A, vol. 119, no. 783, pp. 578-590, 1928.

[70] A. van Itterbeek and O. van Paemel, "Viscosity of liquid deuterium," Physica, vol. 7, no. 3, p. 208, 1940.

[71] R. Wobser and F. Müller, "Die innere reibung von gasen und dämpfen und ihre messung im Höppler-viskosimeter," KolloidBeihefte, vol. 52, no. 6-7, pp. 165-276, 1941.

[72] H. Johnston and E. R. Grilly, "Viscosities of carbon monoxide, Helium, Neon, and Argon between $80^{\circ}$ and $300^{\circ} \mathrm{K}$. coefficients of viscosity," The Journal of Physical Chemistry, vol. 46, no. 8, pp. 948-963, 1942.

[73] J. Kestin and W. Leidenfrost, "An absolute determination of the viscosity of eleven gases over a range of pressures," Physica, vol. 25, no. 7-12, pp. 1033-1062, 1959.

[74] G. P. Flynn, R. V. Hanks, N. A. Lemaire, and J. F. Ross, "Viscosity of Nitrogen, Helium, Neon, and Argon from $-78.5^{\circ}$ to $100^{\circ} \mathrm{C}$ 
below 200 Atmospheres," Journal of Chemical Physics, vol. 38, no. 1, article 154, 9 pages, 1963.

[75] J. Kestin and J. H. Whitelaw, "A relative determination of the viscosity of several gases by the oscillating disk method," Physica, vol. 29, no. 4, pp. 335-356, 1963.

[76] N. J. Trappeniers, A. Botzen, H. R. Van Den Berg, and J. Van Oosten, "The viscosity of Neon between $25^{\circ} \mathrm{C}$ and $75^{\circ} \mathrm{C}$ at pressures up to 1800 atmospheres. Corresponding states for the viscosity of the noble gases up to high densities," Physica, vol. 30, no. 5, pp. 985-996, 1964.

[77] A. O. Rietveld and A. van Itterbeek, "Measurements on the viscosity of Ne-A mixtures between 300 and $70^{\circ} \mathrm{K}$," Physica, vol. 22, no. 6-12, pp. 785-790, 1956.

[78] M. Trautz and H. E. Binkele, "Die Reibung, Wärmeleitung und diffusion in gasmischungen. VIII. die reibung des $\mathrm{H}_{2}, \mathrm{He}, \mathrm{Ne}$, Ar und ihrer binären gemische," Annalen der Physik, vol. 397, no. 5, pp. 561-580, 1930.

[79] B. A. Younglove and H. J. M. Hanley, "The viscosity and thermal conductivity coefficients of gaseous and liquid Argon," Journal of Physical and Chemical Reference Data, vol. 5, no. 4, article 1323, 15 pages, 1986.

[80] I. A. Barr, G. P. Matthews, E. B. Smith, and A. R. Tindell, "Intermolecular forces and the gaseous viscosities of ArgonXenon mixtures," Journal of Physical Chemistry, vol. 85, no. 22, pp. 3342-3347, 1981.

[81] J. M. Hellemans, J. Kestin, and S. T. Ro, "Viscosity of the binary gaseous mixtures of nitrogen with Argon and Krypton," The Journal of Chemical Physics, vol. 57, no. 9, pp. 4038-4042, 1972.

[82] J. Kestin and S. T. Ro, "The viscosity and diffusion coefficients of binary mixtures of nitrous Oxide with $\mathrm{Ar}, \mathrm{N}_{2}$, and $\mathrm{CO}_{2}$," Berichte der Bunsengesellschaft für Physikalische Chemie, vol. 86, no. 10, pp. 948-950, 1982.

[83] J. Kestin and W. A. Wakaham, "The viscosity of three polar gases," Berichte der Bunsengesellschaft für Physikalische Chemie, vol. 83, no. 6, pp. 573-576, 1979.

[84] A. van Itterbeek and O. van Paemel, "Measurements on the viscosity of Argon gas at room temperature and between $9^{\circ}$ and $55^{\circ}$ K," Physica, vol. 5, no. 10, pp. 1009-1012, 1938.

[85] P. Gray and A. O. S. Maczek, "The thermal conductivities, viscositites, and diffusion coefficients of mixtures, containing two polar gases," in Proceedings of the 4th Symposium on Thermophysical Properties, pp. 380-391, ASME, New York, NY, USA, 1968.

[86] C. F. Bonilla, S. J. Wang, and H. Weiner, "The viscosity of steam, heavy-water vapor, and Argon at atmospheric pressure up to high temperatures," Transactions of the American Society of Mechanical Engineers, vol. 78, pp. 1285-1289.

[87] H. Iwasaki, J. Kestin, and A. Nagashima, "Viscosity of Argonammonia mixtures," The Journal of Chemical Physics, vol. 40, no. 10, pp. 2988-2995, 1964.

[88] J. Hilsenrath, Tables of Thermal Properties of Gases, vol. 564 of National Bureau of Standards Circular, US Government Printing Office, Washington, DC, USA, 1955.

[89] J. M. Hellemans, J. Kestin, and S. T. Ro, "The viscosity of oxygen and of some of its mixtures with other gases," Physica, vol. 65, no. 2, pp. 362-375, 1973.

[90] E. F. May, R. F. Berg, and M. R. Moldover, "Reference viscosities of $\mathrm{H}_{2}, \mathrm{CH}_{4}$, Ar, and Xe at low densities," International Journal of Thermophysics, vol. 28, no. 4, pp. 1085-1110, 2007.

[91] V. Vasilesco, "Recherches expérimentales sur la viscosité des gaz aux températures élevées," Annales de Physique, vol. 20, pp. 292$334,1945$.
[92] H. J. M. Hanley, "the viscosity and thermal conductivity coefficients of dilute Argon, Krypton, and Xenon," Journal of Physical and Chemical Reference Data, vol. 3, no. 3, article 619, 24 pages, 1973.

[93] H. J. M. Hanley, R. D. McCarty, and W. M. Haynes J, “The viscosity and thermal conductivity coefficients for dense gaseous and liquid Argon, Krypton, Xenon, Nitrogen, and Oxygen," Journal of Physical and Chemical Reference Data, vol. 3, no. 4, article 979, 39 pages, 1974.

[94] X. Wang, J. Wu, and Z. Liu, "Viscosity of gaseous HFC245fa," Journal of Chemical \& Engineering Data, vol. 55, no. 1, pp. 496499, 2010.

[95] R. Kiyama and T. Makita, "The viscosity of carbon dioxide, ammonia, acetylene, Argon and oxygen under high pressures," Review of Physical Chemistry of Japan, vol. 22, pp. 49-58, 1952.

[96] T. Makita, "The viscosity at pressures to $80 \mathrm{o} \mathrm{kg} / \mathrm{cm}^{2}$ up of Argon, nitrogen and air," Review of Physical Chemistry of Japan, vol. 27, no. 1, pp. 16-21, 1957.

[97] M. Hongo, "Viscosity of Argon and of Argon-ammonia mixtures under pressures," The Review of Physical Chemistry of Japan, vol. 48, no. 2, pp. 63-71, 1979.

[98] J. Kestin, H. E. Khalifa, and W. A. Wakeham, "The viscosity of gaseous mixtures containing Krypton," The Journal of Chemical Physics, vol. 67, no. 9, pp. 4254-4259, 1977.

[99] M. Trautz, "Die reibung, Wärmeleitung und diffusion in Gasmischungen XXI. Absoluter $\eta$-Wirkungsquerschnitt, molekulartheoretische bedeutung der kritischen temperatur und berechung kritischer drucke aus $\eta$," Annalen Der Physik, vol. 407, no. 2, p. 198, 1932.

[100] J. M. Gandhi and S. C. Saxena, "Thermal conductivity of binary and ternary mixtures of Helium, Neon and Xenon," Molecular Physics, vol. 12, no. 1, p. 57, 1967.

[101] S. C. Saxena, M. P. Saksena, R. S. Gambhir, and J. M. Gandhi, "The thermal conductivity of nonpolar polyatomic gas mixtures," Physica, vol. 31, no. 3, pp. 333-341, 1965.

[102] H. Ziebland, "commission on physicochemical measurements and standards," Pure \& Applied Chemistry, vol. 53, no. 10, pp. 1863-1877, 1981.

[103] R. W. Powell, C. Y. Ho, and P. E. Liley, “Thermal conductivity of seleted materials," National Standard Reference Data Series NBS 8, US Department of Commerce, 1966.

[104] S. C. Saxena, "Transport properties of gases and gaseous mixtures at high temperatures," High Temperature Science, vol. 3, pp. 168-188, 1971.

[105] S. C. Saxena, S. Mathur, and G. P. Gupta, "The thermal conductivity data of some binary gas mixtures involving nonpolar polyatomic gases," Defence Science Journal, vol. 16, supplement, pp. 99-112, 1966.

[106] N. B. Vargaftik, L. P. Filippon, A. A. Tarzimanov, and E. E. Totskii, Handbook of Thermal Conductivity of Liquids and Gaseous, CRC Press, Boca Raton, Fla, USA, 1994.

[107] W. Van Dael and H. Cauwenbergh, "Measurements of the thermal conductivity of gases. II. Data for binary mixtures of He, Ne and Ar," Physica, vol. 40, no. 2, pp. 173-181, 1968.

[108] W. G. Kannuluik and E. H. Carman, "The thermal conductivity of rare gases," Proceedings of the Physical Society B, vol. 65, no. 9, p. 701, 1952.

[109] R. S. Gambhir and S. C. Saxena, "Thermal conductivity of binary and ternary mixtures of Krypton, Argon and Helium," Molecular Physics, vol. 11, no. 3, pp. 233-241, 1966. 
[110] E. A. Mason and H. Von Ubisch, "Thermal conductivities of rare gas mixtures," Physics of Fluids, vol. 3, no. 3, pp. 355-361, 1960.

[111] S. C. Saxena, "Thermal conductivity of binary and ternary mixtures of helium, argon and xenon," Indian Journal of Physics, vol. 31, pp. 597-606, 1957.

[112] H. von Ubisch, "The thermal conductivities of mixtures of rare gases at $29^{\circ}$ and 520", Arkiv för Fysik, vol. 16, pp. 93-100, 1959.

[113] S. Mathur, P. K. Tondon, and S. C. Saxena, "Thermal conductivity of binary, ternary and quaternary mixtures of rare gases," Molecular Physics, vol. 12, no. 6, pp. 569-579, 1967.

[114] C. Y. Ho, R. W. Powell, and P. E. Liley J, “Thermal conductivity of the elements," Journal of Physical and Chemical Reference Data, vol. 1, no. 2, article 279, 143 pages, 1972.

[115] B. N. Srivastava and S. C. Saxena, "Thermal conductivity of binary and ternary rare gas mixtures," Proceedings of the Physical Society B, vol. 70, no. 4, p. 369, 1957.

[116] K. Schäfer, "Transport Phenomena in the temperature range up to 1100 degrees," Dechema Monograph, vol. 32, pp. 61-73, 1959.

[117] K. C. Hansen, L. H. Tsao, T. M. Aminabhavi, and C. L. Yaws, "Gaseous thermal conductivity of hydrogen chloride, hydrogen bromide, boron trichloride, and boron trifluoride in the temperature range from 55 to $380^{\circ} \mathrm{C}$," Journal of Chemical and Engineering Data, vol. 40, no. 1, pp. 18-20, 1995.

[118] R. S. Gambhir and S. C. Saxena, "Thermal conductivity of the gas mixtures: Ar- $\mathrm{D}_{2}, \mathrm{Kr}-\mathrm{D}_{2}$ and Ar-Kr- $\mathrm{D}_{2}$," Physica, vol. 32, no. 11-12, pp. 2037-2043, 1966.

[119] L. Sun and J. E. S. Venart, "Thermal conductivity, thermal diffusivity, and heat capacity of gaseous Argon and nitrogen," International Journal of Thermophysics, vol. 26, no. 2, pp. 325372, 2005.

[120] L. Sun, J. E. S. Venart, and R. C. Prasad, "The thermal conductivity, thermal diffusivity, and heat capacity of gaseous Argon," International Journal of Thermophysics, vol. 23, pp. 357389, 2002.

[121] C. J. Zwakhals and K. W. Reus, "Corrections to the Smoluchowski equation in the presence of hydrodynamic interactions," Physica C, vol. 100, no. 2, pp. 251-265, 1980.

[122] W. Groth and E. Sußner, "Selbstdiffusionsmessungen III. Der selbstdiffusionskoeffizient des neon (self-diffusion measurments III. The coefficient of self diffusion of neon)," Zeitschrift für Physikalische Chemie, vol. 193, pp. 296-300, 1944.

[123] W. Groth and P. Harteck, "Die selbstdiffusion des Xenons und des Kryptons," Zeitschrift für Elektrochemie, vol. 47, no. 2, pp. 167-172, 1941.

[124] M. P. Saksena and S. C. Saxena, "Viscosity of multicomponent gas mixtures," Physica A, vol. 31, p. 18, 1965.

[125] H. Iwasaki and J. Kestin, "The viscosity of Argon-Helium mixtures," Physica, vol. 29, no. 12, pp. 1345-1372, 1963.

[126] D. J. Richardson, G. Mason, B. A. Buffham, K. Hellgardt, I. W. Cumming, and P. A. Russell, "Viscosity of binary mixtures of carbon monoxide and Helium," Journal of Chemical and Engineering Data, vol. 53, no. 1, pp. 303-306, 2008.

[127] S. C. Saxena and T. K. S. Narayanan, "Multicomponent viscosities of gaseous mixtures at high temperatures," Industrial \& Engineering Chemistry Fundamentals, vol. 1, no. 3, pp. 191-195, 1962.

[128] J. W. Buddenberg and C. R. Wilke, "Calculation of gas mixture viscosities," Industrial \& Engineering Chemistry Research, vol. 41, no. 7, pp. 1345-1347, 1949.

[129] J. Wachsmuth, "Über die wärmeleitung von gemischen zwischen argon und helium," Physikalische Zeitschrift, vol. 7, p. 235, 1908.
[130] E. A. Mason and T. R. Marrero, "Gaseous diffusion coefficients," Journal of Physical and Chemical Reference Data, vol. 1, no. 1, 116 pages, 1972.

[131] R. J. J. Van Heijningen, J. P. Harpe, and J. J. M. Beenakker, "Determination of the diffusion coefficients of binary mixtures of the noble gases as a function of temperature and concentration," Physica, vol. 38, no. 1, pp. 1-34, 1968.

[132] P. S. Arora, H. L. Robjohns, and P. J. Dunlop, "Use of accurate diffusion and second virial coefficients to determine $(\mathrm{m}, 6,8)$ potential parameters for nine binary noble gas systems," Physica $A$, vol. 95, no. 3, pp. 561-571, 1979.

[133] M. Trautz and W. Muller, "Die reibung, wärmeleitung und diffusion in gasmischungen III. Die korrektion der bisher mit der verdampfungsmethode gemessenen diffusionskonstanten," Annalen der Physik, vol. 414, no. 4, pp. 333-352, 1935.

[134] J. N. Holsen and M. R. Strunk, "Binary diffusion coefficients in nonpolar gases," Industrial and Engineering Chemistry Fundamentals, vol. 3, no. 2, pp. 143-146, 1964.

[135] S. L. Seager, L. R. Geertson, and J. C. Giddings, "Temperature dependence of gas and vapor diffusion coefficients," Journal of Chemical \& Engineering Data, vol. 8, no. 2, pp. 168-169, 1963.

[136] A. T. Hu and R. Kobayashi, "Measurements of gaseous diffusion coefficients for dilute and moderately dense gases by perturbation chromatography," Journal of Chemical \& Engineering Data, vol. 15, no. 2, pp. 326-335, 1970.

[137] A. P. Malinauskas, "Gaseous diffusion. The systems He-Ar, Ar$\mathrm{Xe}$, and He-Xe," Journal of Chemical Physics, vol. 42, no. 1, article 157, 4 pages, 1965.

[138] R. A. Strehlow, "The temperature dependence of the mutual diffusion coefficient for four gaseous systems," Journal of Chemical \& Engineering Data, vol. 21, no. 12, article 2101, 6 pages, 1953.

[139] R. E. Walker and A. A. Westenberg, "Molecular diffusion studies in gases at high temperature. III. results and interpretation of the He-a system," Journal of Chemical Physics, vol. 31, no. 519, 4 pages, 1959.

[140] J. C. Giddings and S. L. Seager, "Method for the rapid determination of diffusion coefficients. theory and application," Industrial and Engineering Chemistry Fundamentals, vol. 1, no. 4, pp. 277-283, 1962.

[141] S. C. Sexena and E. A. Mason, "Thermal diffusion and the approach to the steady state in gases: II," Molecular Physics, vol. 2, no. 4, p. 379, 1959.

[142] E. N. Fuller, P. D. Schettler, and J. C. Giddings, "New method for prediction of binary gas-phase diffusion coefficients," Industrial \& Engineering Chemistry, vol. 58, no. 5, pp. 18-27, 1966.

[143] M. Keil, L. Danielson, and P. J. Dunlop, "On obtaining interatomic potentials from multiproperty fits to experimental data," Journal of Chemical Physics, vol. 84, no. 14, article 296, 14 pages, 1991.

[144] A. P. Malinauskas, "Gaseous Diffusion. The Systems He-Ar, Ar$\mathrm{Xe}$, and He-Xe," Journal of Chemical Physics, vol. 42, no. 1, article 156, 4 pages, 1964.

[145] K. Schafer and K. Schuhman Z, "Zwischenmolekulare kräfte und temperaturabhängigkeit von diffusion und selbstdiffusion in Edelgasen," Zeitschrift für Elektrochemie, vol. 61, no. 2, pp. 246-252, 1957.

[146] B. N. Srivastava and K. P. Srivastava, "Mutual diffusion of pairs of rare gases at different temperatures," Journal of Chemical Physics, vol. 30, no. 984, article 984, 7 pages, 1959.

[147] W. Sutherland, "The viscosity of gases and molecular force," Philosophical Magazine, vol. 36, pp. 507-631, 1893. 
[148] W. Sutherland, "The attraction of unlike molecules.-I. The diffusion of gases," Philosophical Magazine, vol. 38, no. 230, pp. $1-19,1894$.

[149] V. P. S. Main and S. C. Saxena, "measurement of the concentration diffusion coefficient for Ne-Ar, Ne-Xe, Ne- $\mathrm{H}_{2}, \mathrm{Xe}-\mathrm{H}_{2}$, $\mathrm{H}_{2}-\mathrm{N}_{2}$ and $\mathrm{H}_{2}-\mathrm{O}_{2}$ gas systems," Applied Scientific Research, vol. 23, pp. 121-133, 1971.

[150] A. E. Humphreys and E. A. Mason, "Intermolecular forces: thermal diffusion and diffusion in Ar-Kr," Physics of Fluids, vol. 13, no. 1, article 65, 6 pages, 1970.

[151] P. J. Dunlop and C. M. Bignell, "Diffusion and thermal diffusion in binary mixtures of methane with noble gases and of Argon with Krypton," Physica A, vol. 145, no. 3, pp. 584-596, 1987.

[152] L. Andrussow and T. F. Schatzki, "Diffusion coefficients of the systems Xe-Xe and A-Xe," Journal of Chemical Physics, vol. 27, no. 5, article 1049, 6 pages, 1957.

[153] E. A. Mason and T. R. Marrero, "The diffusion of atoms and molecules," Advances in Atomic and Molecular Physics, vol. 6, pp. 155-232, 1970. 

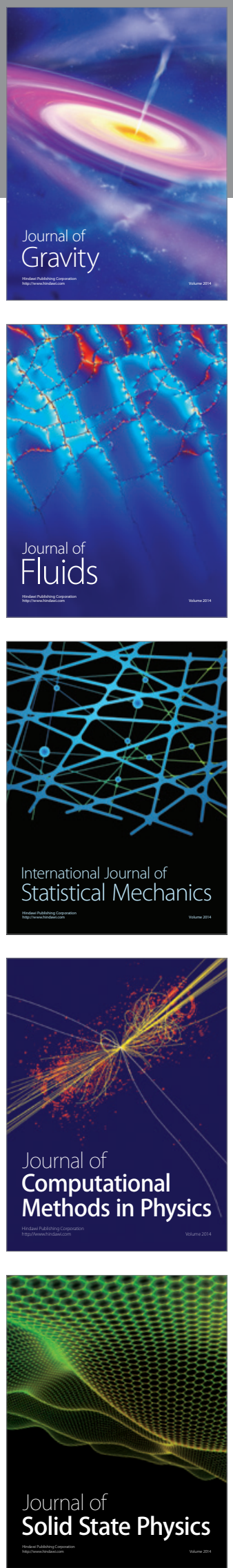

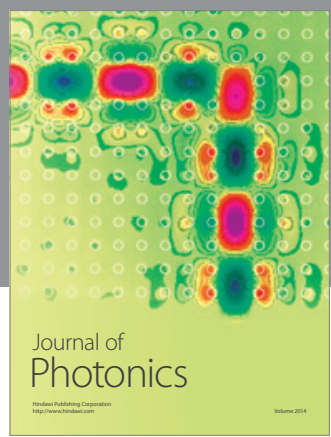

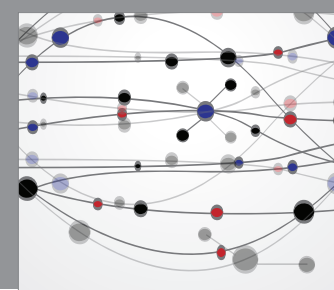

The Scientific World Journal

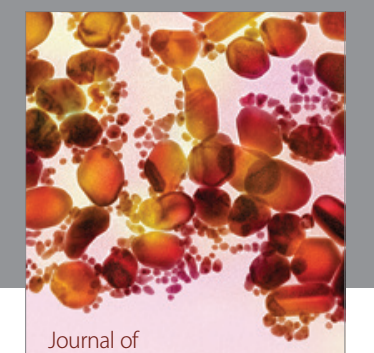

Soft Matter
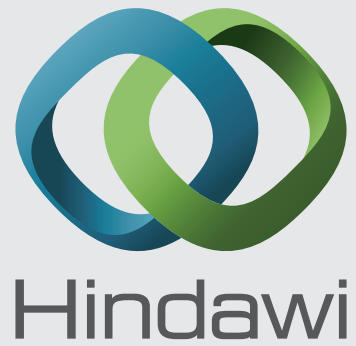

Submit your manuscripts at

http://www.hindawi.com
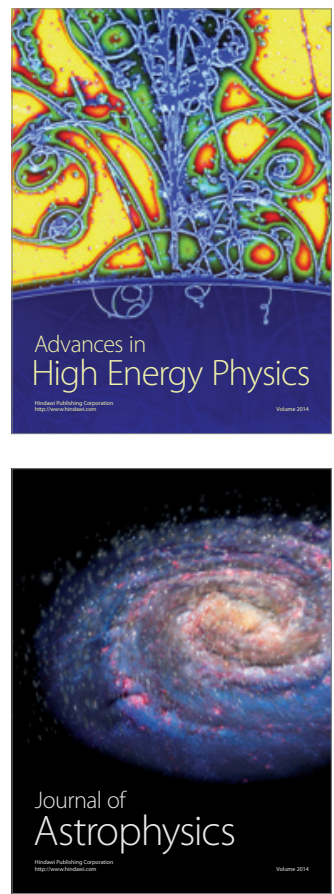
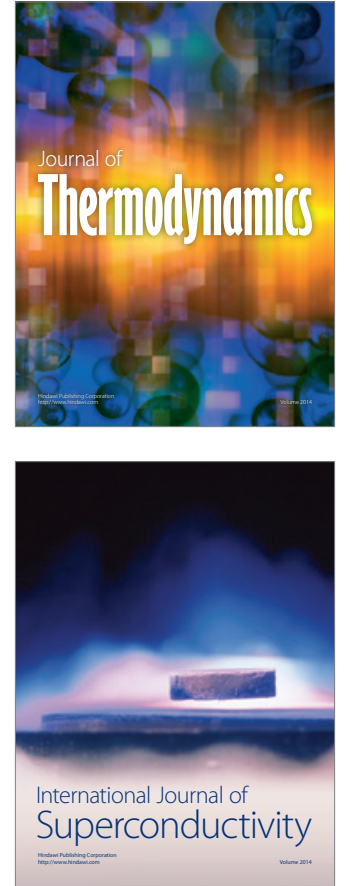
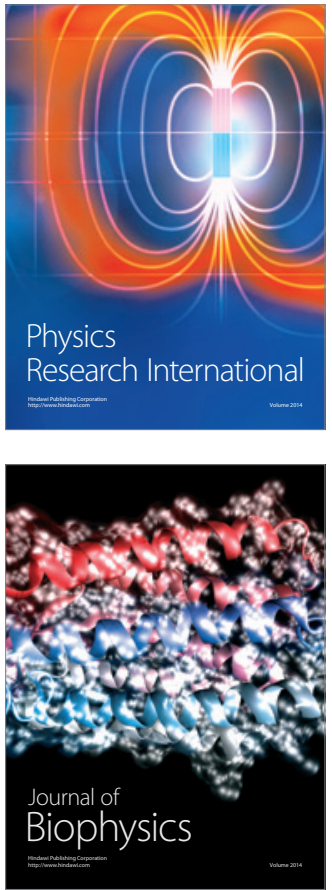
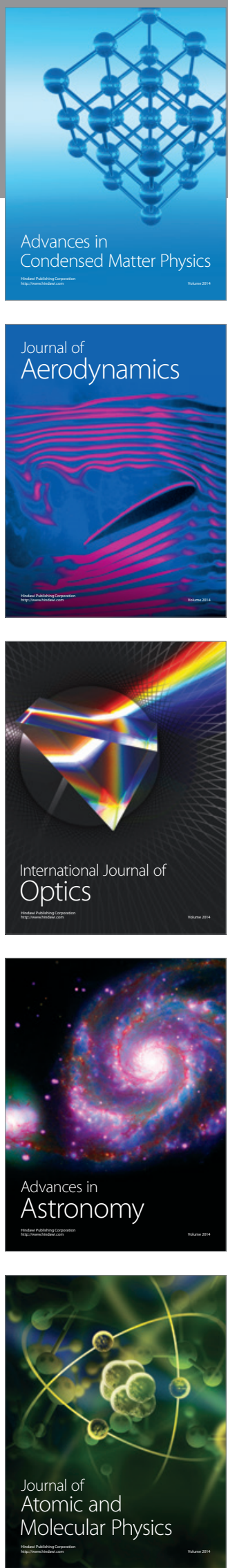